A Scaled Final Focus Experiment for Heavy Ion Fusion

by

Stephan Alexander MacLaren

B.S. (United States Naval Academy) 1990

M.A. (University of California, Berkeley) 1997

A dissertation submitted in partial satisfaction of the

requirements for the degree of

Doctor of Philosophy

in

Physics

in the

GRADUATE DIVISION

of the

UNIVERSITY OF CALIFORNIA, BERKELEY

Committee in charge:

Professor Joel Fajans, Co-chair

Dr. Peter Seidl, Co-chair

Professor Per Peterson

Professor Jonathan Wurtele

Fall 2000 


\section{A Scaled Final Focus Experiment for Heavy Ion Fusion}

Copyright (C) 2000

By

Stephan Alexander MacLaren

The U.S. Department of Energy has the right to use this document for any purpose whatsoever including the right to reproduce all of any part thereof. 


\begin{abstract}
A Scaled Final Focus Experiment for Heavy Ion Fusion*

by

Stephan Alexander MacLaren

Doctor of Philosophy in Physics

University of California, Berkeley

Professor Joel Fajans, Co-chair

Dr. Peter Seidl, Co-chair
\end{abstract}

A one-tenth dimensionally scaled version of a final focus sub-system design for a heavy ion fusion driver is built and tested. By properly scaling the physics parameters that relate particle energy and mass, beam current, beam emittance, and focusing field, the transverse dynamics of a driver scale final focus are replicated in a small laboratory beam. The experiment uses a $95 \mu \mathrm{A}$ beam of $160 \mathrm{keV} \mathrm{Cs}^{+}$ions to study the dynamics as the beam is brought to a ballistic focus in a lattice of six quadrupole magnets. Diagnostic stations along the experiment track the evolution of the transverse phase space of the beam. The measured focal spot size is consistent with calculations and the report of the design on which the experiment is based.

By uniformly varying the strengths of the focusing fields in the lattice, the chromatic effect of a small energy deviation on the spot size can be reproduced. This is done for $\pm 1 \%$ and $\pm 2 \%$ shifts and the changes in the focus are measured. Additionally, a $400 \mu \mathrm{A}$ beam is propagated through the experiment and partially neutralized after the last magnet using electrons released from a hot tungsten filament. The increase in beam

${ }^{*}$ This work was performed under the auspices of the Office of Fusion Energy Science, U.S. Department of Energy by University of California Lawrence Berkeley National Laboratory under contract No.DE-AC03-765F00098. 
current allows for the observation of significant effects on both the size and shape of the focal spot when the electrons are added. 
For my wife, Paulette

whose love and support are the wind in my sails 


\section{Contents}

1 INTRODUCTION ............................................................................... 1

1.1 Inertial Confinement Fusion .......................................................................................................1

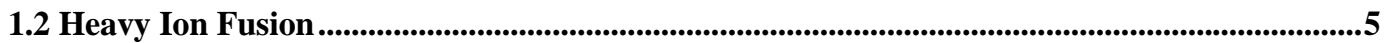

1.3 The Final Focus Sub-System and the HIBALL-II Design .......................................................10

1.4 Goals For This Work ..................................................................................................................................12

2 BEAM DYNAMICS AND SCALING ................................................ 15

2.1 The K-V Envelope Equation ...........................................................................................15

2.2 Scaling Relations..............................................................................................................................................20

2.3 Summary of Scaled Parameters ..............................................................................................................21

3 DESCRIPTION OF THE EXPERIMENT............................................ 23

3.1 Vacuum System and Beam Pulse Generator ......................................................................................23

3.2 Electrostatic Focusing Section .................................................................................................................26

3.3 Magnetic Focusing Section ..................................................................................................................29

3.4 Beam Diagnostics................................................................................................................................................32

4 DEVELOPMENT OF THE ION SOURCE ......................................... 39

4.1 Background ............................................................................................................................................................39

4.2 Mechanical Design .................................................................................................................................................40

4.3 Contact Ionization and Lifetime....................................................................................................................44

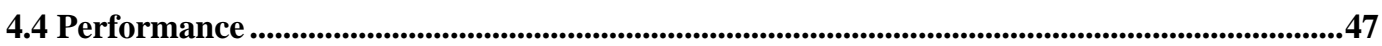

5 EXPERIMENTAL METHODS........................................................... 51

5.1 Data Acquisition System ...........................................................................................................................51

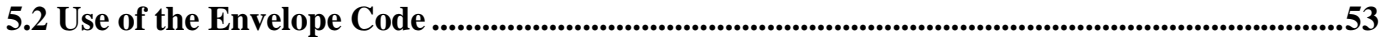

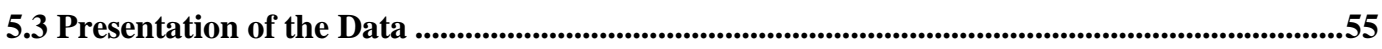




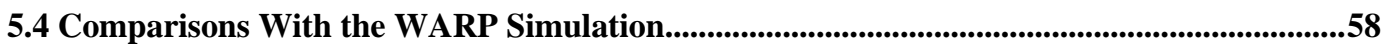

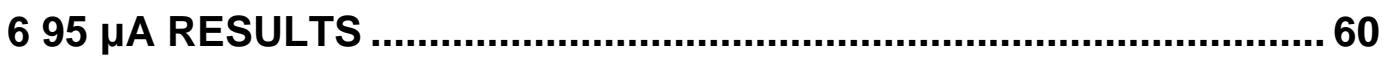

6.1 Data Before the Focus ..............................................................................................................................60

6.2 Predicted Spot Characteristics ....................................................................................................69

6.3 Spot Measurements .............................................................................................................................................73

6.4 Chromatic Aberration Studies......................................................................................................77

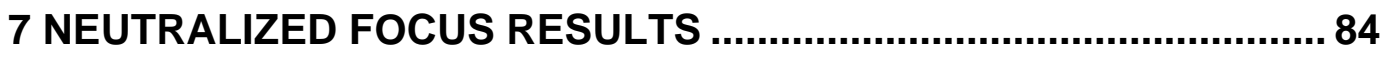

7.1 The Higher Current Solution.....................................................................................................................84

7.2 Measurement Of Neutralization with the Drift Tube..........................................................................91

7.3 Measured Spot and Inferred Neutralization.................................................................................93

7.4 Comparison With the LSP Model .....................................................................................97

8 ERROR ANALYSIS AND CONCLUSIONS...................................... 99

8.1 Linearity And Reproducability of the Slit-Cup Measurements...................................................99

8.2 Errors Associated with Two Slit Emittance Scans..............................................................................101

8.3 Error Associated with the Drift Tube Bias ..........................................................................104

8.4 Conclusions................................................................................................................................................................106

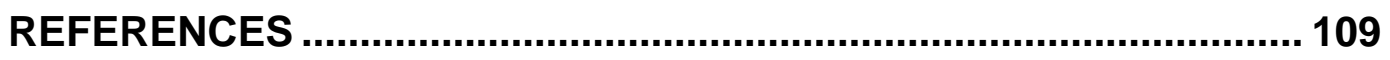

APPENDIX A: A CONDENSED HISTORY OF FINAL FOCUS STUDIES ..... 113

Appendix A References...........................................................................................................................................117 


\section{List of Figures}

Figure 1.1 Rate constants for several reactions as a function of temperature (Data from Feldbacher, 1987).

Figure 1.2: Simplified schematic of an IFE power plant ...................................... 4

Figure 1.3: Design for a high yield $(\mathrm{G}=130)$ indirect drive target ........................... 6

Figure 1.4: Ion range as a function of incident energy for various species .................... 7

Figure 1.5: Schematic of an accelerating cell in an induction accelerator...................... 9

Figure 3.1: Vacuum tanks housing the experiment. The electrostatic section is in the foreground, with the magnetic section in the background to the left...................... 24

Figure 3.2: Layout of the Scaled Final Focus Experiment ......................................... 25

Figure 3.3: Electrostatic transport section. The beam direction is from the background at left to the foreground at right. D1 and D2 diagnostics are visible at the top of the figure.

Figure 3.4: Magnetic Focusing Section Hardware ............................................... 29

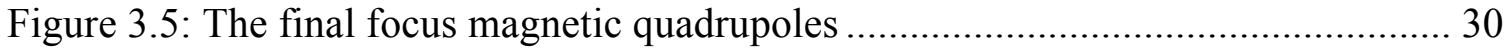

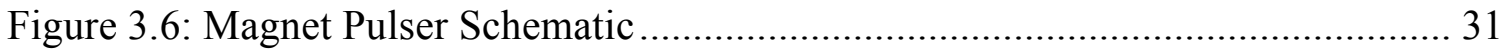

Figure 3.8: Simplified schematic of the slit-cup diagnostic circuitry.......................... 34

Figure 3.9: Measurements made using slit and slit-cup diagnostic probes.................... 35

Figure 3.10: Faraday cup (top) and drift tube (bottom) diagnostics in the drift region after the last magnet. To the left is the 3 -axis probe used for the focal spot................... 36

Figure 3.11: Comparison of the integrated signal from the drift tube to the signal from the co-located Faraday cup for an un-neutralized beam ....................................... 38

Figure 4.1: Cutaway view of the source enclosure and first electrostatic quadrupole .... 41

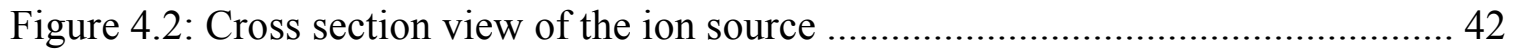

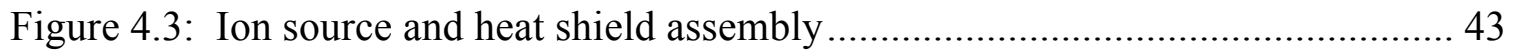

Figure 5.1: Phase program interface for sampling phase space and setting up the measurement boundary for an automated scan 
Figure 5.2: Plot of measured emittance versus percent of total signal for a phase space measurement at D4. The red line indicates where the emittance would be evaluated.

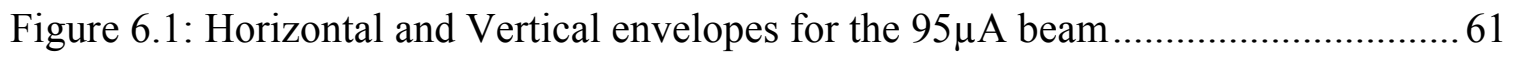

Figure 6.2: Transverse phase space at D1; measured and from PIC simulation .............. 62

Figure 6.3: Transverse phase space at D2; measured and from PIC simulation ............. 63

Figure 6.4: Vertical transverse phase space at D4; measured and from PIC simulation .. 65

Figure 6.5: Horizontal transverse phase space at D4; measured and from PIC simulation

Figure 6.6: Crossed slit measurements of beam current density at D1 and D4. For each measurement, the slit widths and the step size intervals are $1 \mathrm{~mm} . \ldots \ldots \ldots \ldots \ldots \ldots \ldots \ldots . . . . . . . . . . .68$

Figure 6.7: Graphical solution of equation 6-4 for the HIBALL-II design parameters .... 72

Figure 6.8: Graphical solution of equation 6-4 for the measured experimental parameters

Figure 6.9: Single slit scans in both transverse planes at the focal point. Widths of the raw data $\left(\mathrm{x}_{\mathrm{rms}}\right)$ and of the Gaussian fit $(\sigma)$ are indicated. ............................... 75

Figure 6.10: Percent of the beam current at the focus as a function of distance from the centroid of the distribution.

Figure 6.11: Percent of beam current at the focus as a function of radius assuming a two

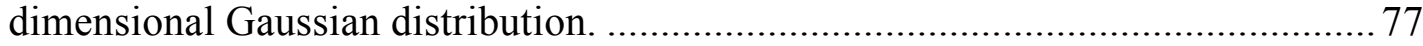

Figure 6.12 :Fractional increase in focal spot area for each of the energy shift trials. ..... 80

Figure 6.13: Percent of the beam current at the focus as a function of distance from the centroid of the distribution for each of the energy shift trials. Data from both the horizontal and vertical slit scans are shown on each plot.

Figure 6.14: The fraction of the current within a $0.4 \mathrm{~mm}$ radius is normalized to the onmomentum measurement and plotted as "Efficiency" for comparison to the HIBALL-II report.

Figure 7.1: Spot radius is plotted as a function of beam current leaving other parameters fixed, with the two points indicating the experimental values of the current. The red curve separates the emittance and space charge dominated regimes. 85

Figure 7.2 Horizontal and vertical envelopes calculated for the $400 \mu \mathrm{A}$ beam ...............86

Figure 7.3: Transverse phase space at D1; measured and from PIC simulation 87 
Figure 7.4 : Transverse phase space at D2; measured and from PIC simulation ............. 88

Figure 7.5 : Transverse phase space at D3; measured and from PIC simulation............ 89

Figure 7.6 Transverse phase space at D1; measured and from PIC simulation .............. 90

Figure 7.7: Single slit profiles of the $400 \mu \mathrm{A}$ focal spot. The hollow purple bars represent the un-neutralized focus, while the solid yellow bars represent the result of using the

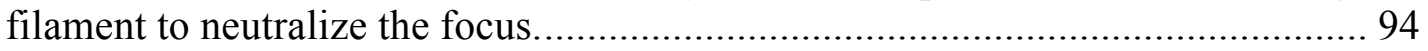

Figure 7.8: Time resolved slit measurement of the focal spot in the vertical plane........ 95

Figure 7.9: Calculated beam envelopes between D4 and the focus for both the neutralized

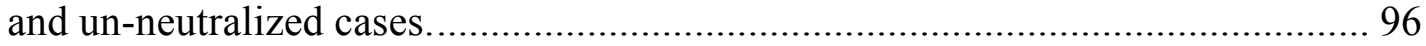

Figure 7.10 : The neutralized beam from the LSP run is plotted as it would be seen in a single slit scan. This data is compared to the measured spot.

Figure 8.1: Comparisons of a profile measurement made using the secondary electron signal (reverse bias) with the ion signal (forward bias). The ion signal has been scaled by a factor of 24.5

Figure 8.2: Illustration of a calculation of the expected measured velocity distribution from an intrinsic Gaussian distribution incident on a pair of slits of finite width...102

Figure 8.3: Spot size of the neutralized beam passing through the biased drift tube is plotted as a function of the negative bias voltage. 


\section{Acknowledgements}

That I was able to accomplish the work described in this dissertation is a testament to the unselfish contributions and support of many individuals. So many, in fact, that I must apologize in advance to those who are not, but should have been, included below. Here, then, is my heartfelt offering of gratitude and thanks, not quite commensurate to the kindness I have received.

I would like to begin by recognizing the late Dr. David Judd, who provided my introduction to the topic of heavy ion fusion. His teaching, guidance, and encouragement were invaluable during my first few years as a graduate student. I am grateful also to Professor Robert Jacobsen for his support and encouragement during that time.

As my mentors in the realm of experimental physics, I wish to thank Drs. Peter Seidl and Andris Faltens. A valuable resource on so many topics, Dr. Faltens championed the concept of a properly scaled experiment, and could always be sought out to provide a fresh viewpoint on a troublesome issue. As my research advisor, Dr. Seidl has been infinitely generous with his time and guidance, gently steering me towards the clearest method and the objective viewpoint. I would also like to express my appreciation to Dr. Joe Kwan, whose expertise as an experimentalist was solicited on a number of occasions. Finally, I would like to thank Professor Joel Fajans for serving as my faculty advisor from the University of California at Berkeley.

Always happy to discuss the physics of the experiment from the theoretical perspective were Drs. John Barnard and Christine Celata. I greatly appreciate their willingness to share their insight and experience when I came to them with questions. 
Dr. Enrique Henestroza contributed to the successful design of the ion source with his simulations of the diode.

The technical support and expertise made available to me in Building 58 was nothing short of outstanding. Mr. Ralph Hipple's superb management of the mechanical resources ensured that the experiment was never without the necessary material or manpower. I am deeply indebted to the skill and patience of Mr. William Ghiorso for turning a crude pencil sketch of the high-brightness ion source into reality, and for designing and building a focal spot diagnostic that performed many successful measurements. I greatly appreciate the work of Mr. Gary Ritchie in building the quadrupole magnets, and of Mr. Ron Beggs, who fabricated each of the other diagnostic devices and worked tirelessly to ensure the faultless operation of the vacuum system.

The electrical support staff, headed by Mr. William Strelo, is also due recognition for their efforts to keep the experimental systems in peak condition. I wish to thank Mr. Craig Rogers and Mr. Ed Romero for their invaluable work on equipment from the microvolt to the kilovolt range. Additionally, I would like to express my gratitude to $\mathrm{Mr}$. Gerald Stoker for his work on the electrical control system and safety interlocks, and Mr. David Beck for his engineering effort from the source to the focus.

I would like to show my appreciation to my officemates, Ms. Caron Jantzen and Mr. Michiel de Hoon, for their companionship as we struggled for long hours together forcing our will upon the most stubborn of software applications. I would also like to thank Michiel for his efforts in the simulation of the experiment, and his willingness to accommodate "just one more run" on numerous occasions without complaint. 
Finally, and most importantly, I wish to express my deep gratitude to my family for their encouragement and support of this endeavor. In particular, I would like to thank my mother, Janet Freeman, who inspired in me the drive to pursue learning to its limits, and my wife, Paulette Passanisi, who believed in my abilities even when I did not. Their caring and willingness to listen gave me the confidence to accomplish a lifelong goal.

Berkeley, California

STEPHAN A. MACLAREN

September, 2000 


\section{Introduction}

Inertial fusion of hydrogen isotopes is one of the premier alternatives to fossil fuels for the production of safe, clean energy. The concept has demonstrated a level of scientific feasibility that merits establishment of a development phase in which enabling technologies are identified and tested. As a driver for an inertial confinement fusion (ICF) power plant, heavy ion beams look extremely promising. Once these beams have been brought to high energies using currently available accelerator technology, it is critical that they deliver this energy to the fusion fuel, commonly known as the target. The subsystem of a heavy ion fusion (HIF) driver that accomplishes this task is often referred to as the final focus. This system must overcome various forces and cope with aberrations in order to provide the necessary power to an ICF target for an acceptable production of energy. The work presented here is concerned with studying a particular design for the final focus on a much smaller scale. Close attention is paid to reproducing the physics that are involved in producing the desired beam focus through careful scaling of the experimental parameters.

\subsection{Inertial Confinement Fusion}

Fusion is the process of combining light nuclei such as isotopes of hydrogen to form heavier ones. When they are joined, or fused, the nuclei release energy that is carried away by the product particles. In order to overcome the Coulomb forces between the nuclei, the fuel is heated to approximately $10^{8} \mathrm{~K}$. The velocity distributions of the species at this temperature provide for collisions that allow the nuclei to get close enough to fuse. 
Figure 1.1 gives the rate constants, $\langle\sigma v\rangle$, for several fusion reactions as a function of temperature. The reaction shown in the figure that combines deuterium and tritium has the largest peak rate constant, with a maximum between 20 and $100 \mathrm{keV}$. When these two isotopes of hydrogen fuse, they create an alpha particle and a neutron. The reaction produces $17.6 \mathrm{MeV}$ of energy, with the neutron carrying $14.1 \mathrm{MeV}$, and the alpha carrying the remaining $3.5 \mathrm{MeV}$. The neutron has a long range, and is therefore used to heat the coolant in the chamber, while the alpha particle has a very short range and provides heat to the fuel mass to sustain the reaction.

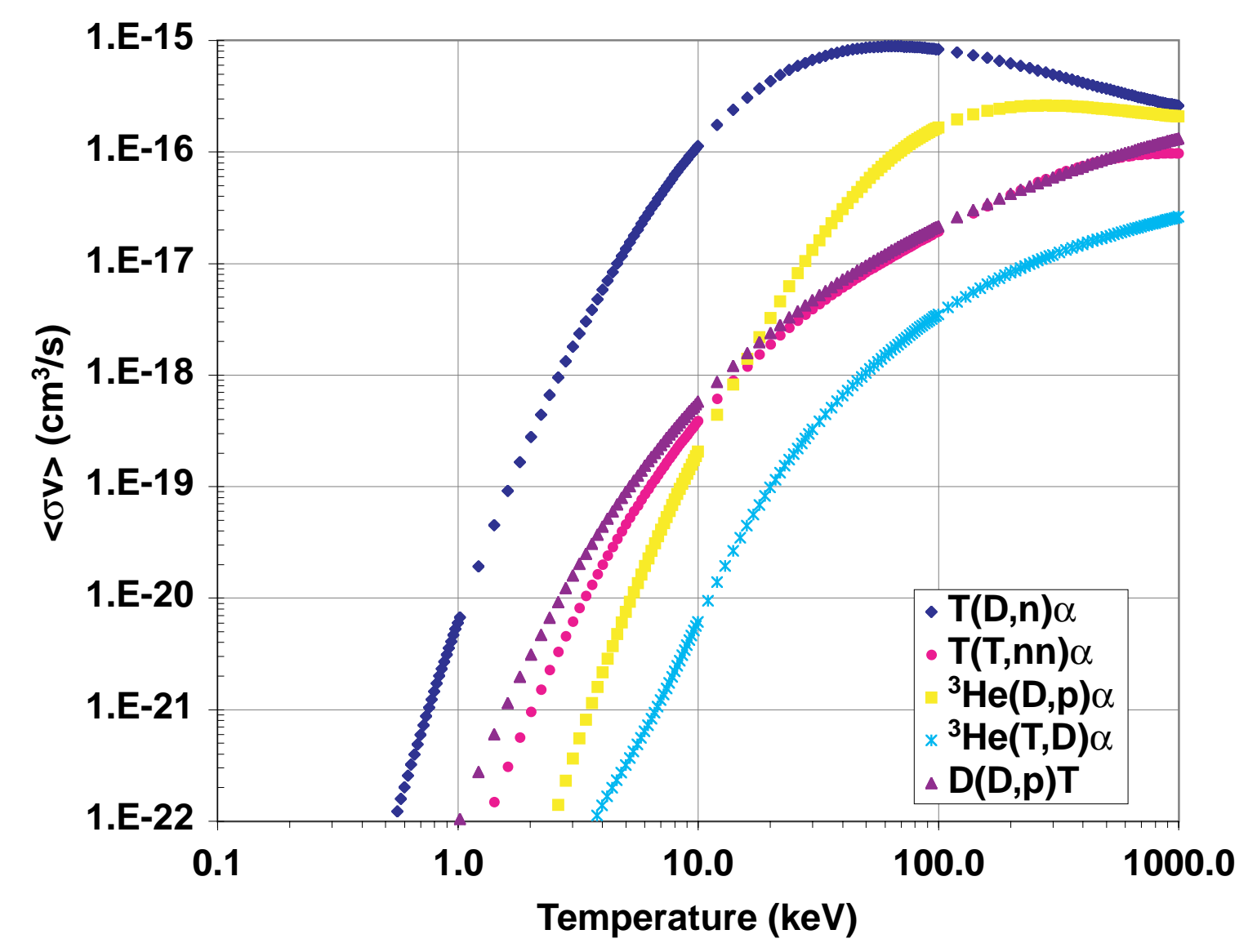

Figure 1.1 Rate constants for several reactions as a function of temperature (Data from Feldbacher, 1987).

To use this reaction to generate energy on a practical scale, not only must the fuel be at the appropriate temperature, but the material must be held together long enough for 
significant fraction to undergo the fusion reaction. If the fuel consists of an equal mixture of deuterium and tritium, $n_{D}=n_{T}=n / 2$, then

$\frac{d n_{R}}{d t}=\frac{n^{2}}{4}\langle\sigma v\rangle$

where $n_{R}$ is the number of fusion reactions per unit volume. A burn fraction $f$ can be defined by $n=n_{0}(1-f)$, where $n_{0}$ is the initial fuel density, and

$n_{R}=\frac{n_{0}-n}{2}$

Inserting Equation (1-2) into (1-1) and integrating from $t=0$ to some later time $\tau$,

$\frac{1}{n(\tau)}-\frac{1}{n_{0}}=\frac{\langle\sigma v\rangle}{2} \tau$

Multiplying Equation (1-3) by $n_{0}$ and using the definition of the burn fraction,

$$
\frac{f}{1-f}=\frac{\langle\sigma v\rangle}{2} n_{0} \tau
$$

From Equation 1.4, typically referred to as the Lawson Criterion, we see that the burn fraction increases with the $n_{0} \tau$ product, or confinement. Inertial confinement fusion achieves the $n_{0} \tau$ necessary to consume a significant portion of the fuel through a symmetric compression of the target. The outer shell of the fuel capsule is made of a heavy material, or ablator, that is heated by the energy from the driver. As it expands outward, the fuel is propelled inward, conserving momentum in a rocket-like fashion. Inertia, the tendency of matter to resist changes in its speed or direction, is responsible for two effects. First, the inertia of the fuel during radial compression overcomes the outward pressure due to the rapid increase in density. This compression takes place on a $\sim 10$ ns timescale. Once compressed and heated to a plasma state, the time it takes for the fuel ions to escape is limited by their thermal speed and the radius of the fuel. The second inertial effect, therefore, is that of the fuel mass limiting its own disassembly until 
a significant fraction has undergone the reaction, a process that lasts only tens of picoseconds.

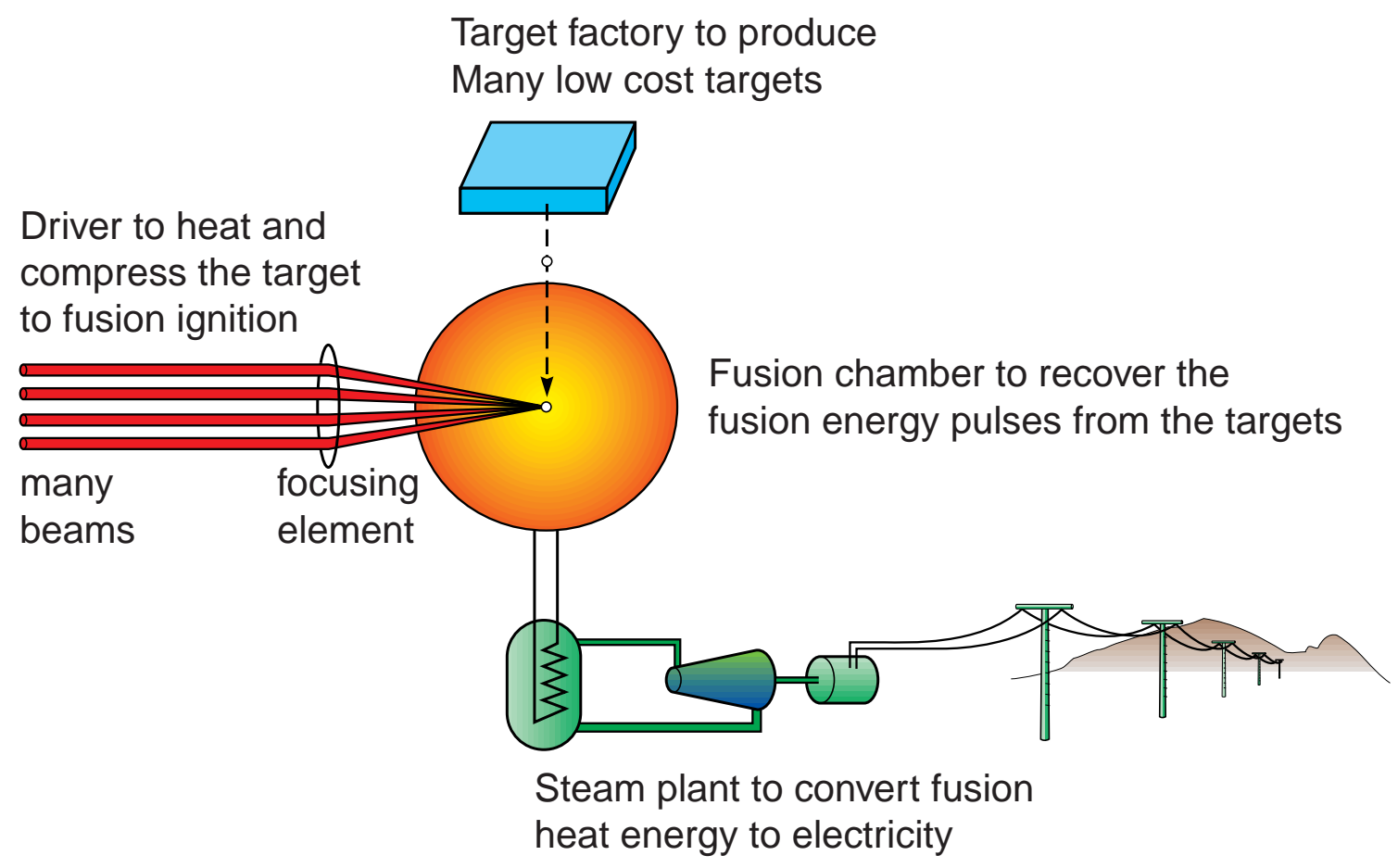

Figure 1.2: Simplified schematic of an IFE power plant

An inertial confinement fusion power plant is shown schematically in Figure 1.2. Components universal to all designs are shown: driver, target factory, reaction chamber, and steam plant. The net electrical power produced $P_{e}$ is related to the gross power $P_{g}$ from fusion by the following equation:

$P_{e}=P_{g}-P_{d}-P_{a}=P_{g}\left(1-f_{p}-f_{a}\right)$

where $P_{a}$ is the power used to run the auxiliary equipment for the steam cycle, and $P_{d}$ is the power required by the driver. $f_{p}$ is the driver's recirculating power fraction, and is related to the driver efficiency $\eta_{d}$, the ratio of fusion yeild to driver energy $G$, the thermal-to-electric conversion efficiency $\eta_{t}$, and the nuclear energy multiplier $M$ by $f_{p}=\frac{1}{\eta_{t} \eta_{d} G M}$ 
The inverse of this quantity, $P_{g} / P_{d}$, is an important metric for the ultimate cost of electricity. Considering the capital cost of a one gigawatt power plant, this ratio needs to be $\geq 5$. (Hogan, 1992). The conversion efficiency for a steam plant is usually $0.35-0.45$, and the nuclear energy multiplier (due to neutron induced nuclear reactions) is typically 1.05-1.15. This means that the $\eta_{d} G$ product, the effectiveness of the driver-target combination, should be at least 10 .

\subsection{Heavy Ion Fusion}

A charged particle beam from an accelerator appears to be a very likely choice for a driver as existing accelerators properly configured appear to be capable of producing the several MJ of energy necessary to achieve the confinement and temperature conditions discussed above. Additionally, machines typically used for high energy physics research have a long life and are reliable over many cycles. Further, the focusing system that delivers the beam energy to the target must be shielded from or impervious to neutrons, x-rays, and target debris. The magnet windings used for focusing of charged particles, as opposed to laser optical elements, can be arranged such that they are shielded from the line of sight of the fusion reaction (Latkowski, 2000).

Symmetry for compression is achieved in one of two ways. In the direct drive scenario, beams enter the chamber through ports that cover enough of the $4 \pi$ solid angle to allow symmetric bombardment of the spherical ablator shell. The other option, indirect drive, involves placing the spherical fuel/ablator pellet inside a casing. This casing, or "hohlraum," converts the energy from the incident ion beams into spherically symmetric x-ray radiation at the fuel capsule.

A schematic illustration of a recent cryogenic HIF target is shown in Figure 1.3. 
This type of target can achieve a gain of 130 from 3.3 MJ of beam energy (CallahanMiller, 2000). At the center of the hohlraum is the fuel pellet, consisting of an outer shell of heavier ablator material, surrounding an inner shell of solid deuterium-tritium fuel. Deuterium-tritium gas fills the remainder of the fuel pellet. The beams strike the ends of the target as depicted by the arrows, depositing its energy in the radiator material, shown in purple. The beam pattern is annular, formed by a ring of $\sim 16$ elliptical beam spots with major and minor radii of $2.78 \mathrm{~mm}$ and $1.0 \mathrm{~mm}$ respectively. The combination of the annular ring of beam illumination and the close proximity of the radiator material allows this target high gain with low incident energy. However, these features are at the expense of a beam spot that must be relatively small by comparison to other target configurations. This is an important trade-off for HIF physics designs.

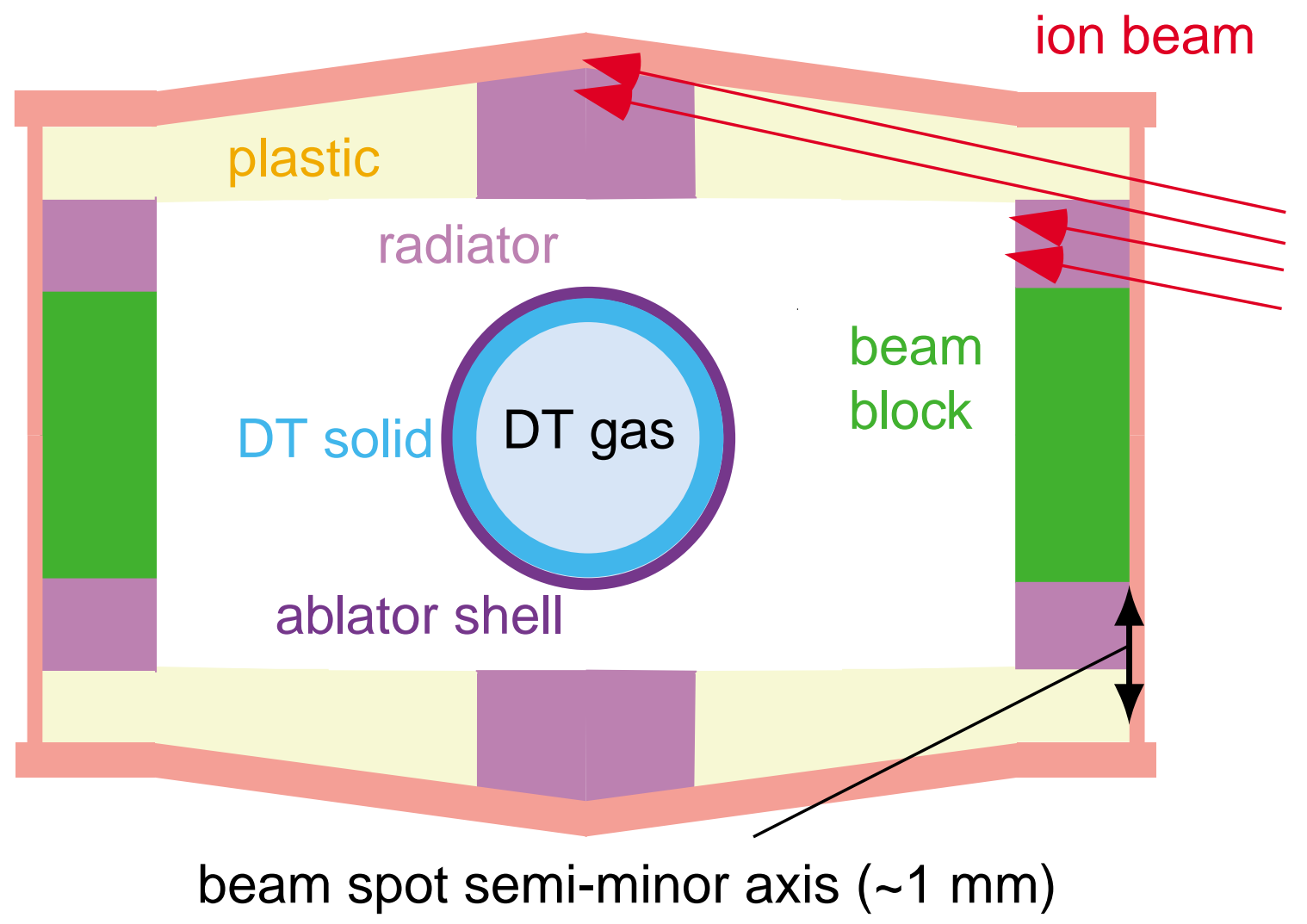

Figure 1.3: Design for a high yield $(G=130)$ indirect drive target 
For efficient conversion to x-rays, it is desirable that the incident ions deposit their energy in a very short distance into the hohlraum converter material. Figure 1.4 is a plot of ion range versus energy for various atomic masses. Typical ICF targets produce highest gains for incident ion ranges between 0.02 and $0.2 \mathrm{~g} / \mathrm{cm}^{2}$ (Bangerter, 1995). If we take the power necessary to drive this type of target to be $4^{*} 10^{14} \mathrm{~W}$ (Hogan, 1995), then heavy ions at $4 \mathrm{GeV}$ require $100 \mathrm{kA}$ total current. By dividing this among 30 beams incident upon the hohlraum one can conceivably transport, and more importantly, focus this current. Because of their longer range, light ions must operate at a lower energy; at $40 \mathrm{MeV}$, they require a total current of $10 \mathrm{MA}$. This is well beyond the limits of conventional accelerator transport unless this current is divided up into hundreds of beams, greatly increasing the complexity of the reactor chamber.

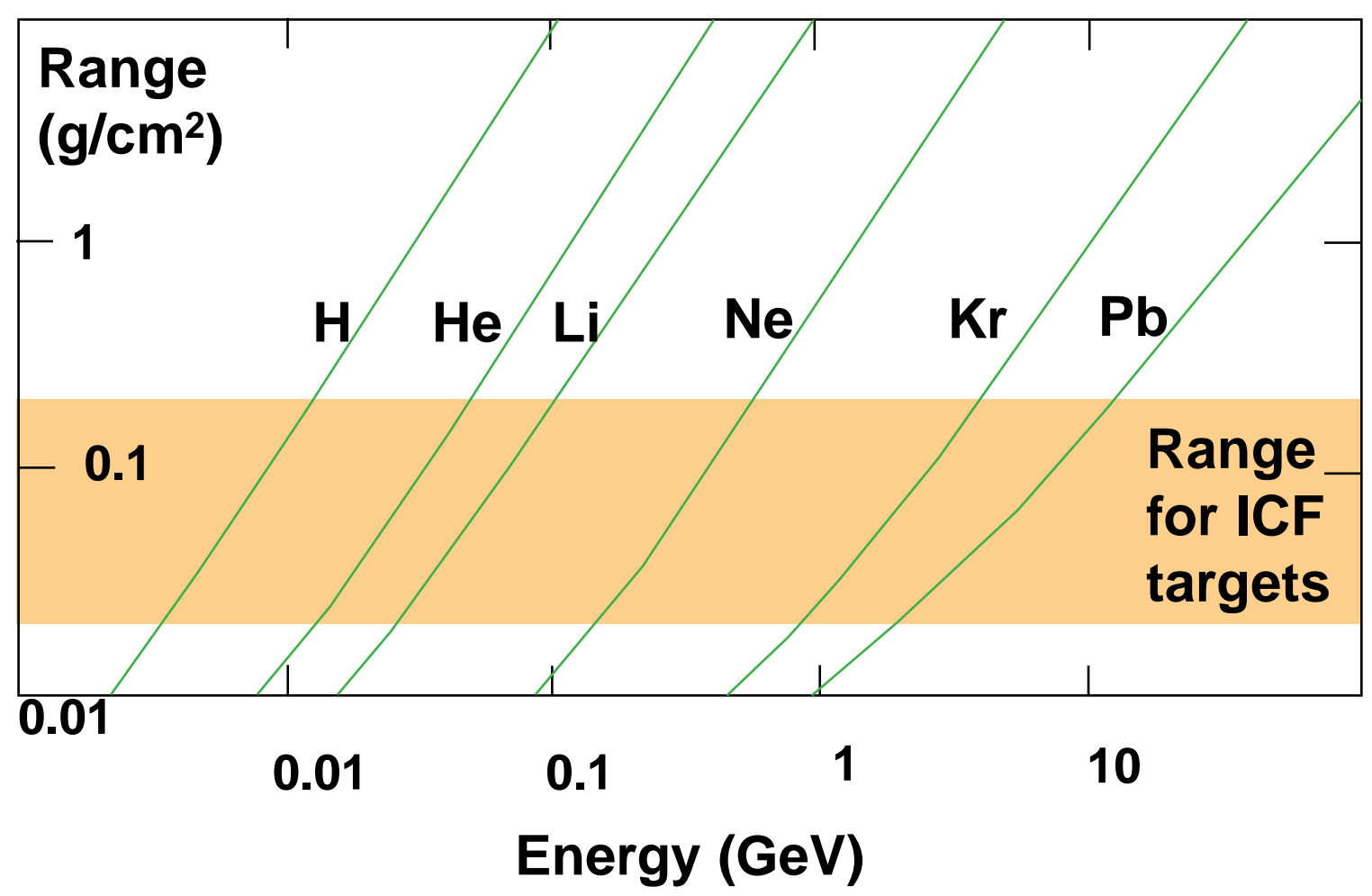

Figure 1.4: Ion range as a function of incident energy for various species 
The target gain $G$ is a function of the target design and the driver energy deposition distribution. On the other hand, the driver efficiency $\eta_{d}$ is purely a function of the accelerator's ability to deliver the drive energy to the target in an economical fashion. While most of the machines for high energy physics research use radio frequency (RF) power to accelerate the particles, it appears that induction acceleration is better suited for HIF purposes for reasons of efficiency. An induction accelerator cell is shown in Figure 1.5. The pulsing circuit is used to induce a time varying magnetic field in the induction core material. Faraday's law then gives the accelerating voltage that appears across the gap due to the $d B / d t$ in the core. In effect, the beam is the secondary of a one-turn transformer.

High current beams can be produced very efficiently in an induction accelerator. The beam current in such an accelerator is a product of the velocity and the line charge density, $\lambda$. Beam injectors operating at $\sim 2 \mathrm{MeV}$ provide the total charge per pulse ultimately needed to drive the target. As the beam velocity increases in the accelerator, the current naturally increases. Because the beam is not highly relativistic, it is quite possible to provide an additional increase in the current by compressing the beam pulse with tailored acceleration waveforms. With the proper longitudinal velocity distribution, the compression can continue to increase the current in a drift section between the end of the accelerator and the target chamber. In this manner, the current necessary for the energy requirements of the target is easily attainable.

In contrast, in an RF accelerator, the current is the product of the charge per RF cycle, or "bucket", and the frequency, both of which are constant. Therefore, the only practical way to increase the current in such an accelerator is through the use of multi- 
turn injection into storage rings, whose bunches are eventually simultaneously delivered to the target. This method adds complexity and therefore cost to the operation of the accelerator. Additionally, the particle "bunch" fills only a fraction of the RF bucket leading maximum efficiencies of $15-20 \%$ for this type of acceleration (Bongardt, 1998). Induction accelerators are therefore the machines of choice for HIF, with configurations able to operate at efficiencies of 25-35\% (Hovingh, 1987). This provides for an $\eta_{d} G$ product well suited to the production of electrical power for gains as low as 50 .

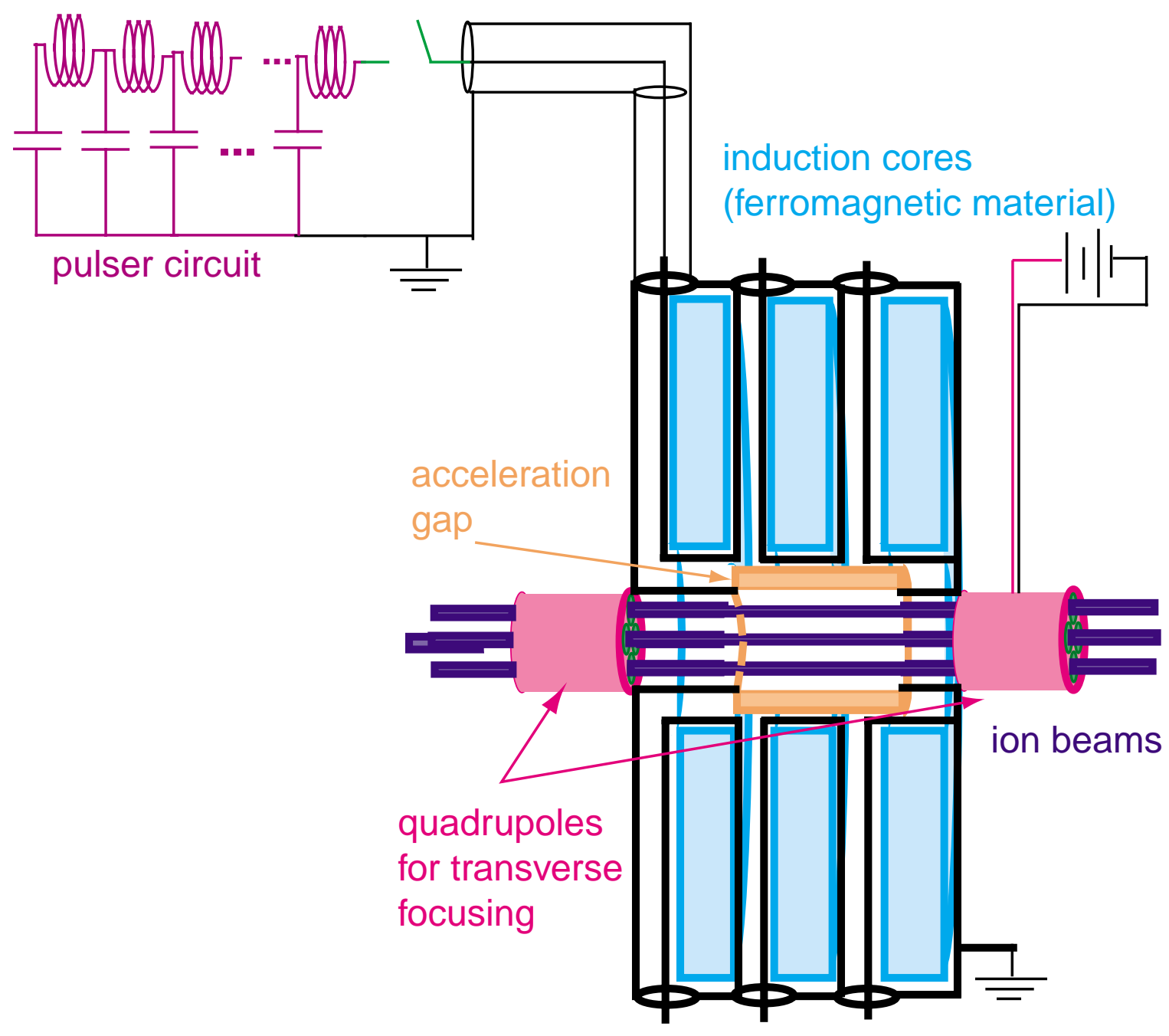

Figure 1.5: Schematic of an accelerating cell in an induction accelerator 


\subsection{The Final Focus Sub-System and the HIBALL-II Design}

By this point in the discussion, the importance of the final focus sub-system is evident. It is the piece of the design that couples the particle accelerator to the fusion fuel. The performance of the final focus must conform to the specifications of both the ion pulse delivered to it and the target it must ignite. Specifically, it must bring the beam to a spot size that meet both the energy and symmetry demands of the target by overcoming the forces within the beam that tend to resist focusing. Because the ions are only marginally relativistic $(\beta=0.2-0.3)$, the magnetic pinch force is weaker than the electric space charge force pushing radially outward on the beam. Additionally, there will be transverse thermal forces, or emittance, that will cause the focal spot to spread.

Since 1976, numerous designs have been proposed for the final focus sub-system. The approaches taken to resolve beam dynamics issues that have arisen serve to put this thesis in context. Therefore, a condensed history of these efforts is described in Appendix A. In general, the concepts listed in the appendix are in the category of ballistic focusing. The focus is accomplished by giving the beam ions entering the target chamber the trajectories they will need to converge to the required spot. This technique assumes there is a good vacuum in the chamber so there is little that would perturb these trajectories or change the charge state of the beam. The driver ion kinetic energy is high enough to significantly weaken the effects of space charge defocusing, causing the focal spot size to be primarily determined by the emittance.

Of concern to the final focus problem is the use of a lower current "prepulse" that is used to pre-heat the radiator material in the hohlraum. This implies that the system of focusing elements must be designed to yield the same focusing results for two separate 
sets of beam parameters. While efforts in this direction are mentioned in Appendix A, an alternate approach is to utilize separate beamlines for the prepulse beams with separate sets of optimized focusing elements.

There are more recent designs for heavy ion driver focusing systems that fall into two other categories. Neutralized, ballistic focusing takes the approach described above, but adds electrons to the converging beam to greatly reduce the space charge and thus the defocusing electric field. The space charge voltage depression of a driver scale ion beam at the final focus can create fields of several $\mathrm{kV} / \mathrm{mm}$ at the vacuum boundary, allowing electrons to be emitted from the grounded beam pipe (Herrmannsfeldt, 2000). Other likely sources of electrons for neutralization are ionization of the background gas by the energetic beam ions and photoionization from the target.

Finally, channel transport is a third category of final focus designs. This encompasses methods that focus in a high background pressure in the chamber using an azimuthal magnetic field. The $B_{\theta}$ could come from an externally triggered discharge ( $\mathrm{Yu}$, 1998), or a "self pinch" in which the beam current is neutralized to a much lesser degree than the space charge (Welch, 1996). The beam converges to a small spot at the edge of the chamber, and the magnetic focusing maintains the small beam radius in the "channel" through the chamber to the target.

The above categories are listed in decreasing order as far as the constraints placed on the ion beam delivered by the accelerator. In particular, the channel transport options significantly reduce the beam port area exposed to radiation from the chamber, easing shielding requirements. However, they are in order of increasing complexity from a technology standpoint, and increasing uncertainty as far as physics issues are concerned. 
Partly for these latter reasons, the Scaled Final Focus experiment is based on the HIBALL-II power plant design, which employs an un-neutralized ballistic focus for a $1.25 \mathrm{kA}$ beam of $10 \mathrm{GeV} \mathrm{Bi}^{+}$ions (Wollnik, 1985). The power plant design is a widely known product of efforts from a consortium of several institutions. The HIBALL-II final focus sub-system, based on a lattice of six magnetic quadrupole elements, was chosen as the final focus model for a power plant costing study performed several years later (Brady, 1987), with the original cost per focusing bemline estimated at \$1.2M in 1984 dollars (Wollnik, 1985, p. 197). Such a system is well suited to an initial experimental verification of the effectiveness of the final focus. It is the first appropriately scaled experimental test of the beam dynamics involved with focusing ions from a heavy ion driver onto the fusion target.

\subsection{Goals For This Work}

Ballistic focusing is still considered to be the "main line" approach to the final focus. Therefore, an effort is needed to test the dynamics of such a design and ensure that an emittance dominated spot can be achieved. By properly scaling the physics parameters that relate particle energy and mass, beam current, beam emittance, and focusing field, the dynamics of a driver scale final focus can be replicated in a small laboratory beam. The scaling calculation is a critical part of the experimental effort, and is described in detail in Chapter 2. The scaled experiment is a true test of the transverse beam physics involved in the focus of a heavy ion beam.

The driver design uses magnets wound from discrete conductors and therefore fields that depart from those of an ideal quadrupole. The identically scaled fields in the experiment reproduce the types of aberrations resulting from these field imperfections. 
Another type of aberration that could significantly affect the focal spot radius is due to deviations from the nominal ion momentum, causing a variation in the focal length produced by the magnet system (Callahan, 1996). These "chromatic" aberrations could result from any residual momentum "tilt" due to compressing the beam for current amplification, or from the momentum spread, or longitudinal emittance, that this compression produces. While the experimental beam is monoenergetic, it is still possible duplicate the effects of the chromatic aberration on the focal spot by considering the beam to represent an off momentum "slice" of the driver beam.

While the previous section explains the choice of modeling an un-neutralized ballistic focus in the scaled experiment, recent consideration of the propagation of the ion beam through the fusion chamber indicates that some degree of neutralization may be unavoidable. The liquid blanket used to capture the heat from the fusion reactions and transfer it to the steam plant will produce vapor as it absorbs the energy of the explosion (Moir, 1991). These residual gasses must be cleared in the period between shots, a time on the order of $100 \mathrm{~ms}$ for the plant to produce power economically (Hovingh, 1987). The gas that is not cleared will most certainly cause ionization both of the gas atoms and beam ions, producing electrons and increasing the space charge defocusing. These issues demand that a neutralized final focus be considered when designing a plant with a competitive cost of electricity.

In fact, some driver and target choices require a high degree $(>95 \%)$ of neutralization of the beam. Reducing the ion energy may not only lower the cost of the accelerator, but it also allows for more flexibility in target design due to the shortening of the ion range. However, these high gain targets typically require smaller spot sizes from 
beams with larger space charge forces. The final goal of this experiment, therefore, is to study the possibility of overcoming the space charge force of a higher current beam with added electrons. At this point it is important to point out that discussions of "neutralization" in reference to the work reported here always imply partial or fractional neutralization of the beam space charge with electrons. $100 \%$ neutralization of the experimental beam is neither expected nor observed. 


\section{Beam Dynamics and Scaling}

\subsection{The K-V Envelope Equation}

As mentioned at the end of Chapter 1, proper scaling of the beam current, external focusing gradients, and emittance with the beam energy and dimensions is critical if we desire to faithfully reproduce the particle trajectories of a full scale driver in a small laboratory experiment. The tool that is used for this scaling is the envelope equation, which gives the trajectory of the edge of the beam as a function of the self and applied fields and the emittance. The equation, along with the distribution function describing a beam that obeys the equation, was first developed by Kapchinskij and Vladimirskij (Kapchinskij, 1959). A derivation is presented here, with the intention of emphasizing the physical basis for each of the terms in the equation. Then, in the next section, these terms are taken individually to extract the experimental parameters from those of the selected driver design.

There are several key assumptions that are incorporated in the envelope equation. The beam charge density is taken to be uniform, filling an elliptical cross section with semi-axes $a$ and $b$. The axial velocity of the particles, $v$, is assumed to be a constant, as is the beam current, $I$. Together they describe a constant line charge density, $\lambda$. Note that these two assumptions lead to linear self fields within the beam. The axial beam dimension is assumed to be much longer that the radii, $l>>a, b$. Finally, the beam is composed entirely of particles with mass $m$ and charge state +1 .

With these assumptions in mind, the equations for the beam's transverse self fields in free space are 


$$
\begin{aligned}
\vec{E} & =\frac{\lambda}{\pi \varepsilon_{0}} \frac{1}{a+b}\left(\frac{x}{a} \hat{x}+\frac{y}{b} \hat{y}\right) \\
\vec{B} & =\frac{\mu_{0} \lambda}{\pi} v \frac{1}{a+b}\left(-\frac{y}{b} \hat{x}+\frac{x}{a} \hat{y}\right)
\end{aligned}
$$

and the equation of motion in the $\mathrm{x}$ direction for a single particle is

$\gamma m \ddot{x}=e\left(E_{x}-v B_{y}\right)$

so that

$$
\ddot{x}=\frac{e}{\gamma m}\left[E_{x}(z)-v B_{y}(z)\right]_{e x t}+\frac{e}{\gamma m} \frac{\lambda}{\pi \varepsilon_{0}} \frac{1}{a+b}\left(1-\frac{v^{2}}{c^{2}}\right) \frac{x}{a}
$$

where the first term denotes the external focusing force assumed to be from a quadrupole field. For an assumed constant axial velocity, the time derivative is converted to a derivative with respect to axial position $(\mathrm{z})$

$$
x^{\prime \prime}=k_{x}(z) x+\frac{e I}{\pi \varepsilon_{0} m(\beta \gamma c)^{3}} \frac{x}{a(a+b))}
$$

and the external fields are represented by the function $k_{x}$. The external term is written proportional to $\mathrm{x}$ as quadrupole focusing is assumed, and $k_{x}=\frac{q E^{\prime}}{\gamma m v^{2}}, \frac{q B^{\prime}}{\gamma m v}$ respectively, for electrostatic and magnetic focusing gradients.

Equation 2-5 can be written in the form of Hill's equation, $x^{\prime \prime}+\bar{K} x=0$, having the solution

$$
x=A_{x} \cdot w(z) \cos \Psi(z), \Psi(z)=\psi(z)+\phi, \text { and } \psi^{\prime}(z)=1 / w^{2}(z)
$$

with $A_{x}$ and $\phi$ constants. Substituting 2-6 back into Hill's equation, we get

$$
w^{\prime \prime}+\bar{K} w-1 / w^{3}=0
$$

From 2-6, we also see that $x_{\max }=a=A_{x} w$, and $x_{\max }^{\prime}=a^{\prime}=A_{x} w^{\prime}$. If we write the identity $\sin ^{2} \Psi+\cos ^{2} \Psi=1$, we find the relation 
$\frac{x^{2}}{w^{2}}+\left(w x^{\prime}-x w^{\prime}\right)^{2}=A_{x}^{2}$

This is the equation of an ellipse in X-X' space with area $A_{x}{ }^{2}$. We can consider the area of the ellipse that is formed by the outermost particle orbits and define it as $\varepsilon_{x}=A_{\max }^{2}$, where $\varepsilon_{x}$ is the emittance of the beam in the $\mathrm{x}-\mathrm{X}^{\prime}$ plane.

At this point the $\mathrm{K}-\mathrm{V}$ distribution function mentioned above can be introduced:

$f_{K V}=f_{0} \delta\left(\frac{A_{x}^{2}}{\varepsilon_{x}}+\frac{A_{y}^{2}}{\varepsilon_{y}}-1\right)$

This is a distribution in four dimensional phase space in which the self fields are linear.

We can see this by inserting Equation 2-8 and the corresponding relation for the $y-y^{\prime}$

plane, and integrating the distribution function over the transverse momentum to find the density:

$\rho=q \iint f_{K V} d x^{\prime} d y^{\prime}=q \cdot f_{0} \pi \frac{\varepsilon_{x} \varepsilon_{y}}{a b}$

where the relations $a=\sqrt{\varepsilon_{x}} w$ and $b=\sqrt{\varepsilon_{y}} w$ have been used in the second equality. Note that the charge density of the distribution is independent of $\mathrm{x}$ or $\mathrm{y}$. Another feature of the $\mathrm{K}-\mathrm{V}$ distribution is that it is a solution of the time independent Vlasov equation, and is therefore a stationary distribution when the external focusing forces are linear (Reiser, $\mathrm{p}$. 341-7).

Returning to Equation 2-7, we can now write this expression in terms of the beam radius $a$ $a^{\prime \prime}+\bar{K} a-\frac{\varepsilon_{x}^{2}}{a^{3}}=0$

If we now consider the self field term in Equation 2-5, we can define the dimensionless perveance as 
$Q=\frac{e I}{2 \pi \varepsilon_{0} m(\beta \gamma c)^{3}}$

Then, using Equation 2-12 and the expression for $\bar{K}$ from Equation 2-5 in Equation 2-11, we obtain

$a^{\prime \prime}=k_{x}(z) a+\frac{2 Q}{a+b}+\frac{\varepsilon_{x}^{2}}{a^{3}}$

Equation 2-13 is the beam envelope equation in its most useful form. It may now be used to develop the relations that scale the parameters of the driver design to the values used in the experiment. It will also be used to calculate an expected focal spot size from the beam parameters measured at the end of the focusing system. The relative strengths of the various terms as the beam traverses the focusing system are discussed in detail in conjunction with the measurements in Chapter 6.

It is helpful to define an rms expression for $\varepsilon_{x}$ that can be used to obtain a value for the emittance from an experimental measurement of the phase space. This expression can be derived by first defining the rms envelope radius $a^{2}=4\left\langle x^{2}\right\rangle$ and then inserting into the second derivative the equation of motion for a particle at the outer edge of the beam. The average over the total number of particles $\mathrm{N}$ is defined by

$\langle X\rangle=\frac{1}{N} \sum_{i=1}^{N} X_{i}$

By taking derivatives of the rms envelope, we can write

$\left(a^{\prime}\right)^{2}+a a^{\prime \prime}=4\left\langle\left(x^{\prime}\right)^{2}\right\rangle+4\left\langle x x^{\prime \prime}\right\rangle$

solving for $a^{\prime \prime}$ and inserting $a^{\prime}$ in terms of $a$,

$a^{\prime \prime}=\frac{4}{a}\left[\left\langle\left(x^{\prime}\right)^{2}\right\rangle+\left\langle x x^{\prime \prime}\right\rangle\right]-\frac{16\left\langle x x^{\prime}\right\rangle^{2}}{a^{3}}$

Inserting Equations 2-5 and 2-12, 
$a^{\prime \prime}=\frac{4}{a}\left[\left\langle\left(x^{\prime}\right)^{2}\right\rangle+k_{x}\left\langle x^{2}\right\rangle+\frac{2 Q}{a(a+b)}\left\langle x^{2}\right\rangle\right]-\frac{16\left\langle x x^{\prime}\right\rangle^{2}}{a^{3}}$

and using the relation $a^{2}=4\left\langle x^{2}\right\rangle$, we get

$a^{\prime \prime}=\frac{16\left\langle\left(x^{\prime}\right)^{2}\right\rangle\left\langle x^{2}\right\rangle}{a^{3}}+k_{x} a+\frac{2 Q}{(a+b)}-\frac{16\left\langle x x^{\prime}\right\rangle^{2}}{a^{3}}$

Finally, comparing Equation 2-18 to Equation 2-13, we can define an "edge" emittance that is equivalent to the transverse phase space area of the beam in terms of rms quantities:

$\varepsilon_{\text {xedge }}=4 \varepsilon_{\text {xrms }}=4 \sqrt{\left\langle\left(x^{\prime}\right)^{2}\right\rangle\left\langle x^{2}\right\rangle-\left\langle x x^{\prime}\right\rangle^{2}}$

Because the second moments and cross moment are easily obtained from the

experimental measurement, all emittance results quoted in this work are calculated using this expression.

To bring physical meaning to this quantity that falls out of the solution to Equation 2-5, we can relate the emittance to the transverse temperature of the beam ions. Re-writing the expression for the rms emittance in eqation 2-19 for a non-relativistic beam,

$\varepsilon_{x r m s}=\sqrt{\left\langle\left(x^{\prime}\right)^{2}\right\rangle\left\langle x^{2}\right\rangle-\left\langle x x^{\prime}\right\rangle^{2}}=x_{r m s} x_{r m s, t h}^{\prime}=x_{r m s} \frac{v_{r m s, t h}}{\beta c}$

The term involving $x x^{\prime}$ in the first equality is a correlation between the phase space coordinates that results from a beam that is either converging or diverging in the $\mathrm{x}$ direction after having passed through a lens, for example. What remains after this quantity is subtracted represents the random, thermal component of the transverse rms velocity distribution. Assuming a Maxwellian intrinsic velocity distribution for a round 
beam of radius $a$ and transverse temperature $T_{\perp}$, Equations (2-19) and (2-20) can be combined to write the edge emittance as

$$
\varepsilon_{x}=\frac{2 a}{\beta} \sqrt{\frac{k_{B} T_{\perp}}{m c^{2}}}
$$

\subsection{Scaling Relations}

The relations that are used to select the appropriate values for the experimental parameters are arrived at by identifying the parameters that are limited by the available experimental facilities, and adjusting the remaining quantities to preserve the physics of interest as contained in Equation 2-13. The hardware constraints are the physical dimensions of the experiment, for which we have chosen a one-tenth scale, the kinetic energy of the ions, which is $160 \mathrm{keV}$ as compared to $10 \mathrm{GeV}$ in the design, and the emittance, which is primarily determined by the ion source. These three are not entirely independent; the emittance is affected by the dimensional scaling, and it was therefore important to select a source design that produced a very low emittance for use in this much smaller scale experiment. Then, the beam current and the strengths of the focusing fields are adjusted to reproduce the particle trajectories.

To use the envelope equation as a guide for proper scaling, we first note that the equation has dimensions of inverse length. Therefore, each of the terms in the equation need to be adjusted by the inverse of the dimensional scale factor:

$\frac{\text { term }_{e}}{\text { term }_{d}}=\frac{\text { length }_{d}}{\text { length }_{e}}=D=10$

where the subscript $e$ refers to the experiment and $d$ to the design. First we scale gradient term using the expression for $k_{x}$ associated with magnetic focusing from the previous section. Inserting this into Equation 2-22 we derive the relation for the magnetic field 
$\frac{k_{e} a_{e}}{k_{d} a_{d}}=\frac{\left(B_{e} / a_{e}\right)(m \beta \gamma)_{d}}{\left(B_{d} / a_{d}\right)(m \beta \gamma)_{e}} \frac{a_{e}}{a_{d}}=D$

Repeating the process for the other terms,

$\frac{Q_{e} / a_{e}}{Q_{d} / a_{d}}=\frac{I_{e}\left(m \beta^{3} \gamma^{3}\right)_{d}}{I_{d}\left(m \beta^{3} \gamma^{3}\right)_{e}} \frac{a_{d}}{a_{e}}=D$

$\frac{\varepsilon_{e}^{2} / a_{e}^{3}}{\varepsilon_{d}^{2} / a_{d}^{3}}=D$

yields scaling relations for the beam current and emittance. Keeping in mind that the ratio of the design radius to the experimental radius is just $\mathrm{D}$, the experiment-to-design ratios for these parameters can then be solved for explicitly:

$$
\begin{aligned}
& B_{e}=B_{d} \frac{(m \beta \gamma)_{e}}{(m \beta \gamma)_{d}} D \\
& I_{e}=I_{d} \frac{\left(m \beta^{3} \gamma^{3}\right)_{e}}{\left(m \beta^{3} \gamma^{3}\right)_{d}} \\
& \varepsilon_{e}=\varepsilon_{d} \frac{1}{D}
\end{aligned}
$$

\subsection{Summary of Scaled Parameters}

The effect of the scaling is to produce a set of manageable parameters for a small laboratory experiment. The HIBALL-II design used $\mathrm{Bi}^{+}$ions (mass 209) at $10 \mathrm{GeV}$, while the experiment uses $\mathrm{Cs}^{+}$(mass 133) at $160 \mathrm{keV}$. This results in a ratio of $\beta_{\text {driver }} / \beta_{\text {ex }}=200$. To maintain the dimensionless perveance at $1.1 \times 10^{-5}$, the value for the design at the final focus energy and current, the current is scaled from $1.25 \mathrm{kA}$ to $95 \mu \mathrm{A}$; therefore the magnet fields need only be a few hundred gauss. Any further reduction in the experimental ion kinetic energy, however, would have resulted in a value for the scaled experimental current that would have posed a difficulty for the beam diagnostics. The emittance must be reduced proportional to the dimensional scaling, from $30 \pi$ to $3 \pi$ 
$\mathrm{mm}$ mrad. Because the beam trajectories are identical, $9 \mathrm{mr}$ is the final convergence angle for both cases.

These results are summarized in Table 2-1. The formulae in the table separate Equations 2-25, 2-26, and 2-27 into their explicit dependencies on the energy and dimensional scaling.

\begin{tabular}{|l|l|l|l|}
\hline term & field & emittance & current \\
\hline $\begin{array}{l}\text { energy } \\
\text { scaling }\end{array}$ & $B_{e}=\frac{\beta_{e} \gamma_{e} m_{e}}{\beta_{d} \gamma_{d} m_{d}} B_{d}$ & $\varepsilon_{e x}=\varepsilon_{\text {driver }}$ & $I_{e}=\frac{\beta_{e}^{3} \gamma_{e}^{3} m_{e}}{\beta_{d}^{3} \gamma_{d}^{3} m_{d}} I_{d}$ \\
\hline $\begin{array}{l}\text { dimensional } \\
\text { scaling }\end{array}$ & $B_{e} \propto \boldsymbol{D}^{1}$ & $\varepsilon_{e} \propto D^{-1}$ & $I_{e} \propto D^{0}$ \\
\hline HIBALL II & 0.65 to 1.8 Tesla & $\begin{array}{l}30 \pi \mathrm{mm} \\
\text { mrad }\end{array}$ & $1.25 \mathrm{kA}$ \\
\hline $\begin{array}{l}\text { Scaled } \\
\text { Experiment }\end{array}$ & $\begin{array}{l}200 \text { to } 600 \\
\text { Gauss }\end{array}$ & $3 \pi \mathrm{mm} \mathrm{mrad}$ & $95 \mu \mathrm{A}$ \\
\hline
\end{tabular}

Table 2-1 Parameters of the design and experiment as scaled by the envelope equation 


\section{Description of the Experiment}

The main components of the Scaled Final Focus Experiment include an ion source, an electrostatic section for the selection of the scaled current and preparation of the beam, and a magnetic section that replicates the full scale design. These components are enclosed by a vacuum system accommodating numerous feedthroughs for electrical connections and beam diagnostics. A high voltage pulser used to generate the beam sits adjacent to the source enclosure. The hardware used to produce, transport, and focus the beam is described in Sections 3.1-3.3, and the types and locations of the various diagnostics are explained in Section 3.4.

\subsection{Vacuum System and Beam Pulse Generator}

The components of the final focus system are contained in a vacuum enclosure consisting of several aluminum tanks, pumping devices, a connecting beam pipe, and an isolation gate valve. A photograph of the enclosure is shown in Figure 3.1, and a to-scale schematic is shown in Figure 3.2. Each of the large aluminum vacuum tanks had previously been used in an experiment studying electrostatic transport of a heavy ion beam (Tiefenback, 1985). The numerous ports allow for flexibility in the diagnostic probe locations and ease of viewing through transparent lexan covers. The locations of the beam diagnostics are shown along with their station names, D1 through D5. The large cross section of the tanks was originally designed to provide for the possible inclusion of cryopumping panels (Tiefenback, 1985), and allows easy access to both electrostatic and magnetic focusing elements for installation and alignment. A pressure of $2-4 \times 10^{-7}$ torr was typically achieved with a combination of five turbo pumps, three 
cryo pumps, and two liquid nitrogen cold traps, as indicated in the figure. The effect of the background gas density on the beam is discussed in Chapter 7.

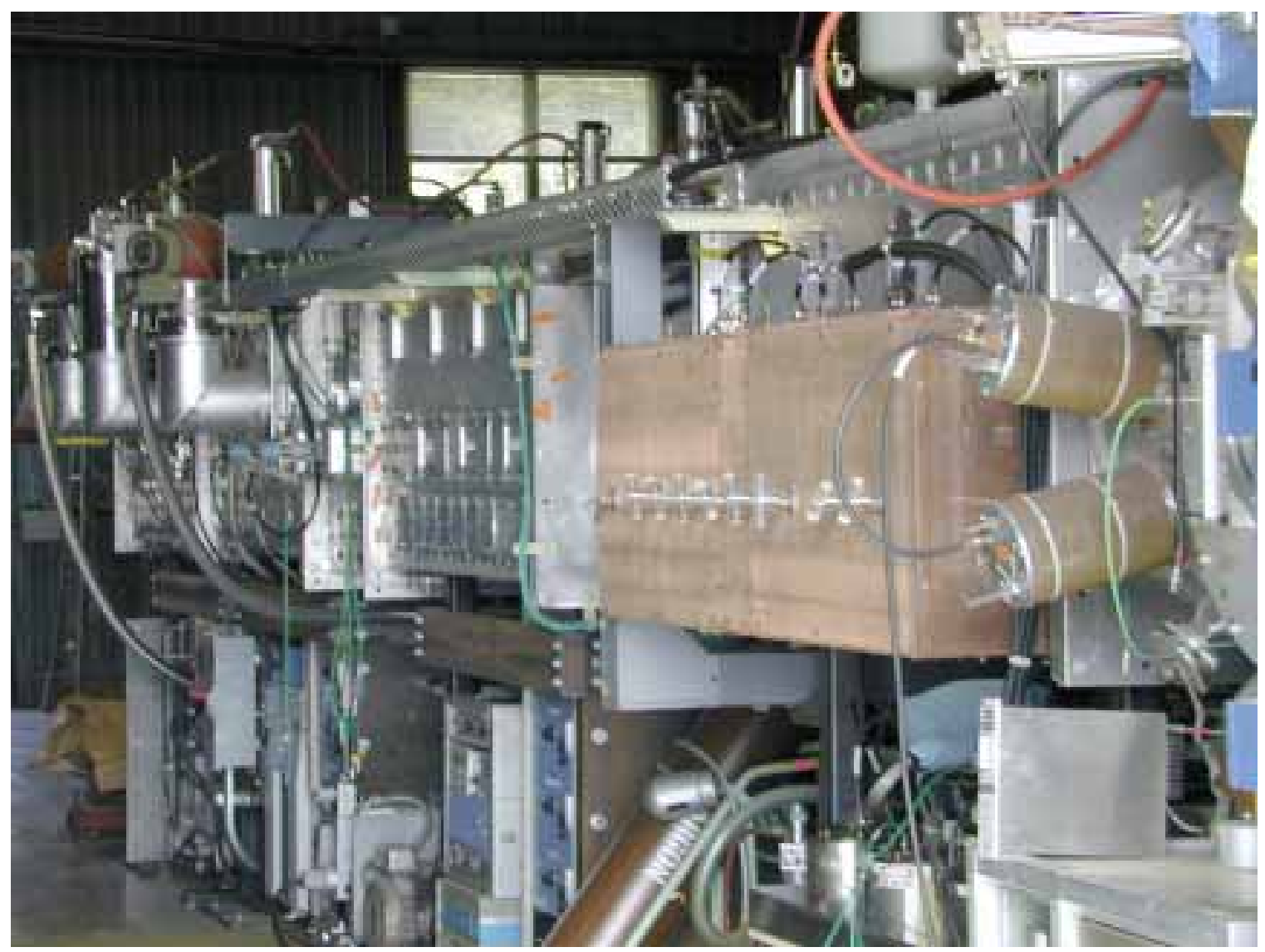

Figure 3.1: Vacuum tanks housing the experiment. The electrostatic section is in the foreground, with the magnetic section in the background to the left.

The pulsed high voltage that accelerates the ions from the source to form a beam is delivered by a Marx generator. This type of pulse generator uses a series of stages, with each stage a series combination of a capacitor and a spark gap with a resistor in parallel. Prior to the pulse, the capacitors are each charged by a common power supply through the resistor network. The spark gaps reside in a single pressurized column enabling them to be triggered simultaneously. With the trigger, the spark gaps close and the voltage on each stage is added. The sum is applied to the ion source cathode with the anode held at ground to extract the beam until an auxiliary spark gap is fired to short the 
high voltage to ground and terminate the beam pulse. Also present in the Marx generator enclosure is a capacitive divider that is used to monitor the voltage pulse. The enclosure is filled with transformer oil to improve the voltage holding capabilities of the components.
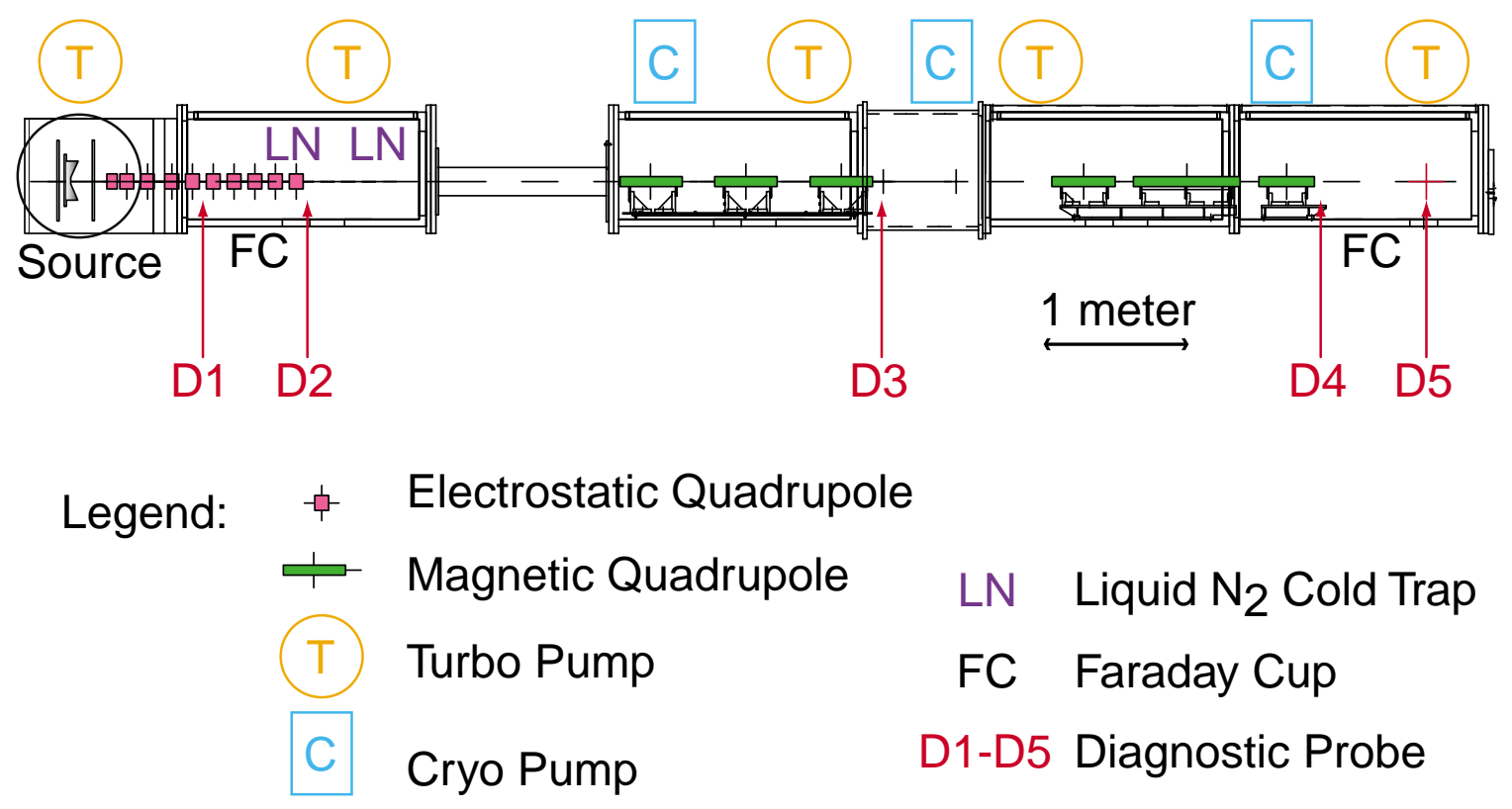

\section{Figure 3.2: Layout of the Scaled Final Focus Experiment}

The voltage as a function of time at the beginning of the Marx pulse is a direct result of the effective electrical circuit seen by the end of the Marx column. The shape of this pulse can lead to a current fluctuation at the head of the resulting ion beam. This distortion of the beam current is produced by space charge transients as the head of the pulse traverses the diode. A functional form for the voltage rise that eliminates the oscillations can be arrived at by paying close attention to the potential at the charge boundary that is the head of the beam (Lampel, 1983). This 1D approximation matches the rise time of the voltage pulse to the transit time of an ion across the diode.

This is not the case for the pulse generated for the Final Focus experiment, as the rise time is considerably slower than the transit time. The resulting current spike 
appearing at the head of the beam pulse does not adversely affect the measurements, however. In fact, the extra resistance and capacitance that cause the slow rise time serve to dampen oscillations in the voltage pulse that are amplified in the beam current pulse. This provides a longer fraction of the full pulse width during which the beam current and voltage are steady state. The total length of the pulse $(10 \mu \mathrm{s})$ provides a 5-6 $\mu$ s period of constant current during which the beam data can be evaluated. The maximum pulse duration is ultimately limited to $\sim 15 \mu$ s by the voltage holding capability of the insulators that support the diode.

\subsection{Electrostatic Focusing Section}

The electrostatic focusing section pictured in Figure 3.3 receives the beam from the ion source, described in the next chapter, and delivers it to the magnetic focusing section with the appropriate beam envelope parameters. This section is 1.6 meters long and consists of a lattice of fifteen electrostatic quadrupoles (ten are used, the last five are at ground) located in the source tank and the first large beam line tank. Positive potential is applied to the either a vertical or horizontal element pair while the opposing pair is tied to ground. The change in voltage from pole to pole provides the quadrupole symmetry and achieves the desired focusing gradient $k$, e.g. $\frac{\partial E_{x}}{\partial x}=k x$, and $\frac{\partial E_{y}}{\partial y}=-k y$. These quadrupoles were also used in a previous ion beam experiment designed to transport $\sim 20$ $\mathrm{mA}, 200 \mathrm{keV} \mathrm{Cs}+$ ions. Here it was desired to keep the lattice period short so as to send the beam through as many periods as possible. The elements are 4 inches long with a 2 inch gap between elements and a 1 inch bore radius, resulting in a ratio of 1:2 for the aperture versus the length. The pole tips were designed to produce a gradient that is 


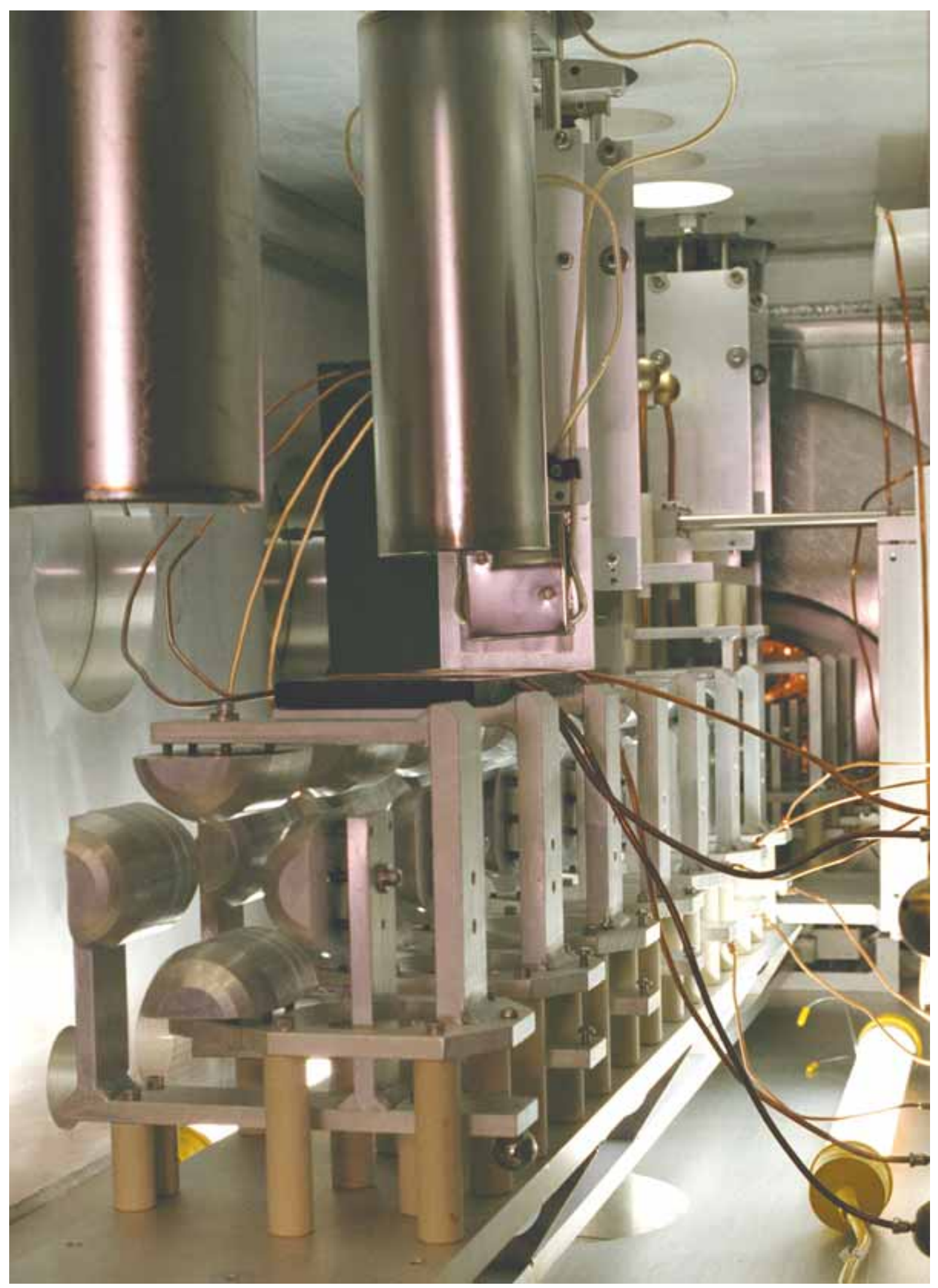

Figure 3.3: Electrostatic transport section. The beam direction is from the background at left to the foreground at right. D1 and D2 diagnostics are visible at the top of the figure. 
sinusoidal in $z$ to preserve the linearity of the focusing field in spite of the large aspect ratio (Tiefenback, 1986). They are presently used to prepare the beam for the magnetic final focus system and provide satisfactory matching of the beam parameters in the short distance from the source to the beginning of the first long drift section.

Because it is desired to apply independent focusing voltages to each of the quadrupoles, the horizontal ports in the first large tank are fully occupied by high voltage feedthroughs. Diagnostics mounted in the vertical ports measure beam parameters along the vertical axis. With the exception of the second quadrupole, the field gradients in the quadrupoles are created with one pair of elements at positive high voltage, and the other pair at ground. The feedthroughs are configured in such a way that the polarity of each quadrupole can be easily switched with the system under vacuum. By switching the polarities of the entire lattice and measuring again the vertical phase space of the beam, a measurement of the effects of the focusing solution on the beam in both transverse directions can be made with only the vertically driven diagnostic paddles. This method is not sensitive to any initial asymmetries in the beam distribution in the horizontal plane. For an axisymmetric beam delivered from the source, however, the method provides a satisfactory means for determining the beam envelope parameters and transverse emittances delivered by the electrostatic focusing solution.

In order to select the desired beam current for the magnetic final focus section, it is necessary to aperture the beam to a small fraction of its radius. Immediately after the diode, an aperture wheel contains graphite apertures as well as an empty slot that allows the full beam current $(5.8 \mathrm{~mA})$ from the source to pass. $1.0,1.5$, and $2.0 \mathrm{~mm}$ diameter openings are used for $\sim 95, \sim 225$, and $\sim 400 \mu \mathrm{A}$ beam currents, respectively. The wheel is 
electrically isolated and biased, which together with the choice of graphite for the aperture material helps suppress secondary electrons.

\subsection{Magnetic Focusing Section}

The magnetic focusing section is 8.2 meters long, including the initial drift from the last electrostatic element and the final drift to the focal spot diagnostic. The middle drift between the third and fourth magnets has been shortened with respect to the HIBALL-II design as the bending magnets that allow for the neutron dump are not included. The six magnets that compose the final focus system are shown in place in the vacuum tank in Figure 3.4.

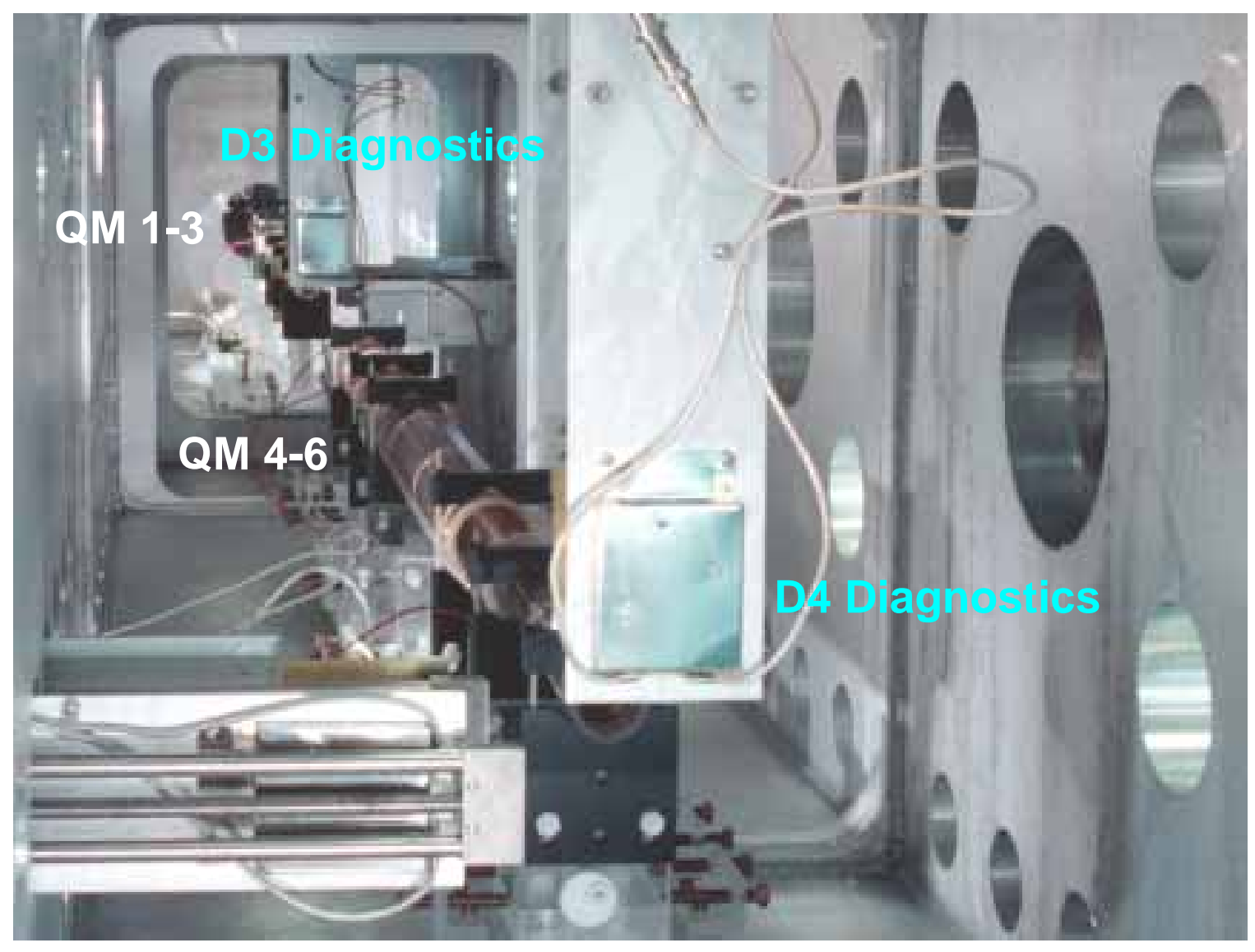

\section{Figure 3.4: Magnetic Focusing Section Hardware}

The magnets are iron-free, pulsed quadrupoles, and consist of two layers of 
copper windings in an azimuthal distribution that best approximates a $\cos (2 \theta)$ surface current. The windings were epoxied into groves cut into cylindrical Lexan shells. Their dimensions are scaled exactly from the HIBALL-II report (Wollnik, 1985). Figure 3.5 shows two of the magnets after fabrication as well as a cross section with the details of the conductor placement. Each of the magnet inner diameters is lined with a grounded metal foil to simulate the beam pipe in the report's focusing system.
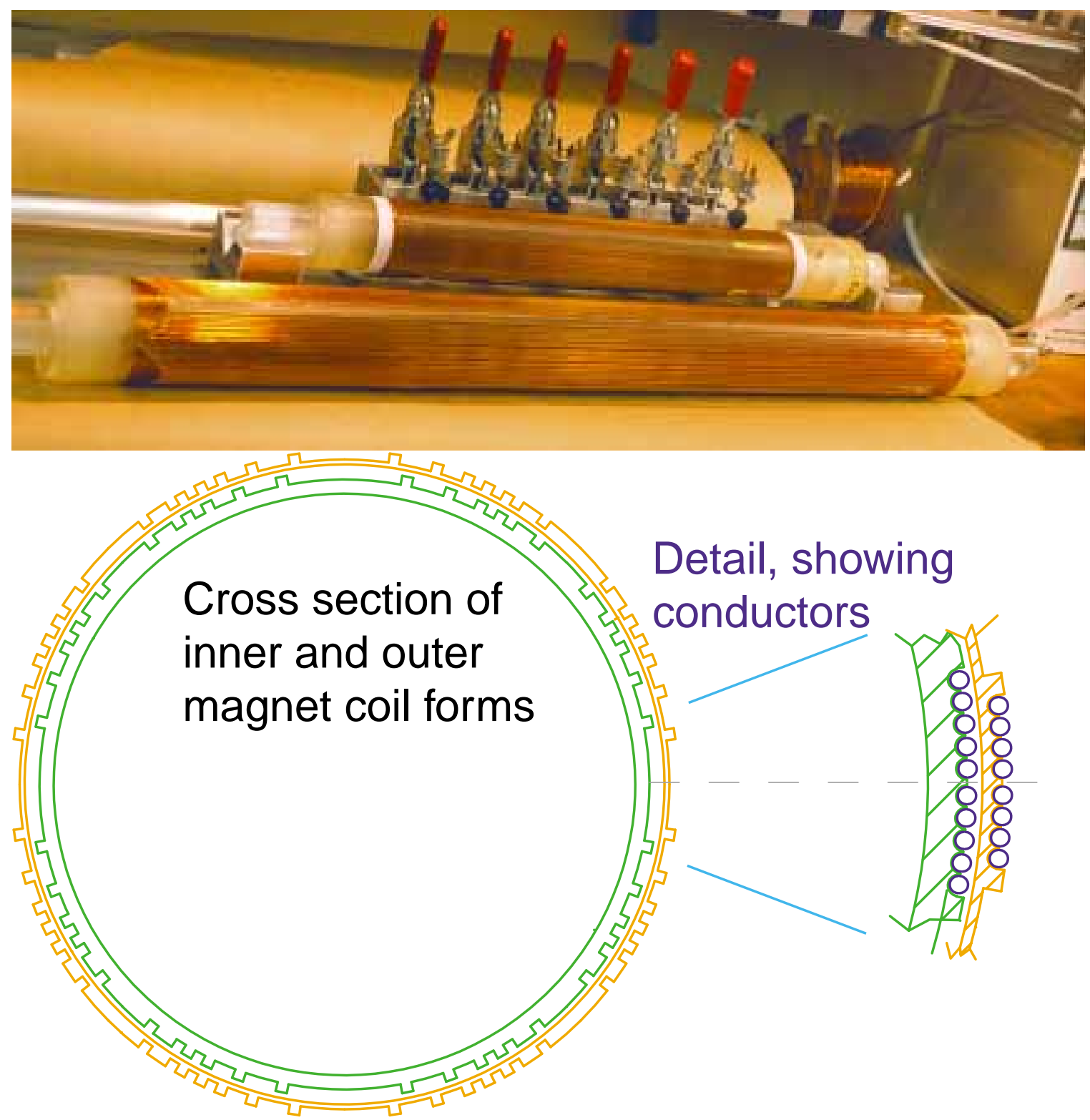

Figure 3.5: The final focus magnetic quadrupoles 
The magnets are powered by pulsers that deliver a sinusoidal pulse approximately $1 \mathrm{~ms}$ in length, with a 50-60 $\mu$ s region at the peak of the pulse during which the current is changing by less than $1 \%$. The total length of time the beam takes to traverse all six magnets is $20 \mu \mathrm{s}$; the magnetic field is essentially constant in time in the presence of the beam. A schematic of the pulser circuit is shown in Figure 3.6. As each magnet has a rise time characteristic of its inductance, the pulsers must be triggered separately to synchronize the focusing fields with the beam. The data acquisition system triggers a set of delay generators in order to properly time the pulses of the individual magnets. The longest delay is then used to trigger the beam pulse when the magnet currents have reached their peaks.

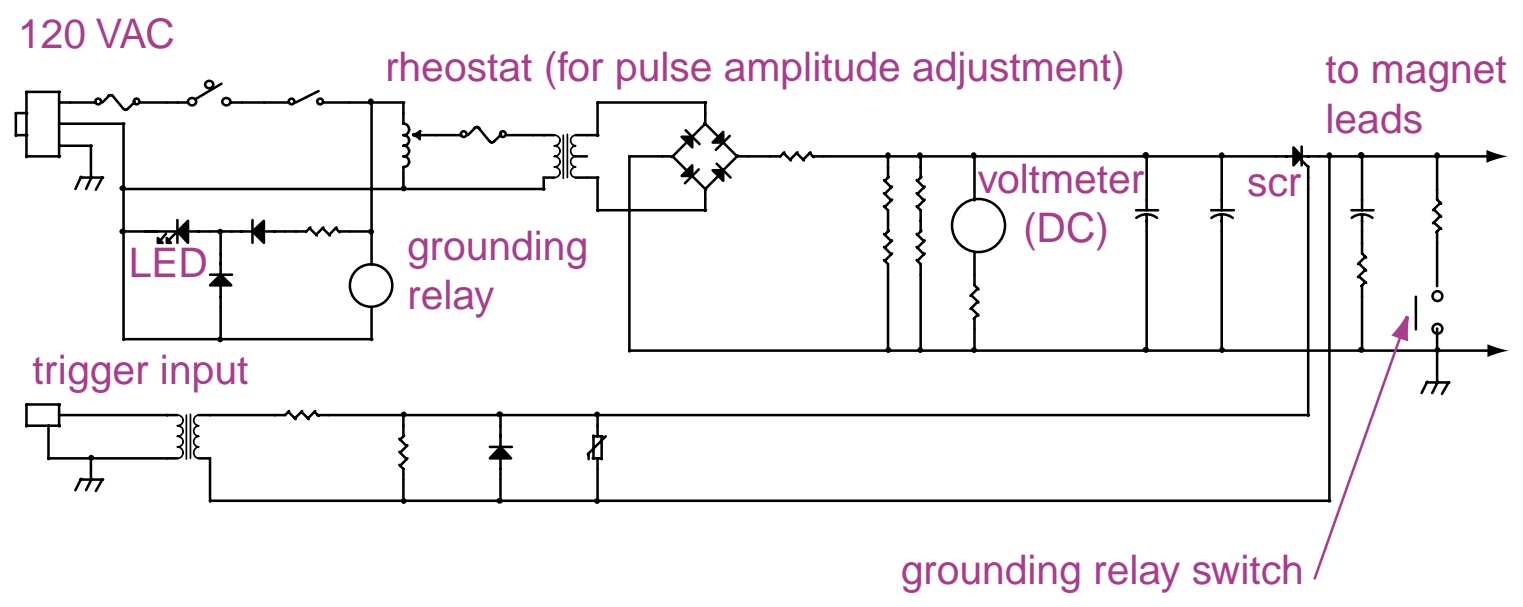

Figure 3.6: Magnet Pulser Schematic

Figure 3.2 shows the D4 diagnostic station, located after the last magnetic quadrupole. Co-located with the horizontal diagnostic probe is a narrow loop of $75 \mu \mathrm{m}$ tungsten wire designed to be inserted into the beam path. This loop is heated with current from a positive DC power supply to approximately $2500 \mathrm{~K}$ to provide a source of cold $(\sim 0.2 \mathrm{eV})$ electrons for neutralization of the beam. The hot loop is shown in Figure 3.7. A potential of approximately 10 volts develops across the ends of the filament due to its 


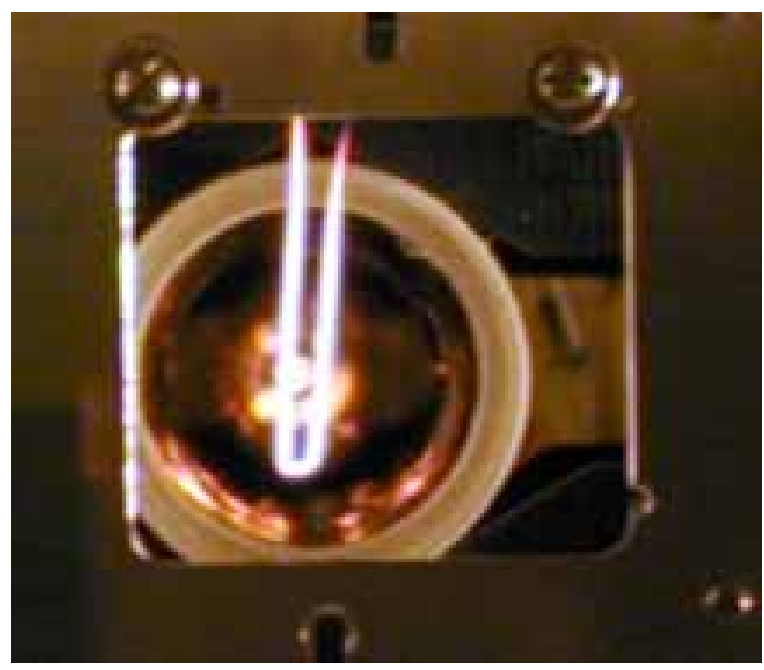

Figure 3.7: Hot filament viewed from downstream. Shown is the bore of the last magnetic quadrupole, and the ion source in the background. finite resistance. To prevent this potential from trapping the electrons at the filament, The DC power supply is switched off with a solid state relay just prior to the arrival of the beam. The filament remains hot enough to emit electrons, but there is no filament potential to keep the electrons from being attracted by the space charge potential of the beam. The switching signal for the relay is simultaneous with

the trigger of the first magnet pulser, $450 \mu \mathrm{sec}$ before the Marx pulse is triggered. The voltage at the relay was measured to go to zero in approximately $100 \mu \mathrm{sec}$, ensuring that the electrons would be free to neutralize the beam.

\subsection{Beam Diagnostics}

Several sets of time-resolved beam diagnostics are mounted to the vacuum system to monitor the transported current and evolution of the phase space. Their locations are indicated in Figure 3.2. Two factors influence the choice of locations of these devices. First, it is desired to have the ability to examine the beam at an interval fine enough to readily determine the source of any undesired effects. Additionally, the shorter sections of the experiment between sets of diagnostic probes can be easily modeled with a beam envelope code. The results from the code are used to make corrections to the strengths of the included focusing elements, and these corrections bring the downstream beam 
parameters closer to what is desired at the beginning of the next section. This process is described in detail in Chapter 5.

There are two Faraday cups for measurement of the total beam current. These cups are designed with a ratio of depth to aperture radius $\geq 2$. This allows complete suppression of secondary electrons from the collector with a $\sim 400 \mathrm{~V}$ bias, and are typically referred to as "deep" Faraday cups. One can be inserted in place of the $7^{\text {th }}$ and $8^{\text {th }}$ electrostatic quadrupoles and the other into the drift after the last final focus magnet. Comparison of the two measurements yields the transmission through the final focus lattice. By adjusting the focusing strengths of the first five electrostatic quadrupoles, the full unapertured beam current may be measured in the first deep Faraday cup. This is the primary method of ensuring that the ion source and diode are delivering a repeatable ion beam profile with a broad, constant current region with amplitude variations of $<0.5 \%$ from pulse to pulse.

The majority of the diagnostic probes mounted to the system consist of pairs of slit paddles. The downstream paddle in each pair has a shallow $(\sim 1 \mathrm{~cm}$ deep) biased collector cup mounted to the back of the slit. An isolated mesh is located between the slit and the collector, as shown in Figure 3.8. Typically, the grid is biased positive, and the collector negative, to amplify the incident ion signal with current from the secondary electrons. The resulting signal is sampled through a coupling capacitor and a low pass filter and amplified further before being digitized by the oscilloscope, as shown in the figure. This arrangement of shallow collector cup and grid with the ion impact angle controlled by the slit has been shown to have no perceptible variation in response over the duration of the measurement (Tiefenback, 1986). Errors associated with the use of 
the slit diagnostics for emittance measurements, along with the linearity of the secondary electron signal are discussed in detail in Chapter 8.

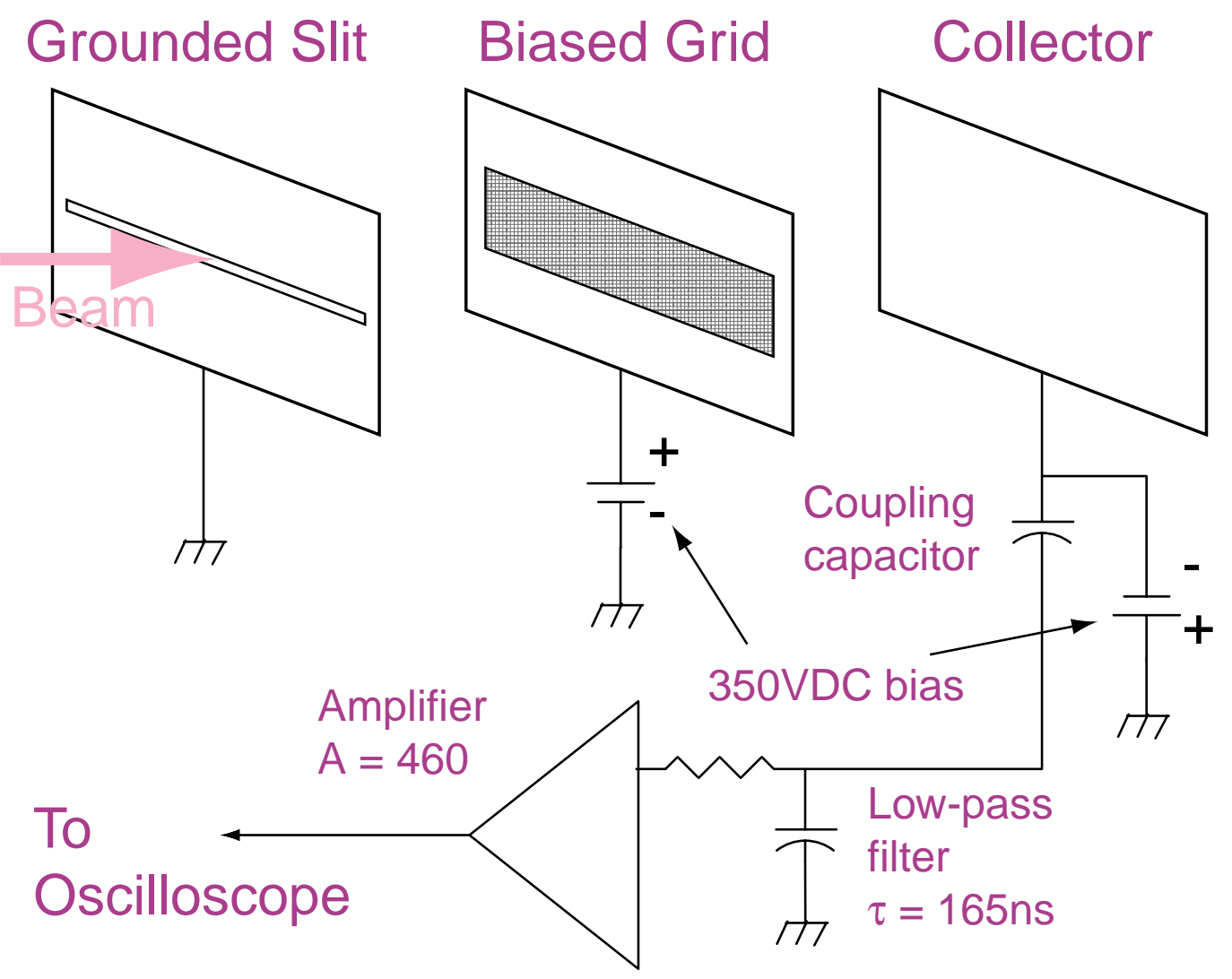

Figure 3.8: Simplified schematic of the slit-cup diagnostic circuitry

By itself, the slit-collector combination ("slit-cup") paddle is used to measure the transverse current density profile of the beam. This measurement integrates over the current density distribution in the plane perpendicular to the motion of the slit. A uniform density beam having a circular cross section projected in this manner produces a cosine-shaped distribution, while a Gaussian density distribution maps into another Gaussian (Chan, 1991). The projection of an axially symmetric distribution on to one dimension is often referred to as an Abel transform. This measurement is typically used to quickly determine the beam centroid and radius in one of the transverse planes. 
Additionally, it is the first indication of a large scale asymmetry or distortion in the transverse current density.

Together, the slit and slit-cup paddles are employed for two different types of measurements as shown in Figure 3.9. Measurements of the phase space density in a transverse plane are performed with the slits aligned in parallel. The upstream slit location determines the position coordinate, and the slit cup is scanned through the transmitted slice to measure the transverse velocity distribution at that position. This procedure is repeated at small intervals (typically $\sim 0.5 \mathrm{~mm}$ in $\mathrm{x}$ and $\sim 0.1 \mathrm{mrad}$ in $\mathrm{x}^{\prime}$ ) across the entire beam distribution to map the phase space density.

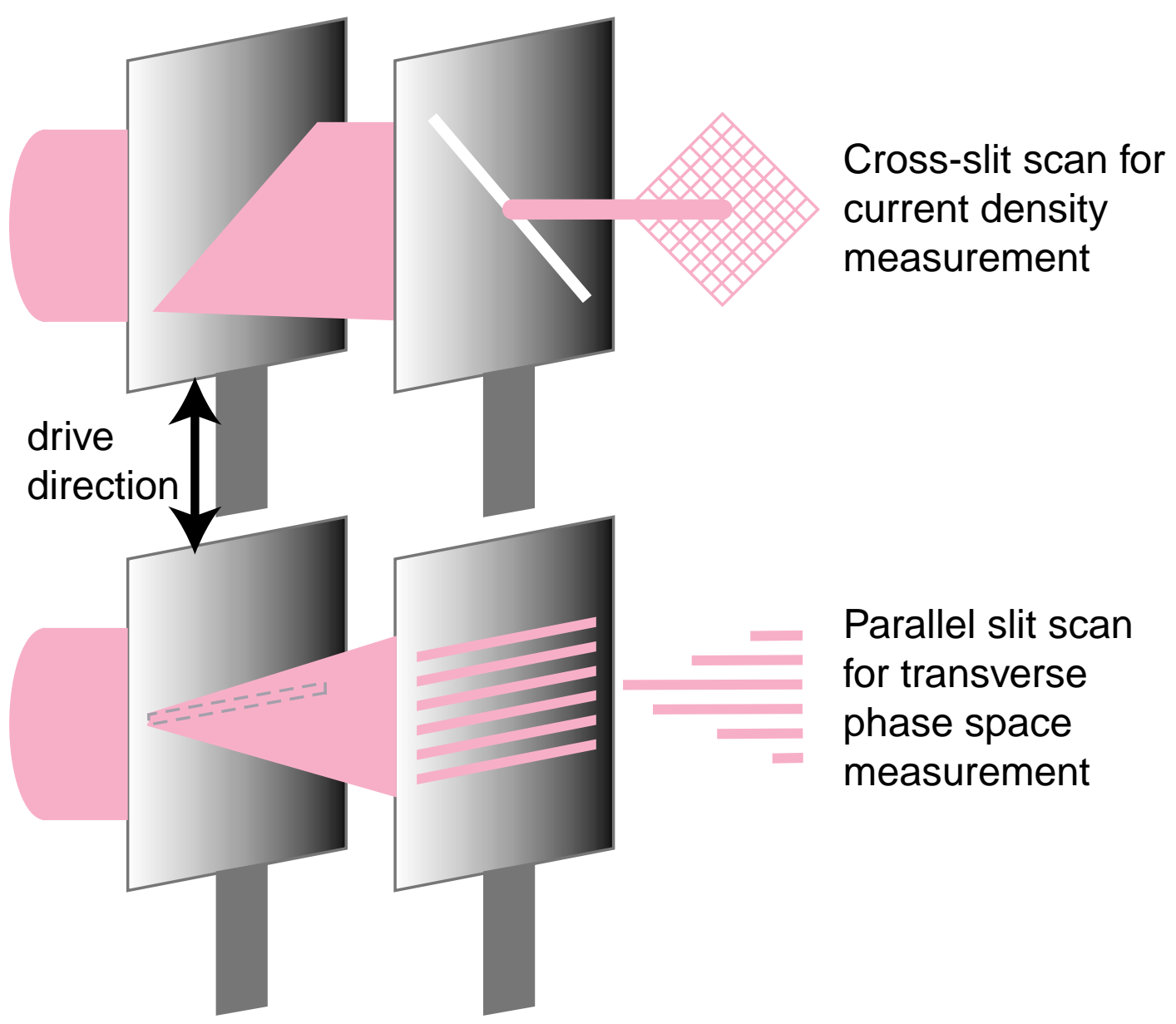

Figure 3.9: Two types of measurements made using slit and slit-cup diagnostic probes 
A slit and slit-cup pair oriented perpendicular to each other is used to map out the current density in the plane perpendicular to the beam motion. Note that this geometry can be created using slits oriented at a $45^{\circ}$ angle to the direction of the paddle motion as shown in Figure 3.9, or with the slit and slit-cup paddles driven in perpendicular directions. The latter is used at stations $\mathrm{D} 3$ and $\mathrm{D} 4$, while the former configuration is used at station D1 where only vertically driven probes are available.

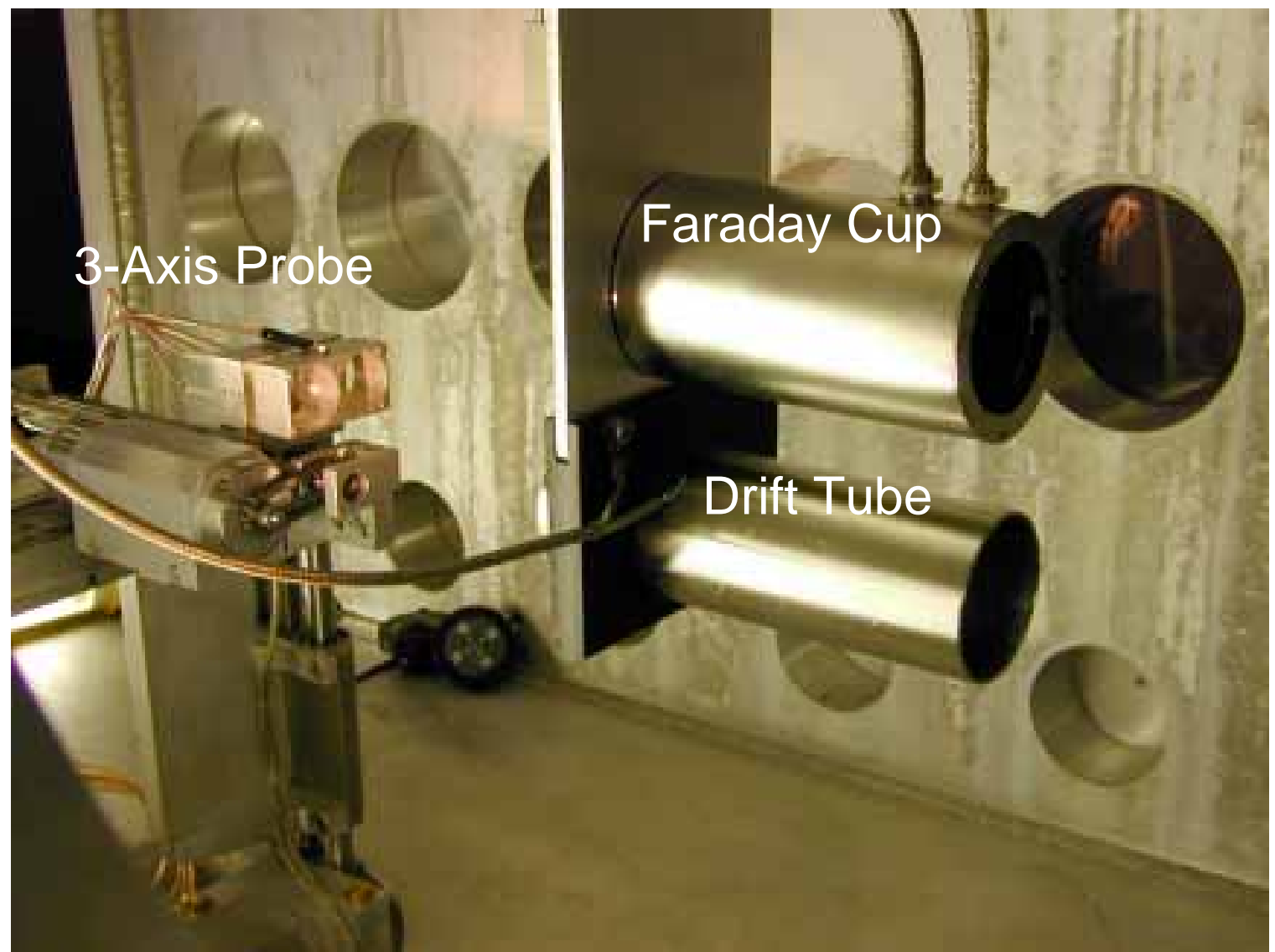

Figure 3.10: Faraday cup (top) and drift tube (bottom) diagnostics in the drift region after the last magnet. To the left is the 3-axis probe used for the focal spot.

Co-located with the deep Faraday cup after the last focusing magnet is a capacitive drift tube diagnostic that is used to measure the total charge of the incident beam (Figure 3.10). As the beam approaches the tube, an image charge collects on the tube. By monitoring the signal on the tube, the current due to the movement of these 
image charges is recorded. Integrating this signal yields a waveform proportional to the charge present in the drift tube as a function of time, shown in Figure 3.11 along with the signal from the co-located Faraday cup. The current spike mentioned at the end of Section 3.1 is clearly present. The degree of neutralization is measured by comparing the signal of an un-neutralized beam to that of a beam containing additional electrons.

Finally, $80 \mathrm{~cm}$ downstream of the last magnet is the focal spot probe, visible to the left of the Faraday cup in Figure 3.10. This probe consists of two shallow slit-cups, each masked with $50 \mu \mathrm{m}$ slits oriented to perform current density scans in both transverse directions. The design of these cups is very similar to those used on the other diagnostic paddles, as it also biased to amplify the ion signal with secondary electrons. The cup assembly is mounted on a platform that translates along all three axes, such that beam spot profiles can be measured at various axial locations within a $15 \mathrm{~cm}$ range. Biased guard rings are mounted in front of the slits in order to confine secondary electrons produced by the incident ions to the slit surface. The rings are necessary to keep the electrons from streaming back up the beam path and partially neutralizing the remaining beam as there are no focusing fields near the final diagnostic probe to deflect them. 


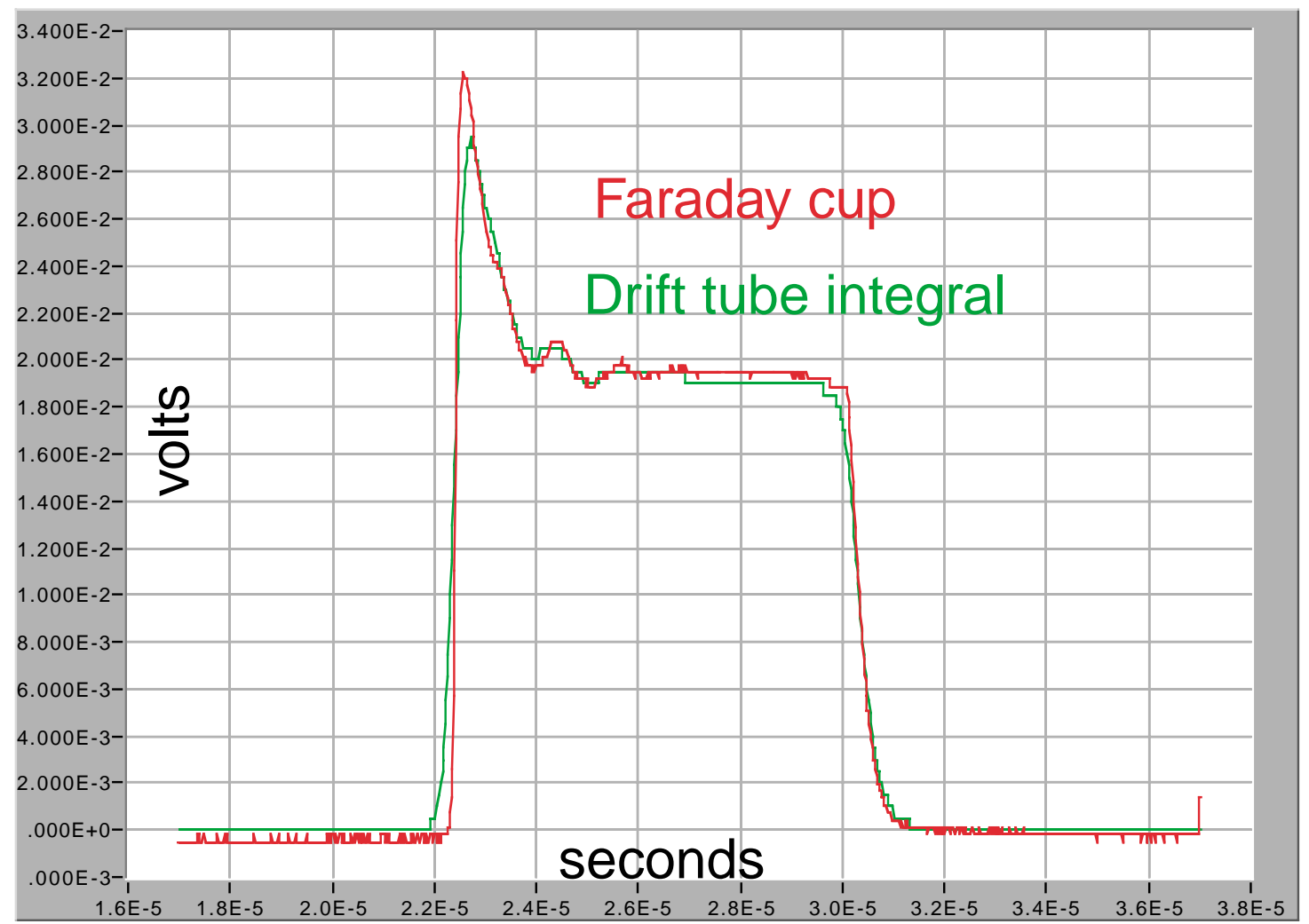

Figure 3.11: Comparison of the integrated signal from the drift tube to the signal from the co-located Faraday cup for an un-neutralized beam. 


\section{Development of the lon Source}

\subsection{Background}

For maximum reliability and pulse-to-pulse repeatability, the current density extracted from the ion source should be maintained at the space charge limit of the diode. To design a less expensive injector with smaller and/or fewer beams, the intrinsic emission limit of the ion source should not prevent the current density of the diode from reaching limits associated with voltage breakdown or beam optics considerations. The other main parameter of concern for the ion source is the emittance. The sum of the intrinsic emittance of the source and the emittance growth due to beam manipulation and transport ultimately determine the minimum focal spot size that can be obtained at the target. Finally, it is desirable to limit the amount of neutral atom emission from the source as the diode optics will be perturbed by charge exchange with the beam ions, and these neutrals can coat and cause breakdown of the nearby high voltage insulators.

A surface ionization source that achieves $>8 \mathrm{~mA} / \mathrm{cm}^{2}$ has been built, tested, and employed in the Scaled Final Focus Experiment. It replaced an aluminosilicate source from a previous experiment that was used as the ion source for preliminary measurements. The source is fabricated by mixing alumina, silica, and potassium carbonate in the proper stoichiometric proportions for $\beta$-eucryptite zeolite, and fusing the material in a vacuum furnace. The product is then ground and applied to the tungsten substrate of the ion source in slurry form. This slurry is again baked in vacuum to form a thin glasslike coating on the substrate (Tiefenback, 1986, p175-176). However, bubbles and other macroscopic non-uniformities would sometimes appear during the second baking that would lead to non-uniform emission. 
In fact, this source produced a beam with a very non-uniform current density distribution and an emittance that was too large for the scaling described in Chapter 2. Based on these results and past experience in the HIF program, it was decided that a surface ionization source would be the best candidate to achieve the uniformity and emittance called for by the scaling. This particular type of surface ionizer, in which Cs atoms are first applied to a refractory metal surface and then evaporated as ions, has routinely produced highly uniform ion beams with low emittance (Seidl, 1998). The resulting transverse emittance for an injector with good optics can be slightly above the lower limit established by the temperature of the emitting surface $(\sim 0.1 \mathrm{eV})$, a value that is difficult to achieve with other source types (Kwan, 1998).

Previous surface ionizers designed as ion thrusters for spacecraft propulsion have used a sintered tungsten plug as a diffuser to control the flow of Cs atoms from a rear mounted vapor Cs reservoir to the thruster's emitting surface (Brewer, 1961). For the

Scaled Final Focus Experiment emitter, the sintered iridium "sponge" is both the contact ionizer and the Cs reservoir; the Cs is stored in an atomic state on the large surface area within the porous iridium volume. Based on the work function of iridium and the observed lifetime of the source, it is suspected that surface diffusion and evaporation of neutral atoms control the depletion process.

\subsection{Mechanical Design}

In the experiment, the source assembly is fit into the back of a $13.3 \mathrm{~cm}$ diameter graphite anode. The graphite is machined to produce a conical angle of $22.5^{\circ}$ with respect to the surface of the emitter. This angle, often referred to as a Pierce angle, creates a potential at the edge of the extracted beam that approximates that of an infinite 
beam in order to produce a laminar beam with uniform current density (Pierce, 1949, Humphries, 1990, pp. 262-277). The A grounded cathode with an aperture to extract the beam is located $5 \mathrm{~cm}$ in front of the emitting surface, and the entrance to the first electrostatic quadrupole is $6.6 \mathrm{~cm}$ from the downstream face of the cathode. A cutaway view of the source, diode, and first electrostatic quadrupole inside the vacuum tank is shown in Figure 4.1. Supporting the diode is the mechanism that allows for steering the source from outside the vacuum tank.

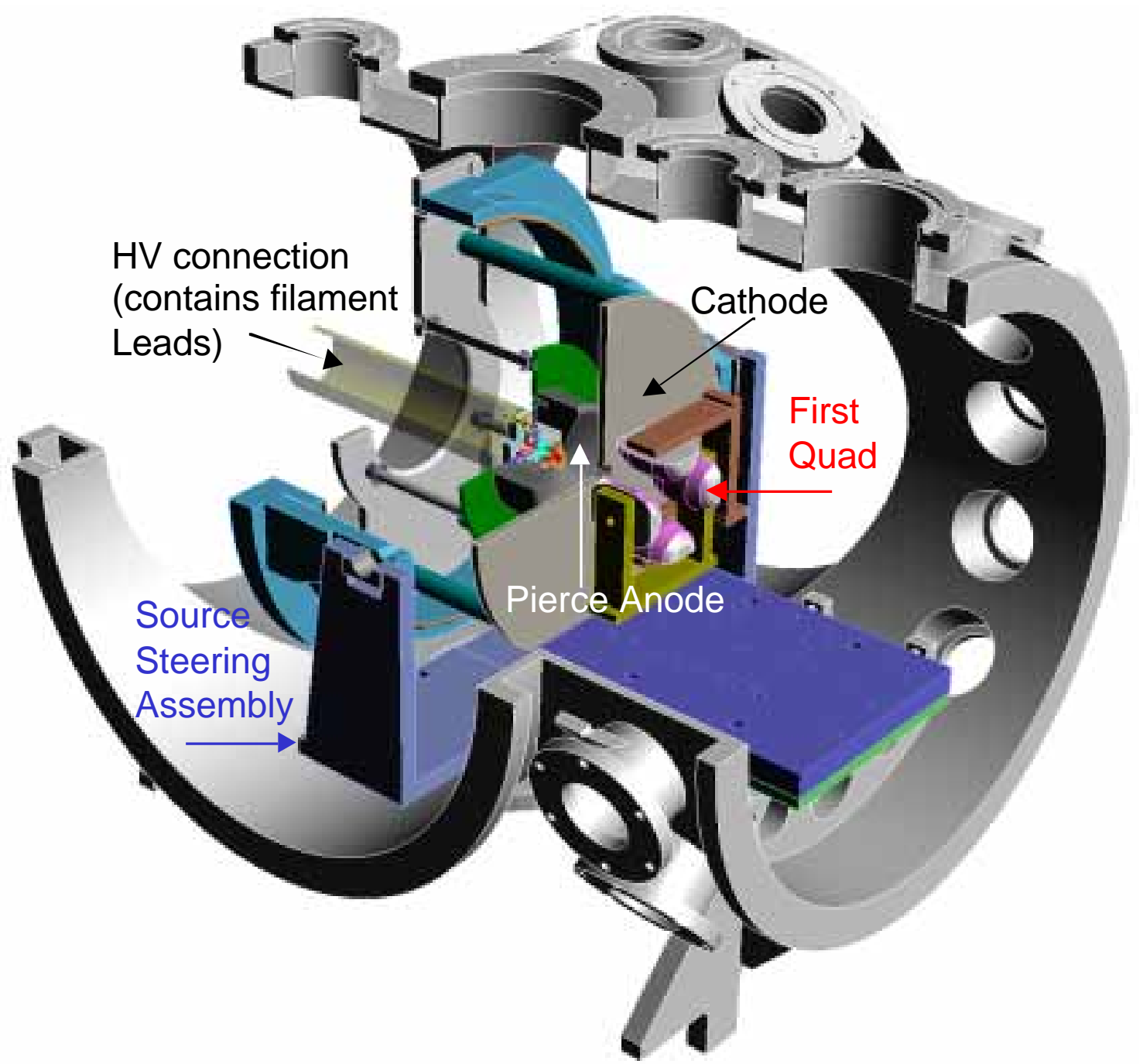

Figure 4.1: Cutaway view of the source enclosure and first electrostatic quadrupole 


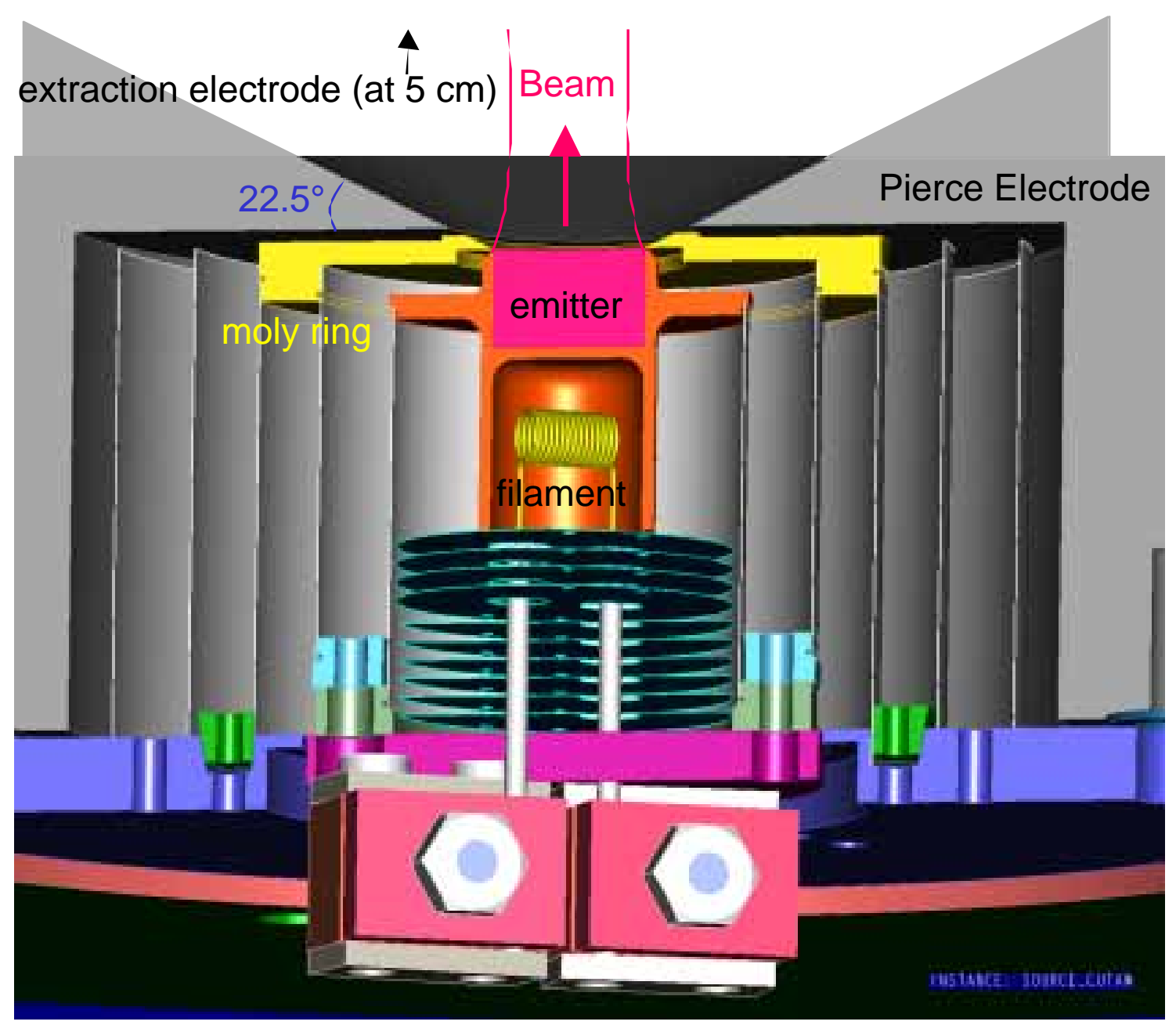

Figure 4.2: Cross section view of the ion source

Figure 4.2 shows a cutaway view of the complete source, including the sintered iridium emitter, the filament, the heat shields, and the graphite Pierce anode. Figure 4.3 is a photograph of the assembly, excluding the graphite anode, looking down from the top. The heat shield assembly was built with an emphasis on achieving higher emitter temperatures $\left(>1150{ }^{\circ} \mathrm{C}\right)$ for higher current densities with an iridium emitter while still maintaining good thermal efficiency. In contrast to previous designs that placed the filament in contact with ceramic insulators, this filament is freestanding, allowing for higher filament temperatures and longer lifetime. To date, the filament has operated with the source between 1000 and $1100^{\circ} \mathrm{C}$ for $\sim 2000$ hours. The path for conductive heat 
transfer follows a reentrant route from the molybdenum cup holding the emitter, through three concentric thin $(0.005 ")$ molybdenum cylinders, to the back plate of the assembly which acts as a thermal sink. Radiation to the sides and rear of the filament encounters many layers of highly reflective shielding.

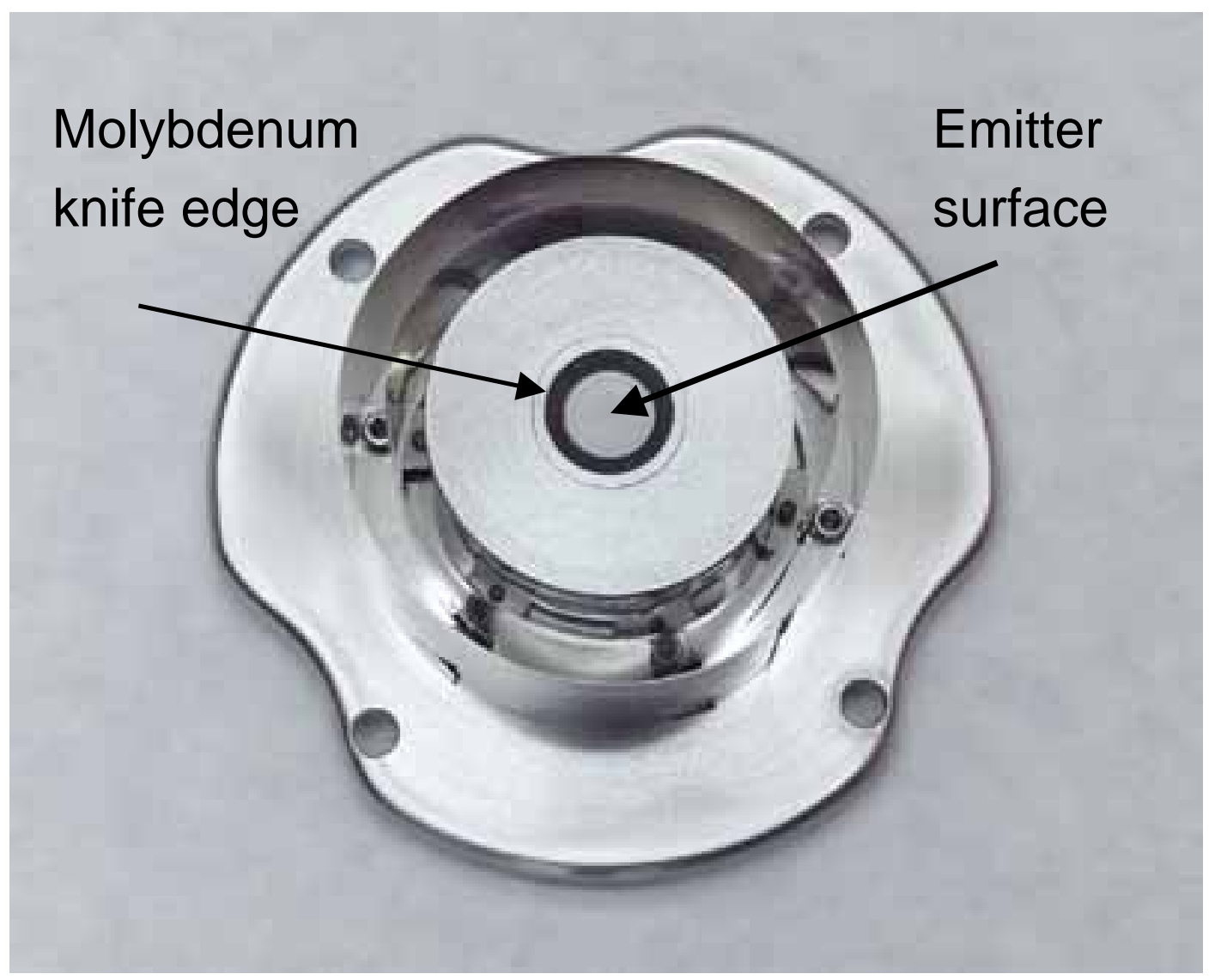

Figure 4.3: Ion source and heat shield assembly

The curved (11 cm radius) emitting surface is the end of a sintered iridium cylinder $1 \mathrm{~cm}$ in diameter and $1 \mathrm{~cm}$ long. This emitter was finished to size with a diamond grinder, including grinding the spherical surface. This surface was then etched using electric discharge machining to recover the porous nature of the sintered material at the emitting surface. This allows Cs atoms attached to the internal surfaces of the sintered metal to migrate forward and repopulate the emitting region. Figure 4.4 shows an electron microscope image of the finished porous emitting surface, where a $10 \mu \mathrm{m}$ 


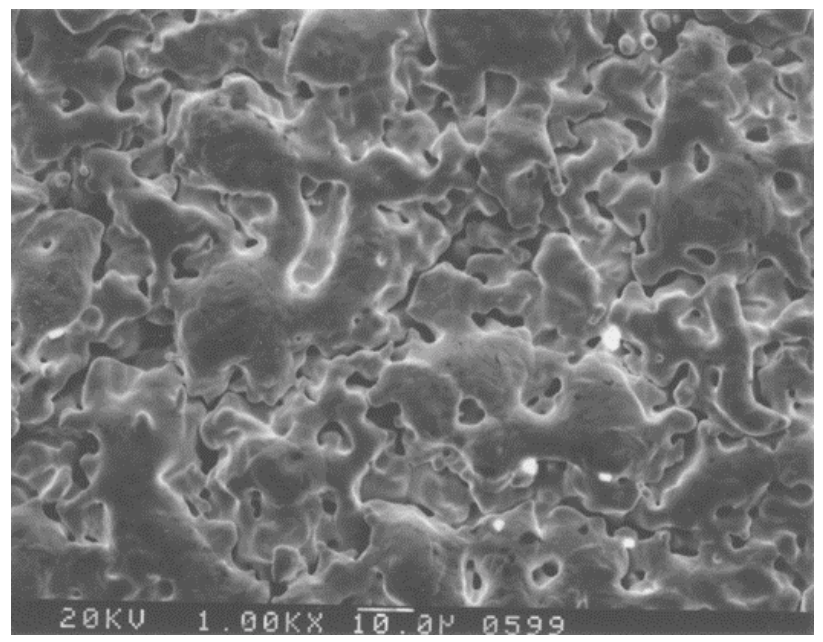

Figure 4.4: Electron microscope image of the surface of the emitter. A $10 \mu \mathrm{m}$ line at the bottom of the photograph indicates the scale of the features. dimension is indicated at the bottom of the image. The size of the typical structure is small so as to be negligible for the ion optics compared to the transverse thermal velocity distribution. The graphite electrode forms the bulk of the anode geometry, but the sharpness of the inner edge is limited by the brittle nature of the material.

Previous experiments have shown that the properties of the extracted ion beam are very sensitive to the geometry in the immediate vicinity of the emitting surface. Therefore, a molybdenum intermediate ring is used so that the inner surface could be machined to a "knife edge", starting the Pierce angle of the electrode as close as possible to the emitter edge. The less precise edge of the graphite is thereby moved away from the critical region adjacent to the emitter. It is positioned within $125 \mu \mathrm{m}$ of the emitting surface but does not make mechanical contact.

\subsection{Contact lonization and Lifetime}

Sintered tungsten emitters of the type described above have been used as contact ionizers to obtain emission currents of $\mathrm{J} \geq 2 \mathrm{~mA} / \mathrm{cm}^{2}$ (Celata, 1996, Kwan, 1998). The change to iridium was made with the expectation of reduced Cs neutral emission. From the Saha-Langmiur equation, the ion to neutral emission fraction from a clean surface is (Forrester 1988): 


$$
\frac{v_{i}}{v_{n}}=2 \exp \left[\frac{(\phi-I)}{k T}\right]
$$

where $\phi$ is the work function of the metal and I is the ionization potential of the adsorbed atom. The work function for tungsten is $4.6 \mathrm{eV}$ and for iridium is $5.7 \mathrm{eV}$, resulting in ratios of $\sim 10^{3}$ and $\sim 10^{6}$, respectively for a coating of $\mathrm{Cs}$ at $1100^{\circ} \mathrm{C}$. While it is not expected that clean surfaces exist in the vacuum system at $10^{-6}$ torr, iridium, being a noble metal, is fairly clean at the $1000-1200{ }^{\circ} \mathrm{C}$ operating temperatures. The above estimates are therefore representative of the expected emission differences between $\mathrm{W}$ and Ir. The duty factor of an HIF injector is $2 \times 10^{-4}$, so neutral emission would dominate the depletion of $\mathrm{Cs}$ from tungsten, but not necessarily from iridium. This ionization rate also implies that the overall emission is somewhat less for iridium than for tungsten, so the sintered iridium source will have to operate at a higher temperature to achieve the same ion current density.

For a given temperature, the peak value of the contact ionization current density typically occurs at a very low surface coverage fraction. This is evident in Figure 4.5, which also shows a typical operating path for a source following re-supply. The data for the figure come from empirically determined coefficients for cesium on tungsten at $1075^{\circ} \mathrm{C}$ (Taylor, 1933). For an initial surface coverage of $\theta=3 \%$, the current density is below the space charge-limited value of $8 \mathrm{~mA} / \mathrm{cm}^{2}$ at $160 \mathrm{kV}$ extraction voltage. Holding all other parameters constant, the coverage fraction is allowed to decrease due to neutral emission until the current density has reached the space charge limit. The "lifetime" of the source is typically defined as the time during which the source will operate at the space charge limit (horizontal red arrow). The source is considered depleted when the 
coverage fraction is too low to support space charge limited emission and the total current produced begins to decrease with time.

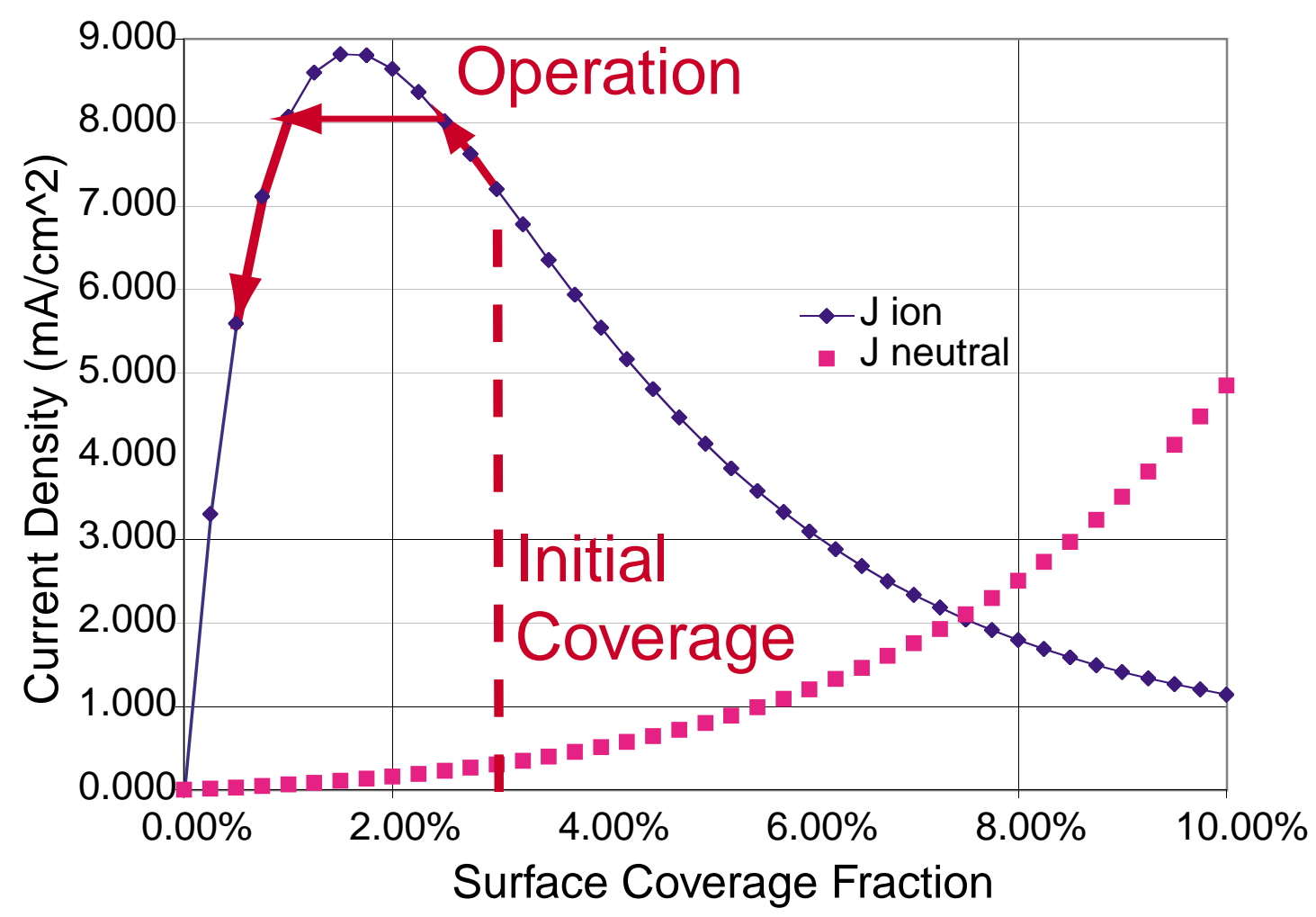

Figure 4.5: Current density versus coverage fraction for $\mathrm{Cs}$ on $\mathrm{W}$ at $1075^{\circ} \mathrm{C}$

On the tungsten surface, the diffusion models developed by Taylor and Langmuir assume a characteristic time of $\sim 1$ ps for a cesium atom to "hop" from one site on a tungsten substrate to the next (Taylor, 1933). We can therefore estimate a diffusion time of $\tau \approx 100$ seconds for a uniform Cs coverage of a centimeter-scale tungsten emitter compared to an observed emission lifetime of $\sim 20$ hours, so at any instant the surface coverage $\theta$ is constant within the sponge. For the iridium emitter, where Cs atoms are more tightly bound to the surface, there is some indication that there exists a non-uniform surface concentration within the volume of the cylinder. The diffusion equation predicts that the distance a particle will travel, say from the rear of the iridium cylinder to the emitting surface, is proportional to $\mathrm{t}^{1 / 2}$. During depletion the data have shown this $\mathrm{t}^{1 / 2}$ 
time dependence, as illustrated in Figure 4.6. In the iridium sponge, if diffusion from the volume of the sponge to the emitting surface is the limiting process, we should be able to significantly increase the surface coverage in the interior region by supplying the source initially with more Cs, increasing the effective lifetime of the source.

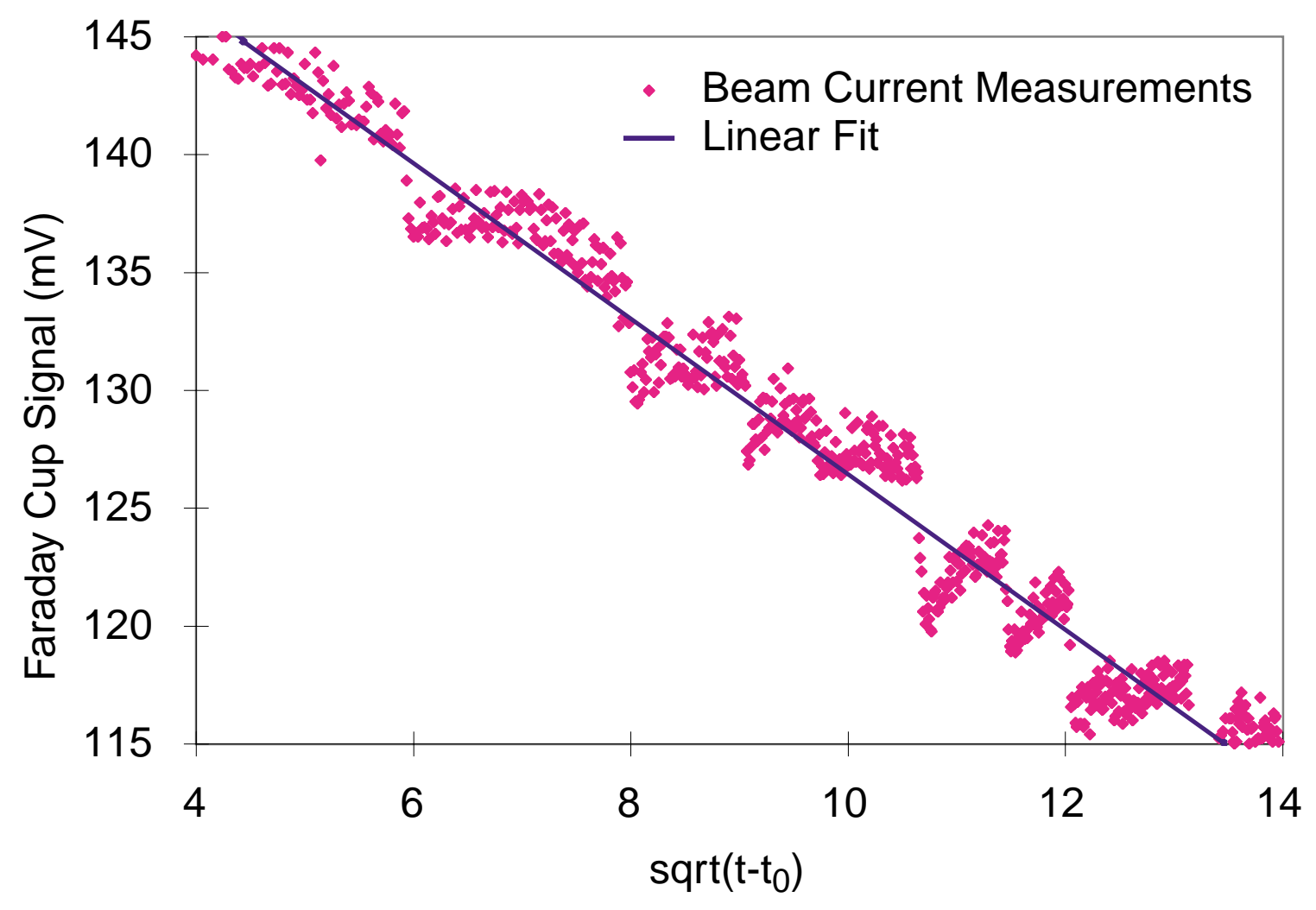

Figure 4.6: Cesium ion emission versus $\mathrm{t}^{1 / 2}$ during depletion of the iridium source

\subsection{Performance}

The thermal performance of the heat shield assembly is quite good. During initial testing, the emitter surface was raised to a temperature of $1140^{\circ} \mathrm{C}$ using a filament power of 40 watts, and the source was operated at an emitter temperature as high as $1225^{\circ} \mathrm{C}$ using 52 watts. Upon disassembly, there was no visible degradation of the filament or damage to any of the heat shield package components. 
Measured over many re-supply periods, the lifetime of the $0.8 \mathrm{~cm}^{3}$ iridium source averages 45 hours at the operating temperature of $1100-1150{ }^{\circ} \mathrm{C}$. This compares favorably to the 15 hour lifetime of tungsten sources with the same volume. Additionally, the iridium source is operated at a current density of $8 \mathrm{~mA} / \mathrm{cm}^{2}$ compared to the 2 $\mathrm{mA} / \mathrm{cm}^{2}$ operating point for the tungsten sources. The ratio of extraction time to total time at temperature (duty factor) is approximately $10^{-6}$ for both sources.

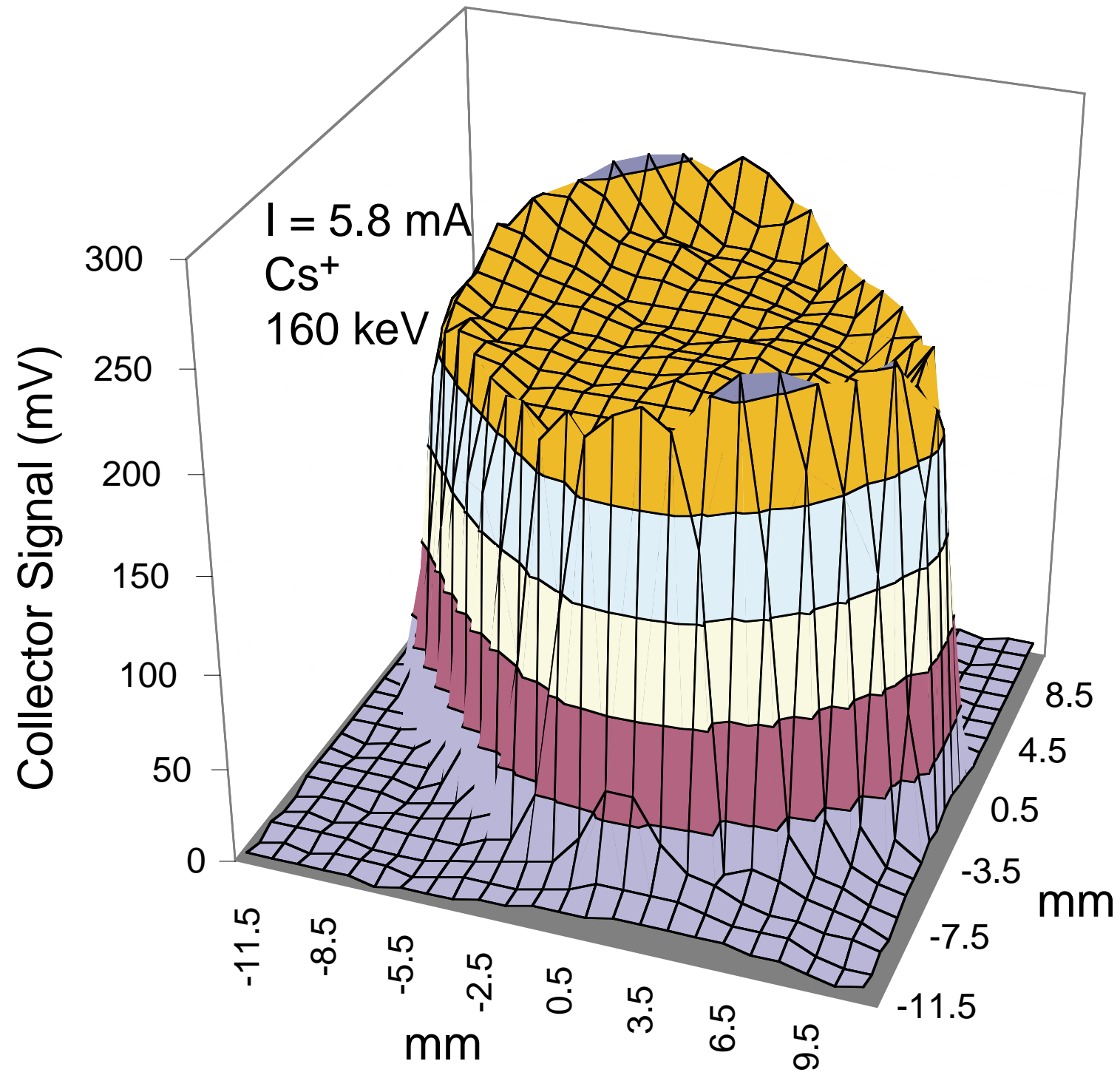

Figure 4.7: Transverse density of the full current from the iridium source 
Figure 4.7 shows a current density map of the full beam after extraction from the source and transport through the first five electrostatic quads in the experiment. For the scaled final focus, a $1 \mathrm{~mm}$ diameter aperture intercepts the beam, passing only the core of the beam to the rest of the experiment. The beam displays a very uniform profile with some distortion at the edges as a result of filling a large fraction of the quadrupole aperture during transport to this point. Figure 4.8 is a phase space density map of the vertical transverse plane $\left(Y-Y^{\prime}\right)$ at the same location. From this type of measurement, the value of the normalized edge emittance of $1.8 \times 10^{-8} \pi$ meter radians is obtained. The theoretical thermal emittance for the source, calculated from Equation 2-21, is $1.0 \times 10^{-8}$ $\pi$ meter radians.

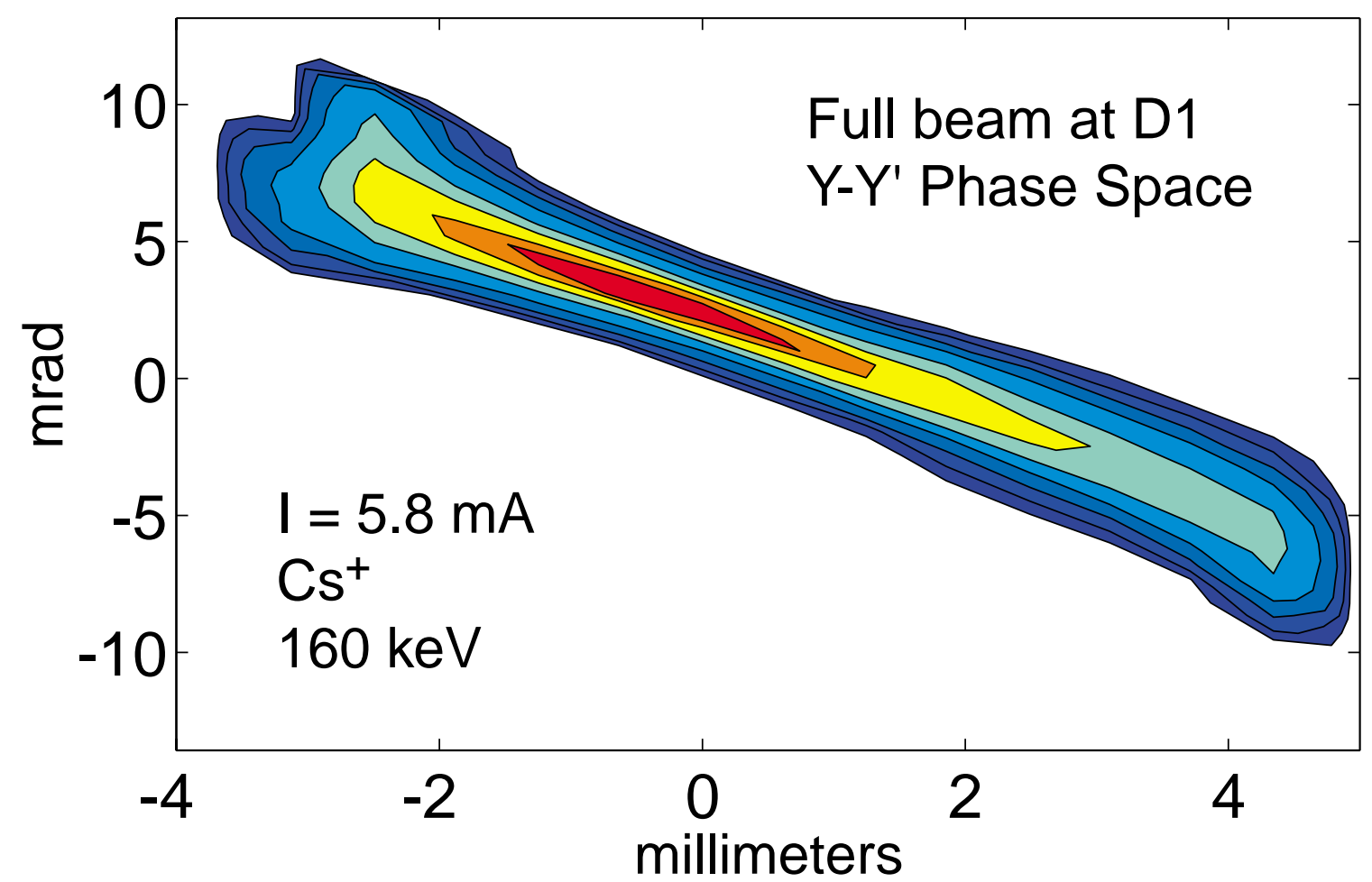

Figure 4.8: Transverse phase space of the full current from the iridium source For any charged particle beam, the beam brightness is a measure of the overall quality of the beam and is typically defined as the current density per unit solid angle in 
the axial direction. It is proportional to the ratio of the beam current to the product of the transverse emittances (Humphries, 1990, p. 105-6). The aluminosilicate source mentioned above delivered a current density of $2.1 \mathrm{~mA} / \mathrm{cm}^{2}$ and a normalized edge emittance of $4.4 \times 10^{-8} \pi$ meter radians. The iridium surface ionization source therefore represents a factor of three improvement in the brightness of the extracted ion beam. 


\section{Experimental Methods}

\subsection{Data Acquisition System}

The slit and slit cup paddle pairs described in Section 3.4 serve to collect the majority of the data for the experiment. The data acquisition process associated with these diagnostics involves triggering the Marx generator to produce the beam, collecting the signal that is produced by the portion of the beam incident on the shallow Faraday cup collector, and positioning the paddles for the next data point. The pulse from the collector is digitized and stored initially on an oscilloscope, and then immediately downloaded to a computer. This process is repeated approximately 200 times to perform a complete $x$ or $y$ phase space scan of the beam, or a map of the current density ( $x$ vs. $y$ ).

As it is desirable to perform these scans frequently to assess the effects of any manipulation of the experimental apparatus, it is necessary to fully automate the process in order to obtain results in an efficient manner. A complete data acquisition and control software package is written in LabView ${ }^{\mathrm{TM}}$, a graphical programming language from the National Instruments Corporation. This code is used to define the measurement boundaries, perform the sequence described in the paragraph above, and store the data to a file with a format suitable for further analysis. Step motors are used to position the slits with a $10 \mu \mathrm{m}$ accuracy. The software uses a General Purpose Interface Bus (IEEE Standard 488) interface to communicate with the step motor driver and the oscilloscope. An analog output from a multifunction I/O card is used as the trigger signal for the experiment.

The software is divided into five main modules. The Move module is used to manually position individual drives to test for an expected signal, and to house the drives 
after a measurement has been performed. The Phase module uses a graphical user interface to manually map out transverse phase space and determine suitable measurement boundaries for an automated scan. The user places the cursor at a location in the $x-x^{\prime}$ or $y-y^{\prime}$ plane, and the system moves the paddles, triggers the pulser, and collects and displays the resulting signal amplitude. The user can then draw a boundary in phase space for an automated measurement, as is shown in Figure 5-1. The Scan module uses this boundary information to perform the measurement. The Profile module performs the single slit scans of the beam, and the Map module performs the cross slit scans. Both of these measurements are set up using user specified boundaries and step sizes.

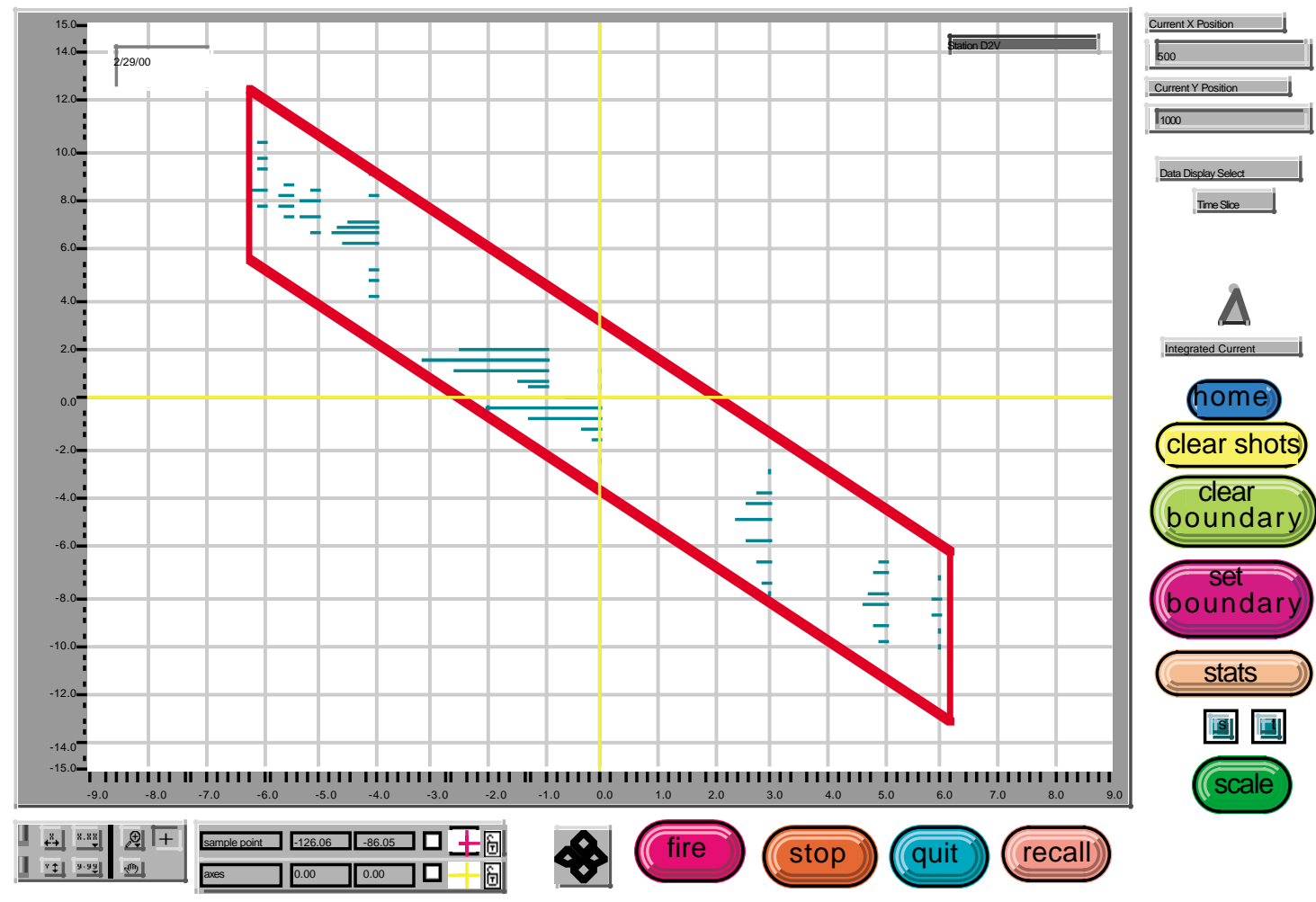

Figure 5.1: Phase program interface for sampling phase space and setting up the measurement boundary for an automated scan

The Profile module is also used in conjunction with the 3 -axis probe that measures the focal spot. The Move module can be used to position the slit cup in the 
axial direction, and center it in the transverse direction perpendicular to the direction of the scan. Profile scans can then be performed at a series of axial location to determine the location of the beam waist in either of the transverse planes.

\subsection{Use of the Envelope Code}

As mentioned in Section 3.4, the portions of the experiment between the diagnostic stations can be modeled with a simple code that calculates the beam envelope parameters as a function of axial position (De Hoon, Fawley, and Novotny). The program numerically integrates the envelope equation through a set of external focusing fields described by an input file. The code is written in the Java programming language, and employs a graphical interface that allows the user access to all of the experimental parameters that can affect the terms in the envelope equation. As will be described below, the relative simplicity of the calculation combined with the ease of use of the graphical interface make the code a convenient tool to determine the focusing gradients needed for the desired final envelope conditions, and also for a rapid first analysis of experimental measurements.

The semi-major and semi-minor radii and convergence angles of the beam edge in both transverse planes are the four parameters that completely describe the beam envelope at any one axial location. In the basic mode, the code integrates these parameters through a user specified set of focusing strengths using a fourth order RungeKutta method. The user may adjust the strength of any of the elements through a continuous range with a slider style graphical interface. The code can also be run in a solver mode when there are four unspecified strengths for a particular set of focusing elements. This function inverts the matrix that transforms the beam parameters from 
initial to final conditions, comparing calculated parameters from the iteration to desired parameters as the convergence criteria. A combination of these modes is typically useful in the electrostatic section where there are five elements before each diagnostic station. In this case, the maximum envelope excursion can be adjusted by manually tuning the strength of the first element and allowing the solver to find the strengths of the last four elements that produce the desired final beam parameters.

Prior to making an initial measurement of the beam at any of the diagnostic locations, the code is typically employed as discussed above to calculate the strengths of the focusing elements necessary to transport the beam to the next diagnostic station. Initial envelope conditions that have been either measured or calculated are specified as inputs along with the lengths of the focusing elements and drift spaces. Since the field in the code is linear, the focusing gradient is specified through a fixed aperture radius and an electrode voltage. For the magnetic elements, the field at the aperture radius is converted to an equivalent electrode voltage at the beam energy for input into the code. The values obtained from the code are then set into the experiment and the beam parameters are measured.

Important virtues of the code are its user interface and its portability. These allow for its use as a tool for immediate feedback while the experiment is running. Once the beam envelope parameters are obtained from measurements, the code is used to iterate the original solution in order to get better agreement between the measured parameters and the desired final conditions. The initial disagreement is primarily due to approximations used in the calculation of the magnetic field gradient from the peak pulser current, but other sources of error arise from the small uncertainties in the beam 
current and emittance. For example, a measurement made at D3 is compared to the solution produced in the code to achieve the desired parameters from the initial conditions measured at D2. The strengths of the first three magnets are manually adjusted in the code until the final parameters more closely match what was measured, to obtain a fit for the existing magnet strengths. The fit values are used with the original values to calculate corrections that can be applied to the magnet pulser settings.

\subsection{Presentation of the Data}

Throughout the length of the experiment, two types of measurements are typically made with the two slit diagnostic pairs and the data recorded for further analysis. The most common measurement is the phase space scan, where the beam amplitude is measured as a function of both position and transverse momentum along $x$ or $y$. The data is time resolved; however, a section near the center of the pulse is usually selected and integrated over for the analysis. First and second moments are extracted from this data, yielding the centroid position and angle of the beam as well as the extent of the beam edge in transverse position and momentum. Additionally, the moments are used in the calculation of the beam emittance as described in Chapter 2 .

An analysis routine written in MATLAB ${ }^{\mathrm{TM}}$ (a computation and visualization program from the MathWorks corporation) performs the above manipulations for many sets of phase space data. The entire data set is integrated over all of phase space and plotted as a function of time to determine the steady state region; usually, a time interval can be defined where the integrated signal varies by only $\sim 1 \%$. One important factor in any of the calculations is the amount of phase space area included. When the slits are in a position during the scan where there is no beam signal present, a certain level of noise 
is collected by the diagnostic. If this noise is particularly far from the centroid of the data, it can weigh heavily into the calculation of the second moments. It is therefore useful to be able to exclude from the calculation a known fraction of the data whose amplitude lies below a given threshold. Without background noise, a plot of calculated emittance versus percent signal included would produce a smooth function to $100 \%$. This type of plot is shown in Figure 5-2, indicating the sharp break in the slope of the data due to the addition of the noise. The emittance measurements and phase space plots presented in this paper are based on a cut made in the manner of Figure 5-2, with the signal included in the calculation typically $\sim 95 \%$ of the total collected during the scan.

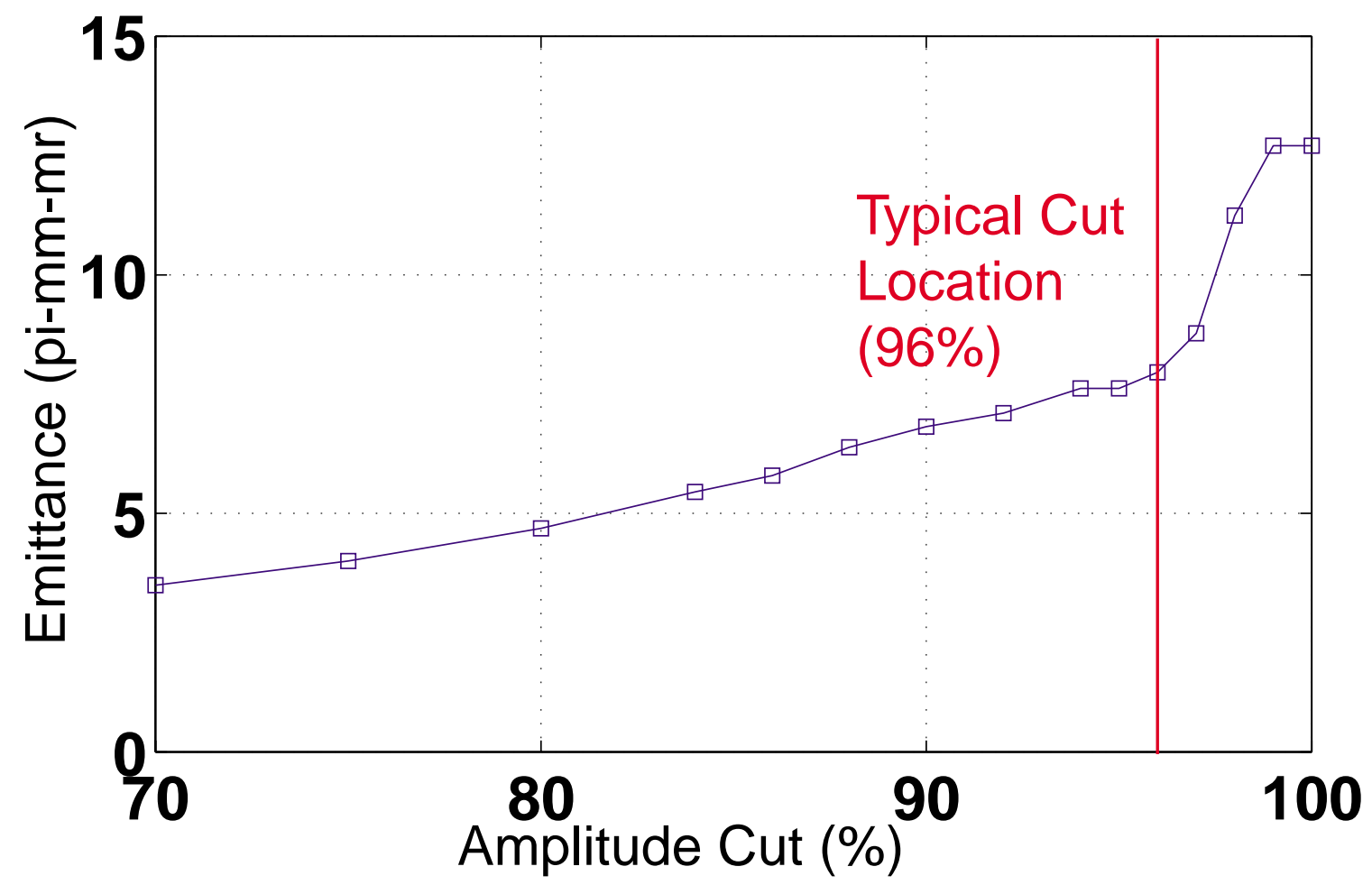

Figure 5.2: Plot of measured emittance versus percent of total signal for a phase space measurement at D4. The red line indicates where the emittance would be evaluated.

Once the cut described above is made, there are other errors that can enter into the experimental determination of the emittance. Wider slits are used at D3 to ensure that there is sufficient signal amplitude as the beam semi-major and minor axes are at their 
largest. This leads to an artificial enhancement in the transverse velocity distribution and therefore the calculated emittance. This and other sources of error are described in detail in Chapter 8.

The phase space measurements are typically studied in contour plot form. Contours representing various levels of signal amplitude are drawn around the data plotted in the plane of transverse phase space appropriate to the measurement. Aberrations in the beam are produced by deviations from linear transverse forces and therefore are proportional to higher powers of the radius. Due to this dependence, they are manifest as distortions to the edge of the beam or the "ends" of the phase space distribution. To aid in the study of these aberrations, it is helpful to implement a coordinate transformation that zeroes the cross moment of the distribution. This is accomplished by subtracting the quantity $\frac{\left\langle x x^{\prime}\right\rangle}{\left\langle x^{2}\right\rangle} x$ from the $x^{\prime}$ coordinate of each data point. The net effect is to render a plot in which the vertical axis distribution highlights the nature of the distortions to the ends of the beam while preserving the position $(x$ coordinate) of each data point.

The other type of measurement that is routinely made at each of the first four diagnostic stations is the crossed-slit scan, using two orthogonal $1 \mathrm{~mm}$ wide slits to produce a transverse current-density map of the beam. This type of data is most clearly displayed in a surface plot format with the height of the surface corresponding to the signal produced by the incident beam current at the corresponding location in the transverse plane. This data is also time resolved, and the analysis routine that is used to examine the crossed slit data files can produce a plot corresponding to a particular time "slice" in the beam pulse. In addition, the routine can "animate" the data, running 
through the time slices in succession in order to illustrate time dependent behavior of the current density.

The measurements of the beam focal spot are performed by the three axis diagnostic at D5. The slit cups on this probe perform single slit scans that generate profiles of the beam spot in both of the transverse planes. The profile data is typically examined in a routine that handles the time resolution frame-by-frame as in the crossed slit analysis program. A mean and an rms width are calculated and displayed for each time slice, and the profile data at each time can be fit with a Gaussian function or a sum of two Gaussians. Additionally, the fraction of included beam current as a function of transverse position can be plotted. This particular type of plot is particularly useful in determining the quality of the focus, as it ultimately relates to the energy that will be provided to the target to drive the fusion reaction. Finally, the rms widths can be plotted as a function of time, and a surface plot of the complete data set showing signal amplitude as a function of time and position can be generated.

\subsection{Comparisons with the WARP Simulation}

WARP, a particle in cell code, is used to model the scaled final focus experiment in both the $95 \mu \mathrm{A}$ and $400 \mu \mathrm{A}$ configurations. The simulation of the experiment used the 2D transverse slice version of the code (Grote, 1998). The code employs a 256 x 256 grid of cells with four-fold symmetry containing 40,000 macroparticles in one transverse slice. The slice propagates through the applied fields and feels the effects of forces in the transverse direction only.

The focusing fields are modeled as pure quadrupole fields. For the $400 \mu \mathrm{A}$ simulation, the focusing gradients were extended beyond the edge of the physical 
quadrupoles, decreasing smoothly to zero in a length equal to the aperture radius for both electrostatic and magnetic elements (DeHoon, 2000). This includes in the dynamics of the pseudo-octupole component that arises from the axial gradient of the field. The grid size is adjusted according to the beam size, increasing in the electrostatic section, and reducing proportionally to the beam radius as the beam is focused to a spot. The location of the conducting surfaces near the beam in the experiment was accurately represented so that the fields due to the image of the beam's space charge would appear correctly in the simulation. In particular, the electrostatic quadrupoles are modeled as four conducting half-cylinders, and the conducting foil inserted in the magnets is represented by a conducting boundary at the magnet aperture radius.

In order to facilitate comparisons of the measurements with the output of the simulation, calculations within the code are made at the same axial locations as the experiment's diagnostic probes. The output, in the form of coordinates of the macroparticles, is converted to amplitudes by binning the particles in phase space with the same spacing and dimensions as the sampling intervals and measurement boundaries in the experimental measurement. With the data from the WARP run in this format, the same MATLAB ${ }^{\mathrm{TM}}$ analysis routine is used to display the phase space as a contour plot and extract important parameters. 


\section{6. $95 \mu \mathrm{A}$ Results}

\subsection{Data Before The Focus}

Table 2-1 identifies $95 \mu \mathrm{A}$ as the current for the experimental beam that will match the perveance of the HIBALL-II beam in the final focus system. In this section, measurements of the $95 \mu \mathrm{A}$ beam as it traverses the focusing lattice on its way to the focal spot are presented in the form of transverse phase space and current density plots. Additionally, phase space output from the WARP model described in the previous section is presented for comparison with the data at the various diagnostic locations. Side by side, these comparisons are used to help interpret the causes of the phase space distortions and changes in emittance observed in the experiment.

The envelope for the $95 \mu \mathrm{A}$ beam is shown in Figure 6.1. At the top of the figure is the to-scale schematic of the experimental hardware, as in Figure 3.2, showing the locations of the focusing elements. Below, the corresponding colored bars indicate the quadrupole apertures. The blue curve represents the vertical envelope (twice rms radius) of the beam, and the red curve the horizontal, with the diagnostic locations indicated by the dashed lines. For the experimental measurements, the data presented in this section is time integrated over a 2-3 $\mu$ s interval during the middle of the pulse, and the phase space distributions are sheared using the method described in Section 5.3. The emittance is calculated as a function of percent of the data included. A "knee" in this curve indicates the point the background noise causes the emittance to deviate significantly from the curve expected in the absence of noise. As remarked in Section 5.3, this cutoff often occurs at about $5 \%$ of the data. 


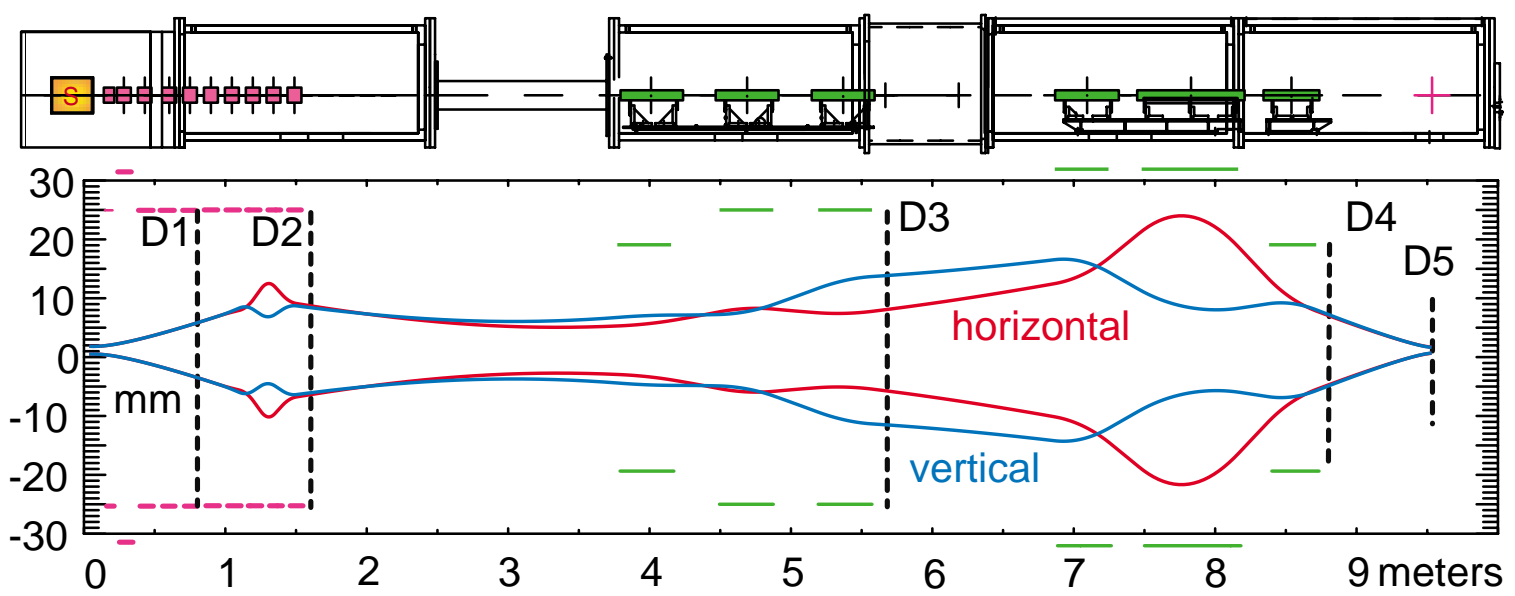

Figure 6.1: Horizontal and Vertical envelopes for the $95 \mu \mathrm{A}$ beam

The remaining $95 \%$ is plotted as a function of intensity, the contour levels representing $2 \%, 5 \%, 10 \%, 20 \%, 40 \%, 60 \%, 80 \%$, and $90 \%$ of the data that remain after the cut. On the other hand, the WARP figures are derived from particle dumps during the run and therefore contain $100 \%$ of the data. Features at the edge of the beam involving low particle density will therefore be detailed more clearly by the WARP plots. The phase space distribution in the vertical plane at the first station, D1, is represented at the top of Figure 6.2 by a contour plot of the measured intensity. Below, the vertical phase space from the WARP simulation for the same location is shown. In this first figure, a color bar indicates the fractional intensity levels represented in the plots. Quoted above each plot are the four times rms emittances $\left(4 \varepsilon_{\mathrm{rms}}\right)$ calculated from each set of data.

The vertical phase space at the next diagnostic station, D2, is presented in Figure 6.3, again with the simulation below the measurement for comparison. Notice that in both cases the emittance has increased, and in the second measurement, distortions are beginning to develop similar to those present in the simulation. From Figure 6.1, it is apparent that the beam radius has increased by a factor of 20 during its transport through the electrostatic quadrupoles. In the WARP run, the S-shaped appearance is believed to 
be due to the non-linear space charge force as the beam expands in this section (De Hoon, 2000).
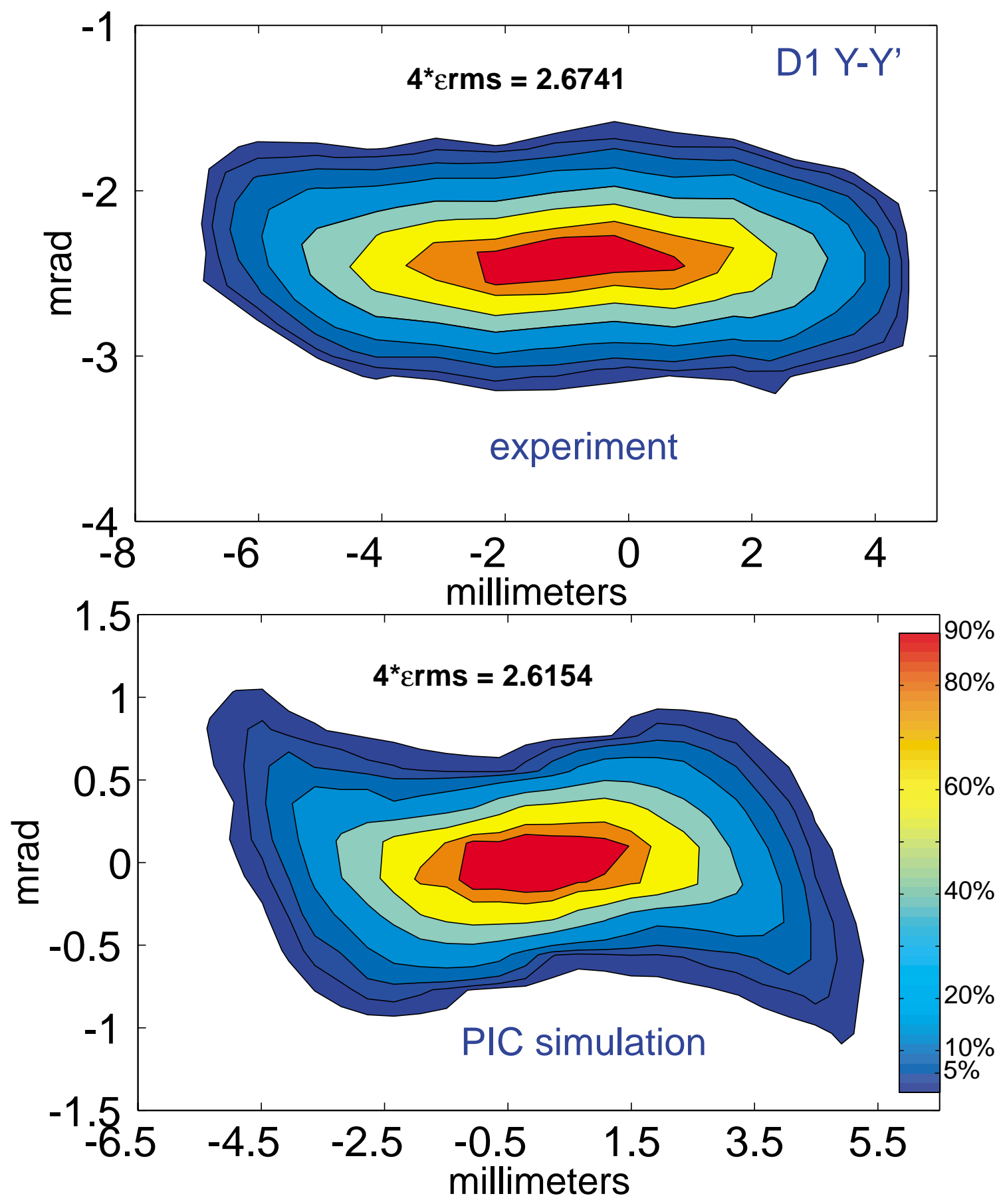

Figure 6.2: Transverse phase space at D1; measured and from PIC simulation 

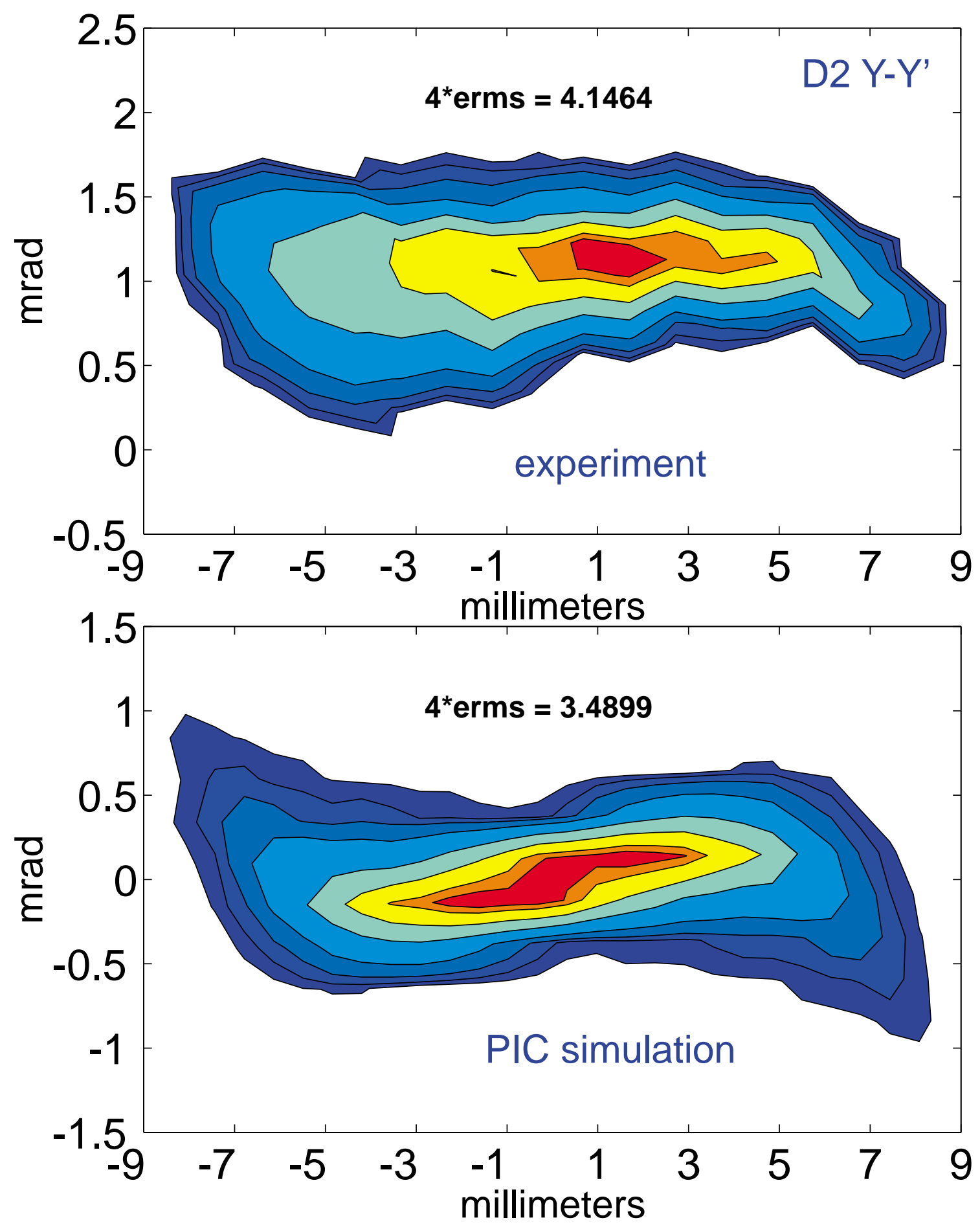

Figure 6.3: Transverse phase space at D2; measured and from PIC simulation 
Phase space measurements at D4 are important to determine the size and convergence angle of the beam on its way to the focus. These parameters must be quite close to the desired envelope in order for the beam to reach a waist at the desired drift distance corresponding to the (scaled) radius of the fusion reactor chamber. The 3-axis diagnostic at D5 is situated to measure the beam waist anywhere within $\pm 8 \mathrm{~cm}$ of this predicted focal spot location. Figures 6.4 and 6.5 show the horizontal $\left(\mathrm{X}-\mathrm{X}^{\prime}\right)$ and vertical $\left(\mathrm{Y}-\mathrm{Y}^{\prime}\right)$ transverse phase space distributions with the WARP data for comparison. While some of the features exhibited by the distributions are similar, there are some noticeable differences. There appear to be low intensity "fins" in the simulation near the center of the beam in both planes. Because these features are at the $2-5 \%$ level, they would most likely have been washed out by the noise on the experimental signal.

While the external focusing fields in the WARP simulation are perfect quadrupoles, the magnetic fields in the experiment are of course made up of a superposition of fields from discrete conductors. This represents a significant physical difference in the quality of the fields near the edge of the magnets. In both of the plots of experimental data, the "S"-shaped distortion that appears at D2 seems to have transformed into a "Z"-shape. For the WARP run, the vertical plane shows signs of this "Z"-shape tendency, while the horizontal plane seems to have retained the "S" shape. Because this "S" versus " $Z$ " discrepancy involves the edges of the beam, it is possible that it is due to additional higher order multipoles from the discrete nature of the focusing field, which has the largest effect near the magnet bore radius. 

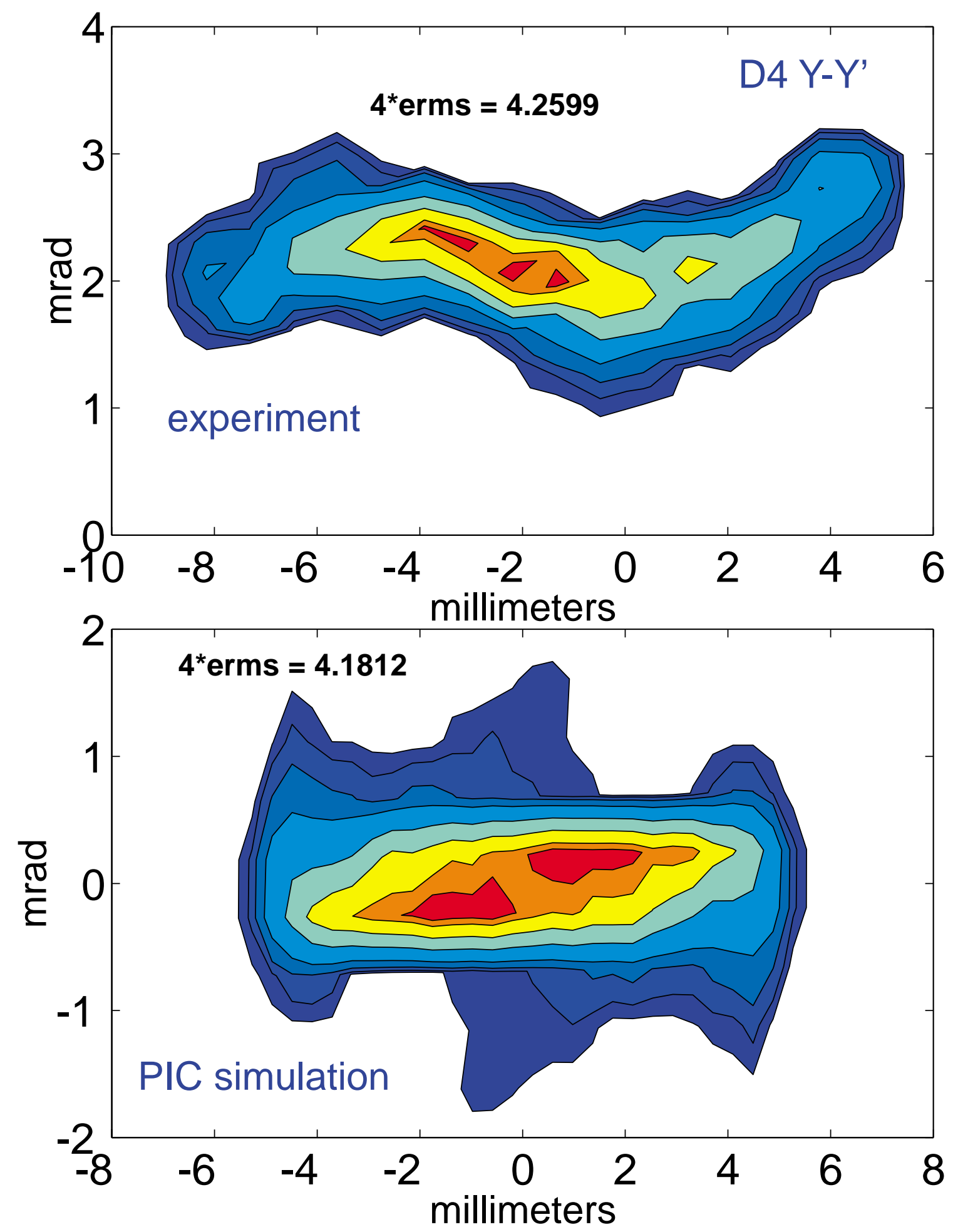

Figure 6.4: Vertical transverse phase space at D4; measured and from PIC simulation 

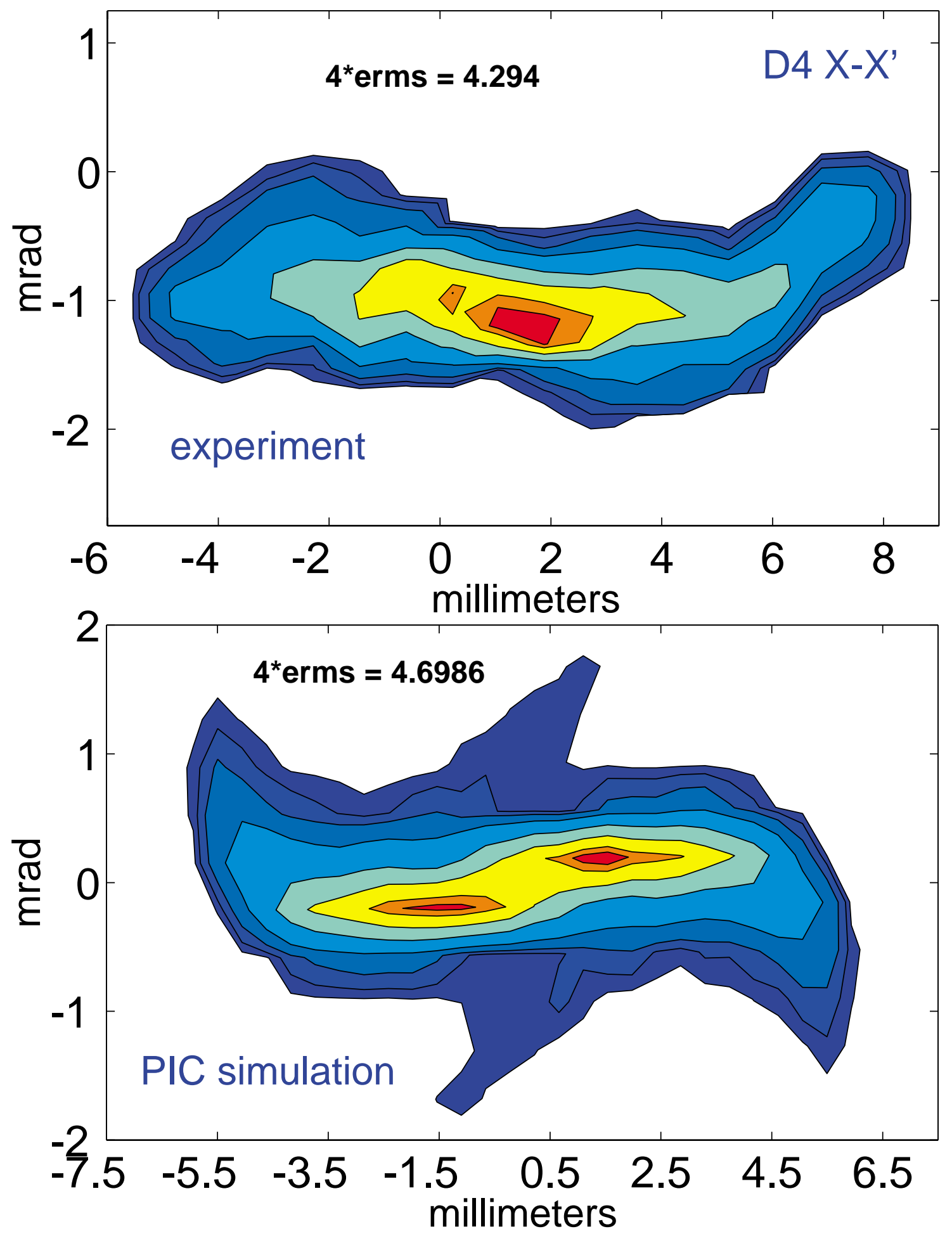

Figure 6.5: Horizontal transverse phase space at D4; measured and from PIC simulation 
The crossed slit measurement technique described in Section 3.4 is used to examine the evolution of the beam current density in the focusing system. Figure 6.6 compares measurements made at D1 and D4 using a $1 \mathrm{~mm}$ by $1 \mathrm{~mm}$ grid. Included is a plot of the amplitudes versus position for a slice of the beam through the middle of the D1 measurement. The Debye length of the experimental beam calculated using the measured emittance is $0.3 \mathrm{~mm}$, or about $6 \%$ of the beam radius. The measured current density profile closely resembles that of a Maxwell-Boltzmann distribution for a space charge dominated beam. Like the K-V distribution described in Section 2.1, the transverse Maxwell-Boltzmann or thermal distribution is a solution of the time independent Vlasov equation, and best describes the equilibrium state of a real beam (Reiser, p. 379-83). For a space-charge dominated beam, this distribution is characterized by $\lambda_{D}<<a$ and a "flat top" region extending out to approximately $0.6 a$. Later, at D4, the flat top of the current density distribution has become more rounded. Both the increase in the emittance from D1 to D4 and the non-linear components of the space-charge and focusing fields contribute to the evolution of the density profile. 

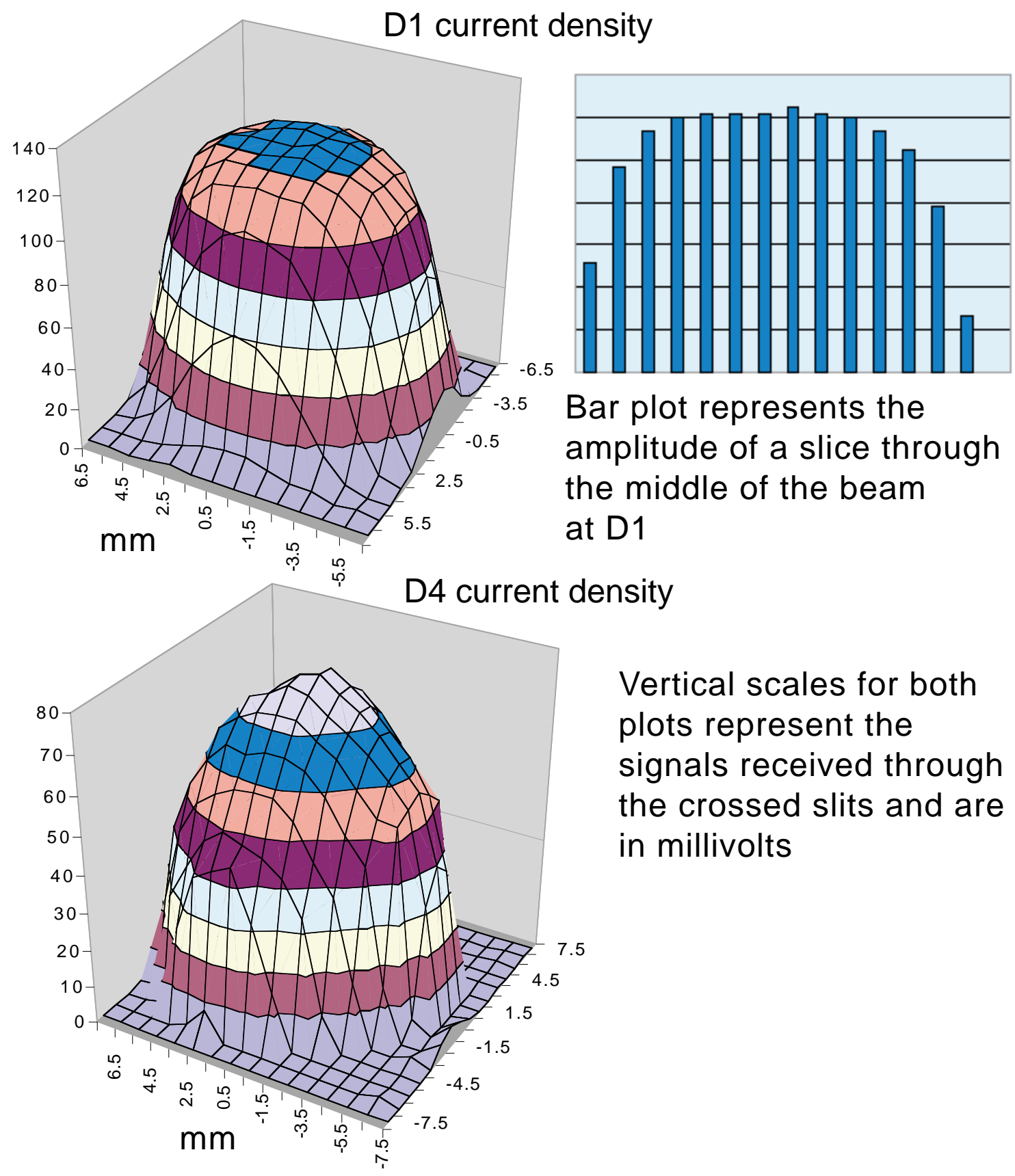

Vertical scales for both plots represent the signals received through the crossed slits and are in millivolts

Figure 6.6: Crossed slit measurements of beam current density at D1 and D4. For each measurement, the slit widths and the step size intervals are $1 \mathrm{~mm}$. 


\subsection{Predicted Spot Characteristics}

As mentioned in Chapter 2, the envelope equation can be used to predict the minimum radius of the beam for a given convergence angle from the last focusing element. First, we write Equation 2-13 for a round beam of radius $r$ in a region with no external focusing fields,

$r^{\prime \prime}=\frac{Q}{r}+\frac{\varepsilon_{x}^{2}}{r^{3}}$

Multiplying by $r^{\prime}$ and integrating along the axial direction,

$\frac{1}{2}\left[\left(r_{f}^{\prime}\right)^{2}-\left(r_{o}^{\prime}\right)^{2}\right]=Q \int_{r_{o}}^{r_{f}} \frac{d r}{r}+\varepsilon^{2} \int_{r_{o}}^{r_{f}} \frac{d r}{r^{3}}$

Here $r_{o}$ is the radius of the beam at the exit of the last magnet, and $r_{f}$ is the minimum radius or spot size. Their derivatives are equal to $\theta$, the convergence angle, and zero, respectively. Therefore,

$-\theta^{2}=2 Q \ln \left(\frac{r_{f}}{r_{o}}\right)-\frac{\varepsilon^{2}}{r_{f}^{2}}$

where we have used $r_{f}^{2}<<r_{o}^{2}$. Considering that the convergence angle and the initial radius are related by the focal length, $L$, we can re-write Equation 6-3 as $\theta^{2}-\frac{\varepsilon^{2}}{r_{f}^{2}}=2 Q \ln \left(\frac{\theta L}{r_{f}}\right)$

This equation is in general not solvable analytically for the spot size, $r_{f}$, but it can be solved graphically by plotting both sides as a function of the spot size. More intuitively, Equation 6-4 can be examined in the limit $\frac{\varepsilon^{2}}{r_{f}^{2}}>>$; i.e. an emittance dominated focus:

$r_{f} \cong \frac{\varepsilon}{\theta}$ 
This relation illustrates the importance of the emittance of the beam at the end of the accelerator to the spot size and therefore the target efficiency. For a convergence angle that minimizes the effects of geometric aberrations $(\leq 20 \mathrm{mrad})$, the emittance available from the accelerator is balanced against the desired focal spot parameters of the target.

The opposite limit, that of a space charge dominated focus, may result from a driver design that favors a fewer number of beams incident on the target and thus a higher current per beam. This situation leads to a spot size that is incompatible with the target gains necessary for economic power generation as discussed in Chapter 1. Designs such as these rely on partial neutralization of the space charge of the converging beam to return the focus to the emittance dominated regime. Therefore, Equation 6-5 applies universally and often serves as a convenient tool for optimizing driver designs.

The nature of the current density distribution at the spot can be determined by comparing the two terms on the right side of Equation 6-1 (Reiser, 1994). The perveance term represents the self forces of the beam and is typically dominant during transport of the beam prior to the focus, when it is of the order of the external focusing force term, giving the beam the fairly uniform transverse density distribution shown in Figure 6.6. If this term is the dominant term at the focus, the beam should also exhibit this uniform density distribution and the profile generated by the single slit scan diagnostic should take on the cosine shape described in Section 3.4. On the other hand, if it is the emittance term that is dominant at the focus due to the $1 / \mathrm{r}^{3}$ dependence, the transverse temperature of the beam determines the shape of the distribution. The single slit scan will yield a Gaussian profile as a result of the Gaussian nature of the radial density at the spot. 
The ratio of the emittance term over the perveance term can be calculated for the various experimental conditions. Note that due to the factor of the radius that remains in the ratio, the result will be identical for the scaled experiment and the HIBALL II design. For the $95 \mu \mathrm{A}$ (HIBALL II) beam, the parameters at the spot yield a ratio of 5.8, indicating that the profiles measured should have a Gaussian shape. In the next chapter, results from the $400 \mu \mathrm{A}$ beam will be presented. These measurements yield a ratio of 0.2 , placing the focus in the space charge dominated regime. Neutralizing this beam with electrons, however, will have a significant effect on the ratio. A neutralization fraction of $80 \%$ will reduce both the perveance and the spot size, resulting in a ratio of 7.3 . Neutralizing the focus should therefore have a pronounced effect on the spot profile.

We can first perform the calculation outlined above for the HIBALL II design. The report uses a convergence angle of 9 mrad and a focal length of 8.5 meters for the 10 $\mathrm{GeV} \mathrm{Bi}^{+}$beam. Using this data along with a current of 1250 amperes and an emittance of $30 \pi \mathrm{mm}$ mrad, we plot the two sides of Equation 6-4 as functions of radius in Figure 6.7. The right hand side is plotted with the red curve and the left hand side with the blue curve, obtaining a value of $5.8 \mathrm{~mm}$ for the calculated spot size. Figure 6.8 repeats the calculation for the scaled experiment. Here, the convergence angle is also $9 \mathrm{mrad}$, but the measured value of the emittance is used to produce a beam radius of $0.65 \mathrm{~mm}$, slightly larger than the one-tenth scaled design spot radius. 


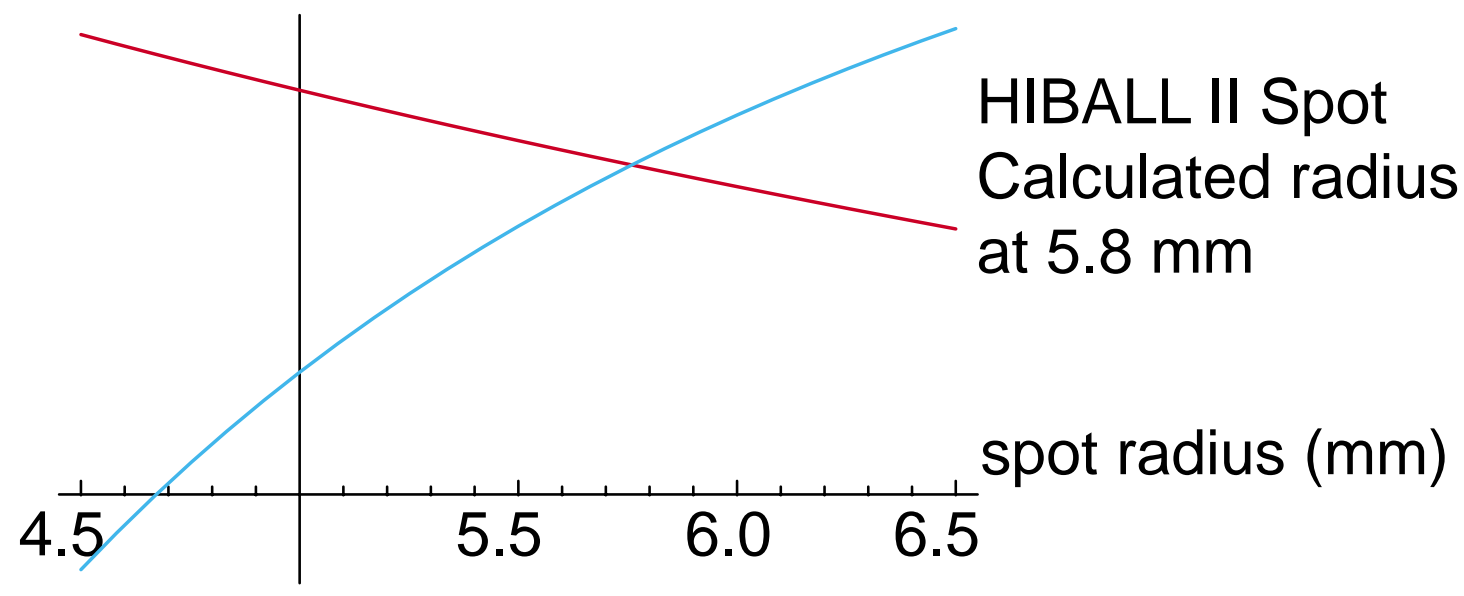

Figure 6.7: Graphical solution of Equation 6-4 for the HIBALL-II design parameters

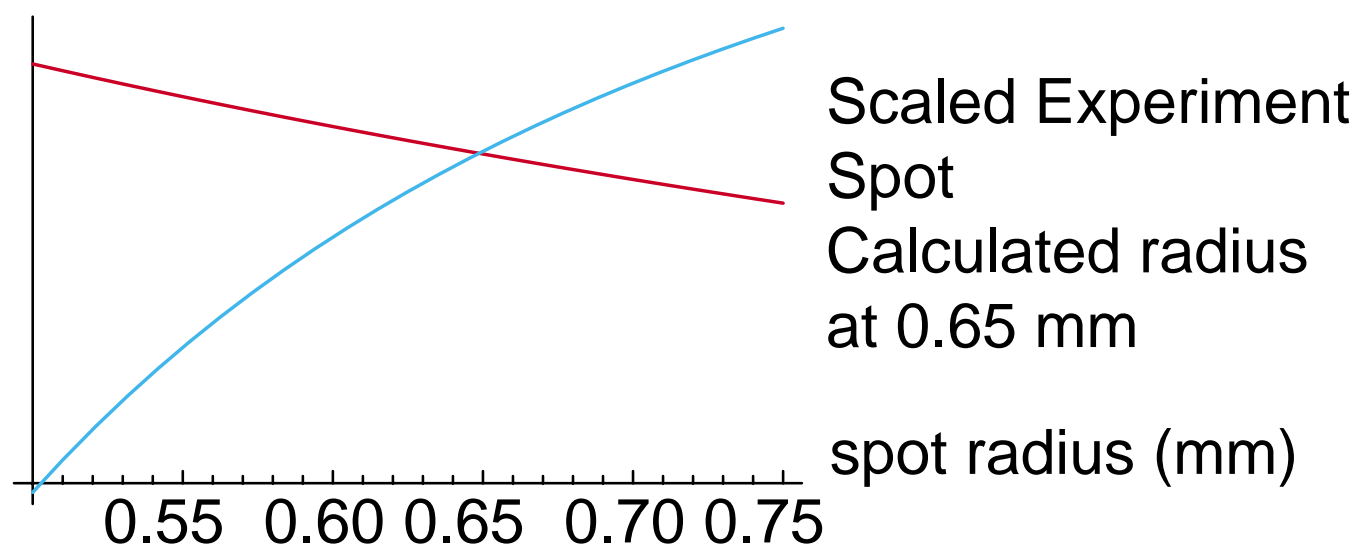

Figure 6.8: Graphical solution of Equation 6-4 for the measured experimental parameters 


\subsection{Spot Measurements}

Once the magnetic focusing solution has been tuned to match the beam sizes and focusing angles in both planes at D4, the focal spot can be measured using the 3-axis probe described in Section 3.4. Single slit measurements are made at a series of axial locations to identify the beam waists in the $\mathrm{x}$ - and $\mathrm{y}$ - planes. Additional fine-tuning of the last magnetic quadrupole causes these waists to coincide at the same axial location. Then measurements at the waist are made using a $50 \mu \mathrm{m}$ slit and $100 \mu \mathrm{m}$ steps for high resolution. In light of the argument presented in the previous section, the measurements are fit with Gaussian functions to extract a spot radius.

Figure 6.9 shows the results of horizontal and vertical scans taken with the 3-axis probe at the same axial location. The portion of the beam that passes through the slit creates a signal that is proportional to the current density, and the raw data is plotted as millivolts. The results of the Gaussian fit show that the beam is almost round with a twice rms radius of $0.5 \mathrm{~mm}$. This is smaller that the $0.65 \mathrm{~mm}$ calculated in the previous section using the emittance measured at the D4 location. Alternately, using the twotimes-rms radius from the second moment of the raw data, the horizontal radius is 0.75 $\mathrm{mm}$ and the vertical radius is $0.57 \mathrm{~mm}$. These results bracket the calculated value. Further analysis of the data is helpful in order to evaluate the performance of the experimental beam with respect to a round target.

Figure 6.10 plots the data from the same measurements on different axes. Here the signal is integrated, normalized, and plotted as a function of distance from the centroid of the distribution. This yields the fraction of the ions that strike within a given distance from the center, projected onto either the horizontal or vertical axis. On this plot 
the results of the WARP simulation at the focal spot are also presented. To achieve a valid comparison with the measurement, the macro-particles are collapsed onto a transverse axis and binned at $100 \mu \mathrm{m}$ intervals. The bins are integrated in the same manner as the data and plotted as the green curve. To compare these results with the HIBALL-II design, a Gaussian profile is assumed for the focal spot, and the twice rms radius calculated earlier $(0.58 \mathrm{~mm})$ is assigned as $2 \sigma$. The profile is projected onto the transverse axis, and the integrated current versus position is represented by the blue curve.

Because of the projective nature of the diagnostics, it is not possible to plot a unambiguous representation of the fraction of the experimental current that falls within a given radius. However, one can assume that the current density at the spot has a Gaussian form with independent widths in the $x$ and $y$ transverse dimensions. Then, a fraction-versus-radius curve can be generated by numerically integrating the function at a series of radii and plotting the result. This is done in Figure 6.11 for both the widths from the Gaussian fits (red curve) and from the second moments of the raw data (green curve). The same calculation was performed using the calculated HIBALL-II width and an axially symmetric Gaussian function $\left(\sigma_{x}=\sigma_{y}\right)$ and added to the plot (blue curve). For most of the range of the vertical axis of the figure, the red and green curves serve as boundaries for the actual fraction of the experimental beam. 

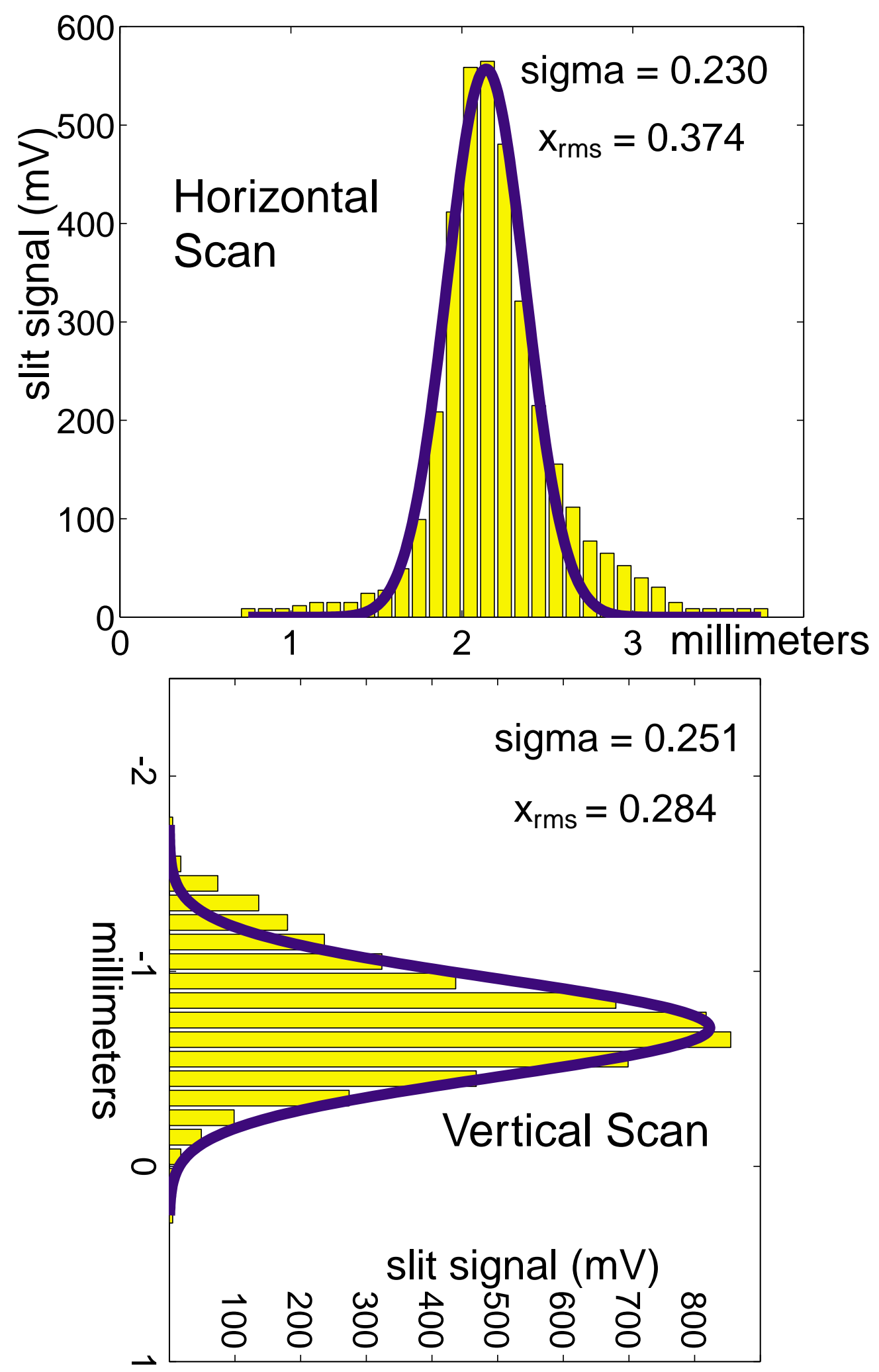

Figure 6.9: Single slit scans in both transverse planes at the focal point. Widths of the raw data $\left(\mathbf{x}_{\mathrm{rms}}\right)$ and of the Gaussian fit $(\sigma)$ are indicated. 

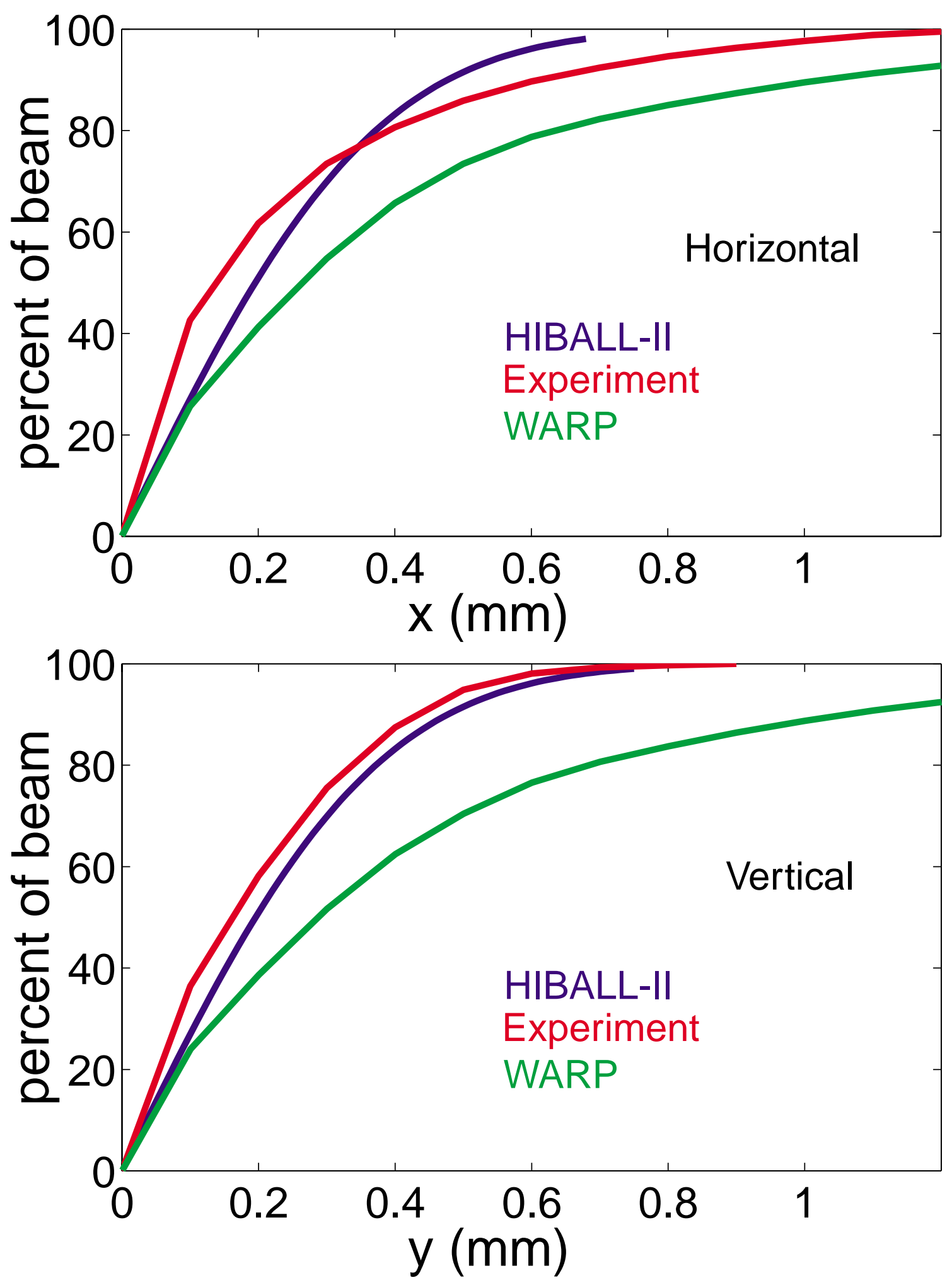

Figure 6.10: Percent of the beam current at the focus as a function of distance from the centroid of the distribution. 


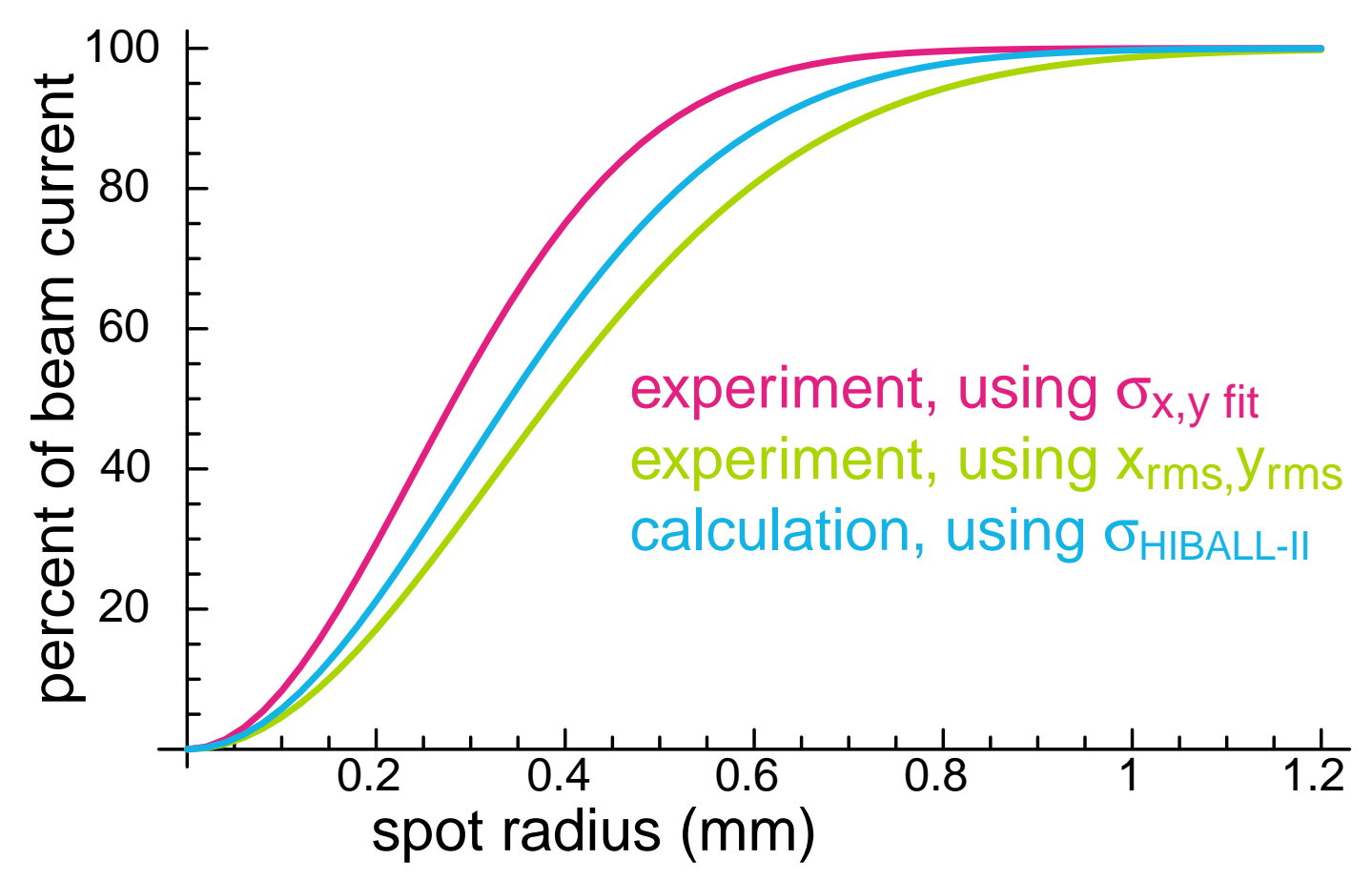

Figure 6.11: Percent of beam current at the focus as a function of radius assuming a two dimensional Gaussian distribution.

\subsection{Chromatic Aberration Studies}

Aberrations can have a considerable effect on a focal spot whose size would otherwise be determined purely by the perveance and emittance of the beam. As mentioned in Section 1.4, a chromatic aberration is caused by a deviation from the nominal energy expected for the focusing solution. Because the external focusing force for the magnetic quadrupole lattice is inversely proportional to the momentum, the trajectories of the particles are changed throughout the system, causing a shift in the axial location of the beam focus in each plane. The result is a focal spot that is no longer round, having a greater area than the nominal energy case. In a real driver, there will be a spread about the nominal energy due to the broadening of the longitudinal momentum distribution that results from compression of the beam pulse. Additionally, there will 
most likely be a residual end to end "tilt" of the momentum from this compression as the beam passes through the final focus sub-system.

To study the chromatic aberration experimentally, it would be desirable to adjust the beam energy at the few percent level holding other parameters fixed. The experimental beam would then represent a single "slice" of the driver beam with each particle having the same maximum value of energy deviation. However, the Child Law governing extraction of ions from the source states that the beam current will increase as $V_{\text {diode }}^{\frac{3}{2}}$ (Humphries, 1990, pp. 195-200). Also, one would want to vary the energy only in the magnetic section to prevent the effects of an energy shift in the electrostatic section from confusing the results. To avoid these issues, an energy shift is studied with the experiment by de-tuning the focusing gradients in a uniform manner. That is, the strengths of the pole tip fields in each of the six magnets are changed by the same fraction to simulate the deviation in beam energy.

Actually, each of the terms in the envelope equation would be affected by a shift in energy. For the Final Focus experiment, however, both the emittance and the perveance terms are small compared to the focusing force term when the beam is in the magnets. This assumption is checked by modeling the chromatic effect in the envelope code both with a real energy shift and also a uniform adjustment to the magnet strength. In the experiment, measurements are made using the $95 \mu \mathrm{A}$ beam with the magnetic quadrupole strengths de-tuned by $\pm 0.5 \%$, and $\pm 1.0 \%$, corresponding to energy shifts of $\pm 1 \%$, and $\pm 2 \%$. Because the target position in a driver would be fixed in space, the beam horizontal and vertical profiles are measured at the same axial location as in the nominal energy case. 
Figure 6.12 shows the fractional increase in the area of the focal spot for each of the cases. The red bars are the experimental measurements comparing the product of the widths of both planes to the product for the non-shifted case. The blue bars represent a model of the experiment using a code that integrates the envelope equation through the experimental focusing lattice. The code is very useful in this case as it permits the focusing fields to be either electrostatic or magnetic. The input allows for manipulations of the strength of these fields as well as the beam energy. The dark blue bars represent the spot that results when each of the magnet strengths are shifted by the appropriate percent. The light blue bars are for the corresponding energy deviations; here the focusing strengths are left alone, and the beam energy is shifted. The agreement between the two sets of blue bars implies that the effect of the energy shift on the terms other than the external focusing force is a small one for the given system, as presumed in the previous paragraph.

Figure 6.13 illustrates the affect of the shifts on the two measurement planes in detail. The data from the slit scans is presented in the same format as in Figure 6.10, plotting the fraction of ions that strike within a given distance from the centroid. From Figures 6.12 and 6.13 , we see that the $\pm 1 \%$ energy shifts increase the spot size by approximately $20 \%$, and the $\pm 2 \%$ by $50-70 \%$. The data is in fairly good agreement with the calculations of the code, except for the $+2 \%$ case. While the code assumes four-fold symmetry at the focus, it is clear from Figure 6.9 that the beam does not display this type of symmetry; this detail may have a role in the discrepancy. 


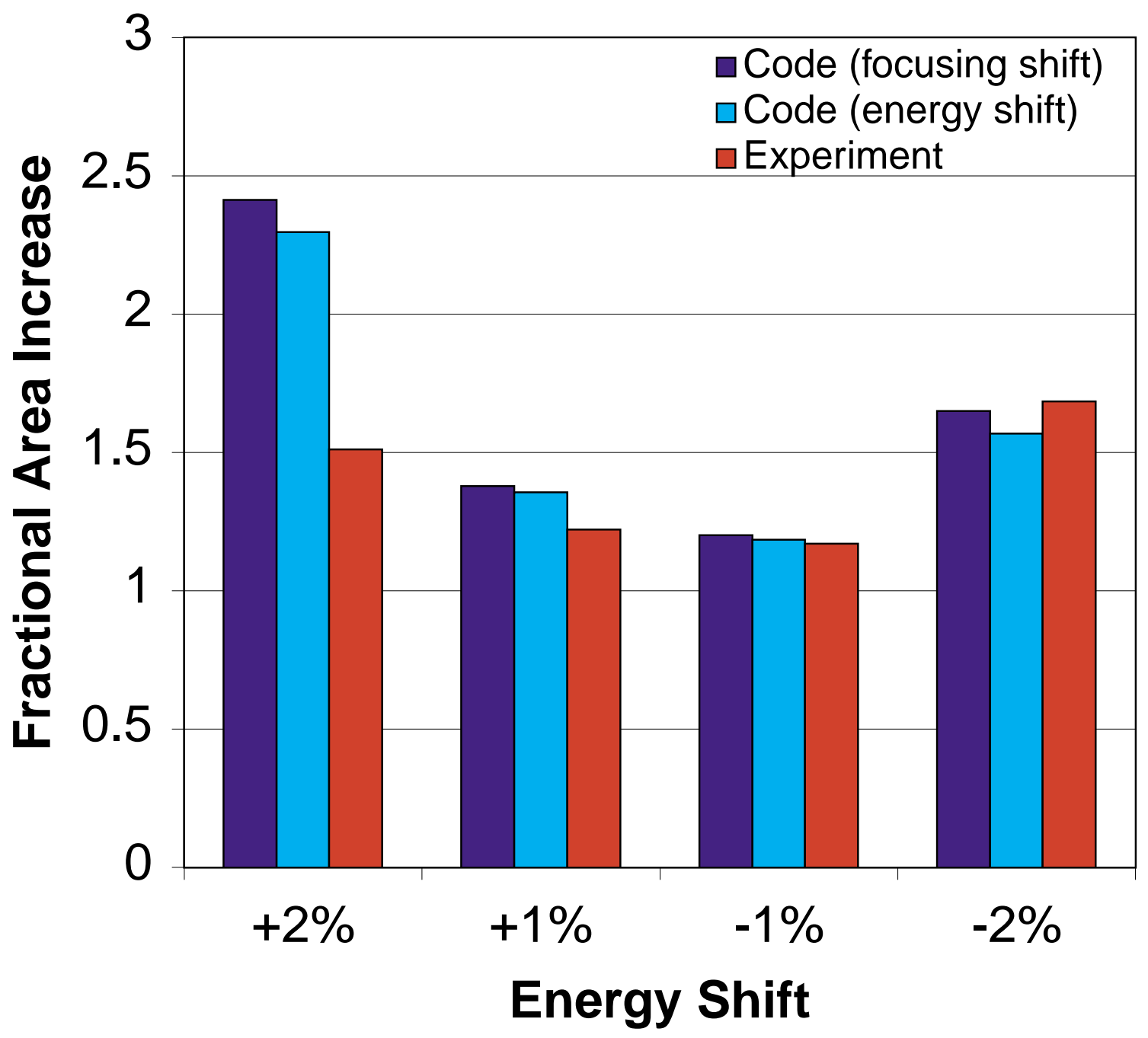

Figure 6.12 :Fractional increase in focal spot area for each of the energy shift trials.

The results of a similar study are discussed in the HIBALL-II report. Here, the ion optics code used for the design of the final focus system tracks a monochromatic beam through the lattice at a series of energies. The results are plotted as a fraction of the current that strikes within the desired radius $(4 \mathrm{~mm})$ compared to the on-momentum case (Wollnik, 1985). To plot the experimental results on the same axes, a Gaussian distribution is assumed for the data as was done for Figure 6-11. For each of the momentum variations, a distribution was constructed based on the second moments of the focal spot data in each plane and integrated out to the equivalent scaled radius $(0.4 \mathrm{~mm})$. 
To obtain an "efficiency" value, the result was normalized to the integral of the onmomentum data to the same radius. These calculations, along with the data from the HIBALL-II report are presented in Figure 6-14. 


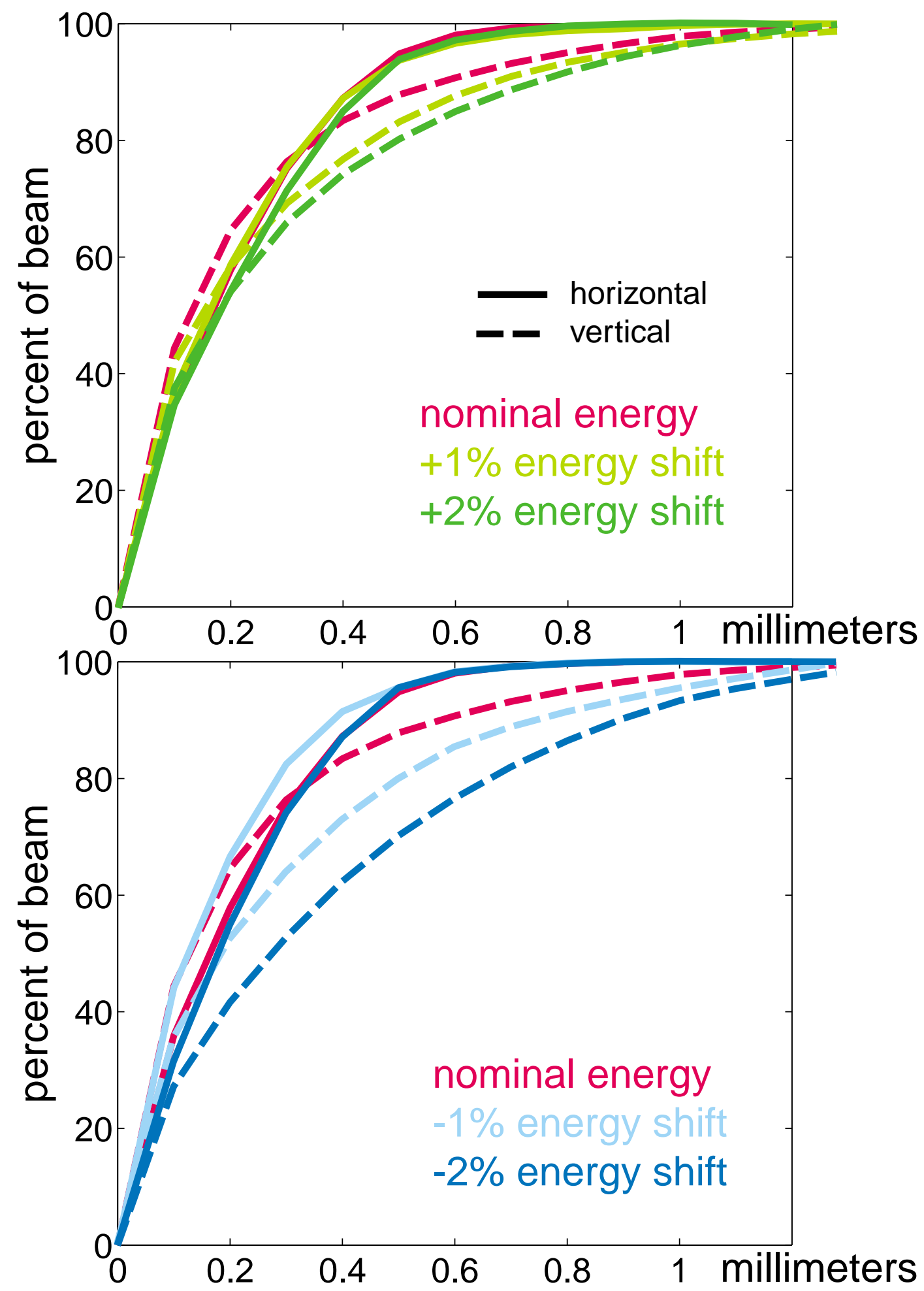

Figure 6.13: Percent of the beam current at the focus as a function of distance from the centroid of the distribution for each of the energy shift trials. Data from both the horizontal and vertical slit scans are shown on each plot. 


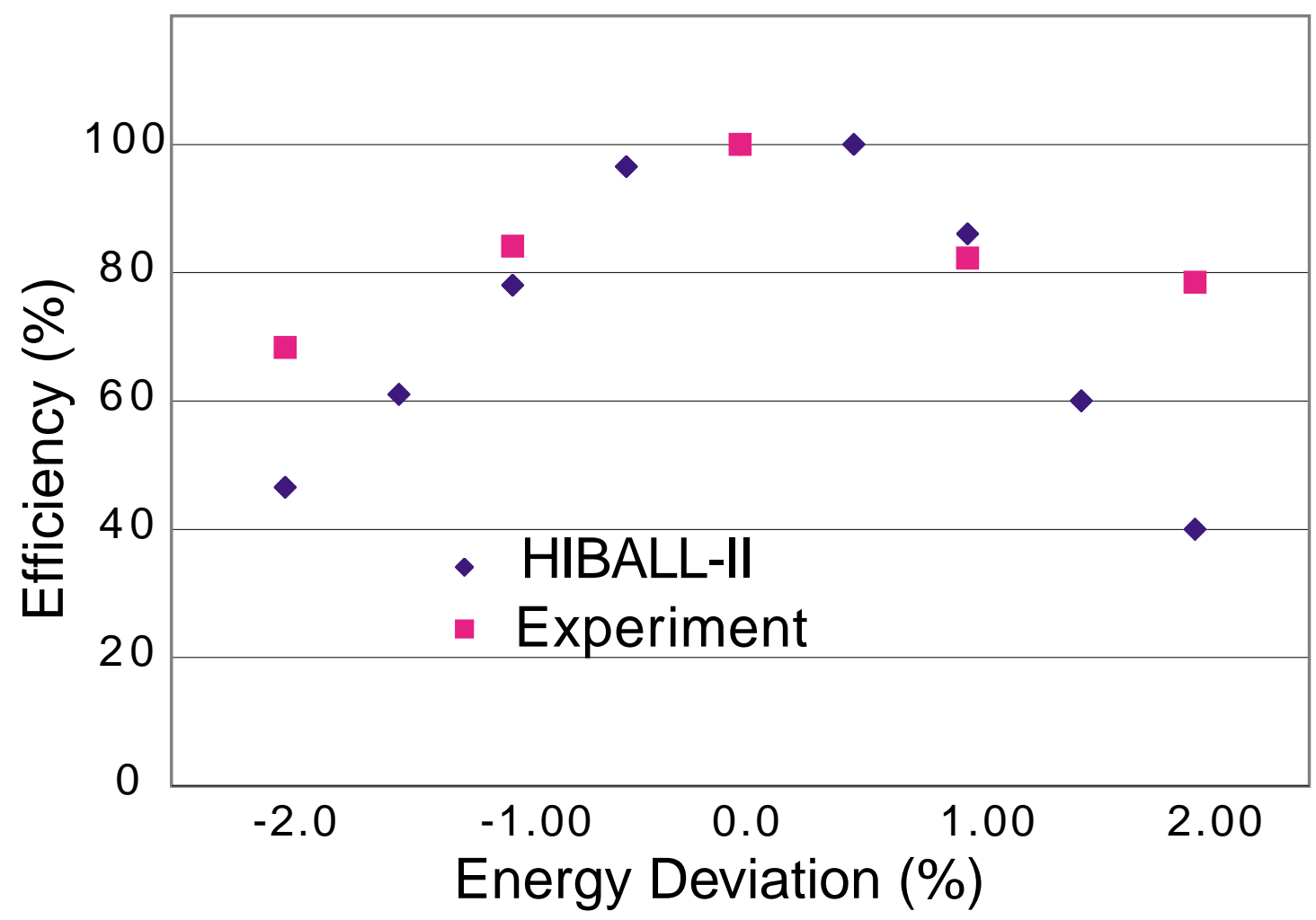

Figure 6.14: The fraction of the current within a $0.4 \mathrm{~mm}$ radius is normalized to the onmomentum measurement and plotted as "Efficiency" for comparison to the HIBALL-II report. 


\section{Neutralized Focus Results}

\subsection{The Higher Current Solution}

As mentioned in the introduction, various issues associated with the fusion chamber in an IFE power plant may cause neutralization of the beam to be unavoidable. To measure the effect of electron neutralization on the experimental beam focal spot, it is desirable to employ a beam with a large enough current that without neutralization would remain space charge dominated through the focus. Then a change not just in the size but also in the shape of the focal spot may be observed. Using the envelope code model of the experiment described in Section 5.3, the maximum beam current allowable given the dimensions of the experiment was determined to be approximately $400 \mu \mathrm{A}$. This factor of four increase in the perveance is equivalent to $5 \mathrm{kA}$ of $10 \mathrm{GeV} \mathrm{Bi}^{+}$in the HIBALL-II design, and produces a space charge dominated focus for the same convergence angle as the lower perveance case.

If the convergence angle to the spot and the emittance are held constant, the beam radius can be plotted as a function of the current by solving Equation 6-4 for various values of the perveance. This result is presented in Figure 7.1 as the blue curve, with the low and high perveance conditions indicated as points on the curve. The red curve represents the boundary between the space charge- and emittance-dominated regimes. This curve is found by setting the perveance and emittance terms from Equation 6-1 equal, and solving for the beam current using Equation 2-12. The result is plotted as a function of radius. Together, the two curves show that a $400 \mu \mathrm{A}$ beam, neutralized by approximately $75 \%$, can be observed to have an emittance dominated focus with a radius 
of approximately $1.25 \mathrm{~mm}$. Thus, both the size and shape of the focal spot should change measurably.

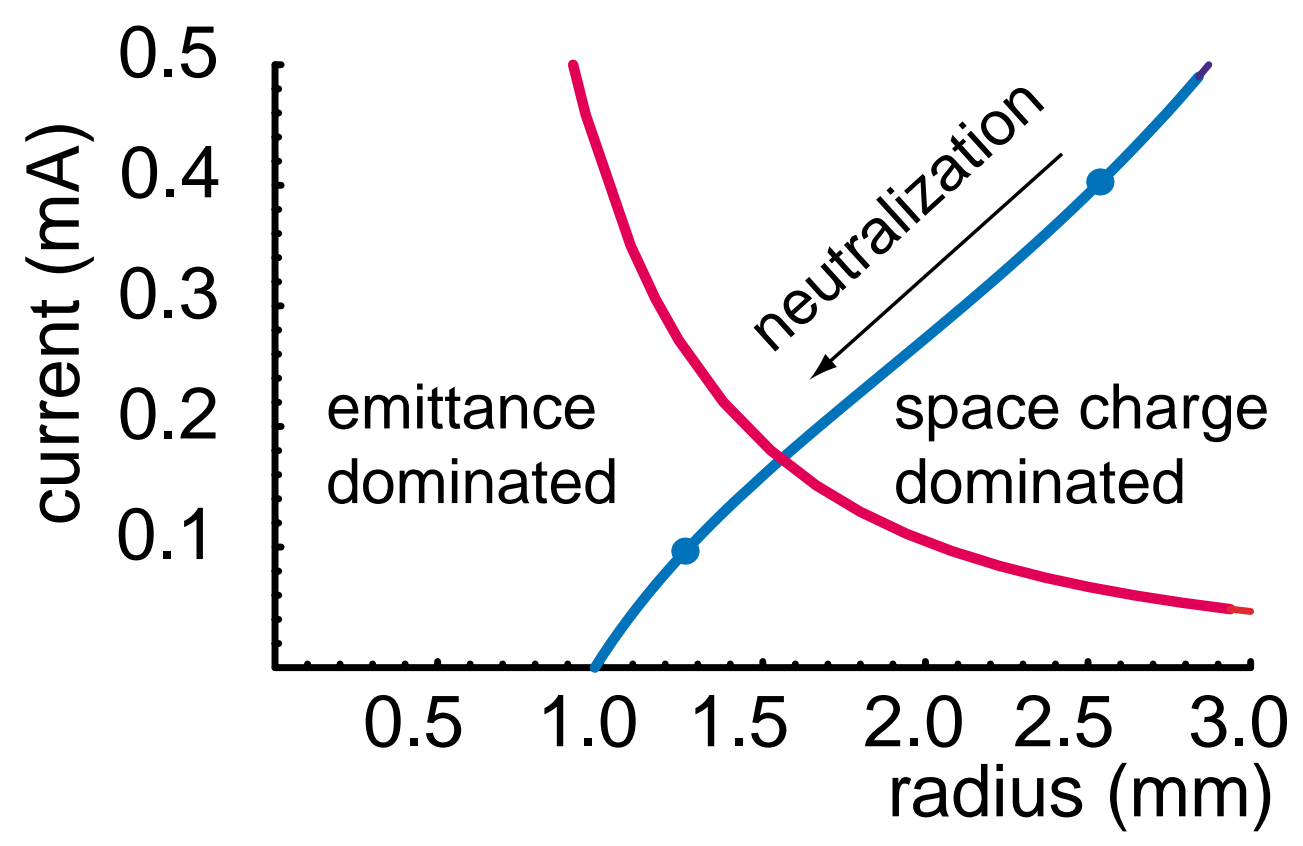

Figure 7.1: Spot radius is plotted as a function of beam current leaving other parameters fixed, with the two points indicating the experimental values of the current. The red curve separates the emittance and space charge dominated regimes.

A $2 \mathrm{~mm}$ aperture to extract the $400 \mu \mathrm{A}$ current was installed in the wheel at the exit of the diode. The beam envelope used for this current is illustrated in Figure 7.2 in a similar manner as Figure 6.1. Comparing the two figures, it is immediately apparent that the $400 \mu \mathrm{A}$ beam fills a considerably larger fraction of the aperture in most of the focusing elements. For the higher current beam, it is necessary to use the first ten electrostatic quadrupoles instead of only four in order to control the beam expansion from the aperture. In order to better fit the envelope through the magnetic quadrupoles, the beam is elliptical during the drift from the electrostatic section. The end result is that the beam envelope parameters, radii and convergence angles, are the same at D4 as for the 95 $\mu$ A case. 


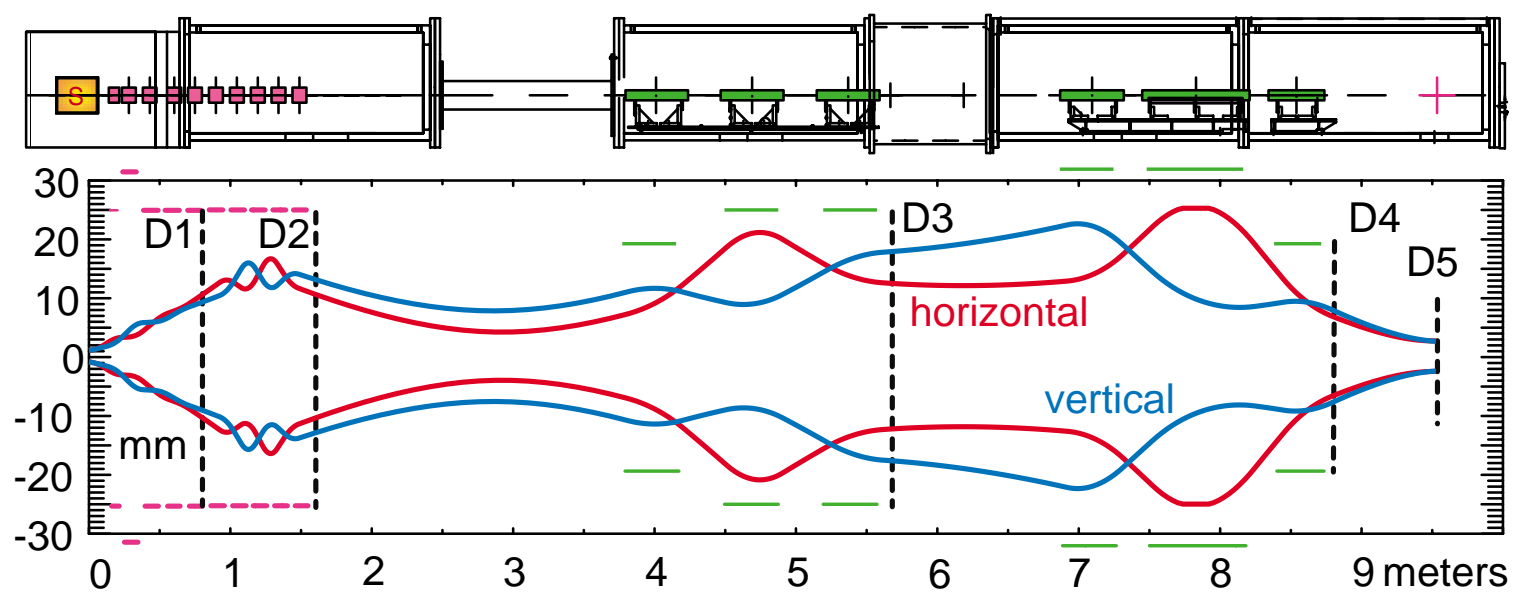

Figure 7.2 Horizontal and vertical envelopes calculated for the $400 \mu \mathrm{A}$ beam

As was done in the previous chapter, the phase space plots at each diagnostic station are compared with results from the 2D WARP simulation. Figure 7.3 shows the results at the first diagnostic station, and Figure 7.4 plots the phase space at D2, at the end of the electrostatic quadrupoles. An immediate result of the expansion to $\sim 15 \mathrm{~mm}$ in the electrostatic section is the enhancement of the " $\mathrm{S}$ " type phase space distortions by the time the beam reaches D2, and the shape and magnitude of the "S" are virtually identical in the simulation and the experiment. At this diagnostic, the degree of distortion and the emittance in the model more closely resembles the experiment. Again, these distortions arise in the WARP code as a result of the non-linear space charge forces in the expanding beam.

Figure 7.5 displays the horizontal phase space at D3. Note that in both the experiment and the simulation, the nature of the distortion has changed from an " $\mathrm{S}$ " type to a " $Z$ " type. Finally, in Figure 7.6, the vertical phase space at D4 is shown. The experimental beam seems to have become more peaked by this point, with the highest density contours confined to a smaller region along the $y$ axis. Here it is not clear if the nature of the distortions agrees between the two plots. At D3, the slit width artificially 

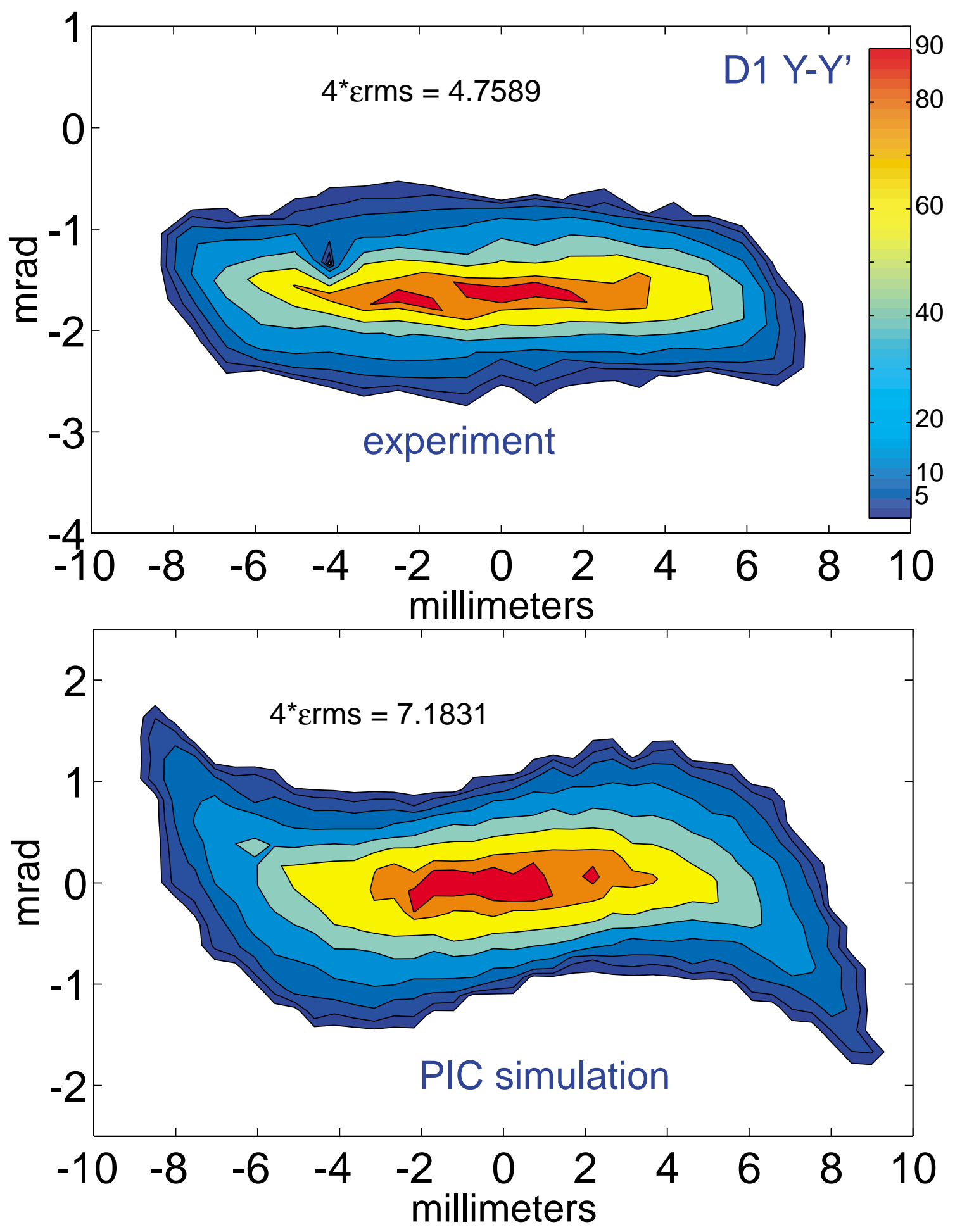

Figure 7.3: Transverse phase space at D1; measured and from PIC simulation 

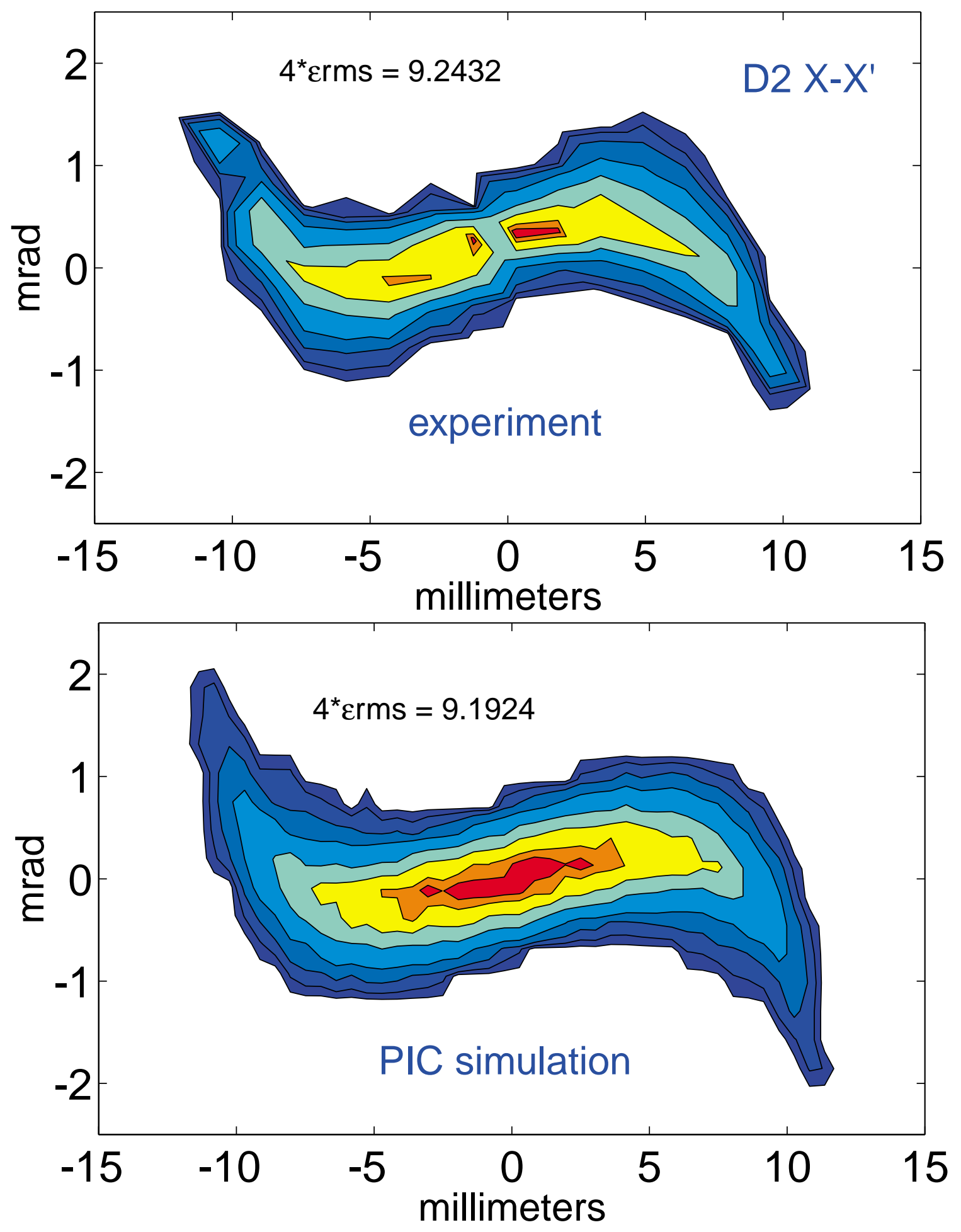

Figure 7.4 : Transverse phase space at D2; measured and from PIC simulation 

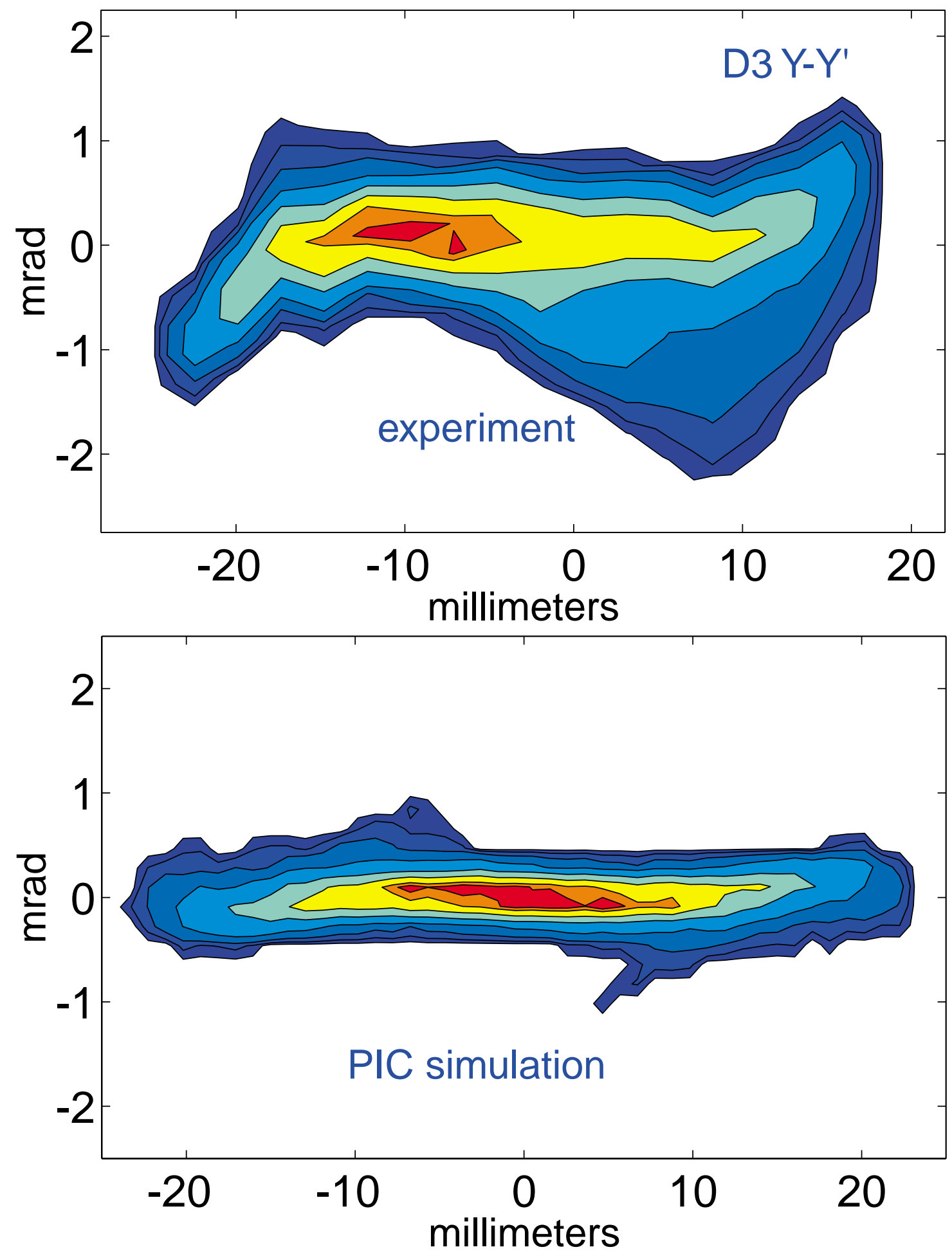

Figure 7.5 : Transverse phase space at D3; measured and from PIC simulation 

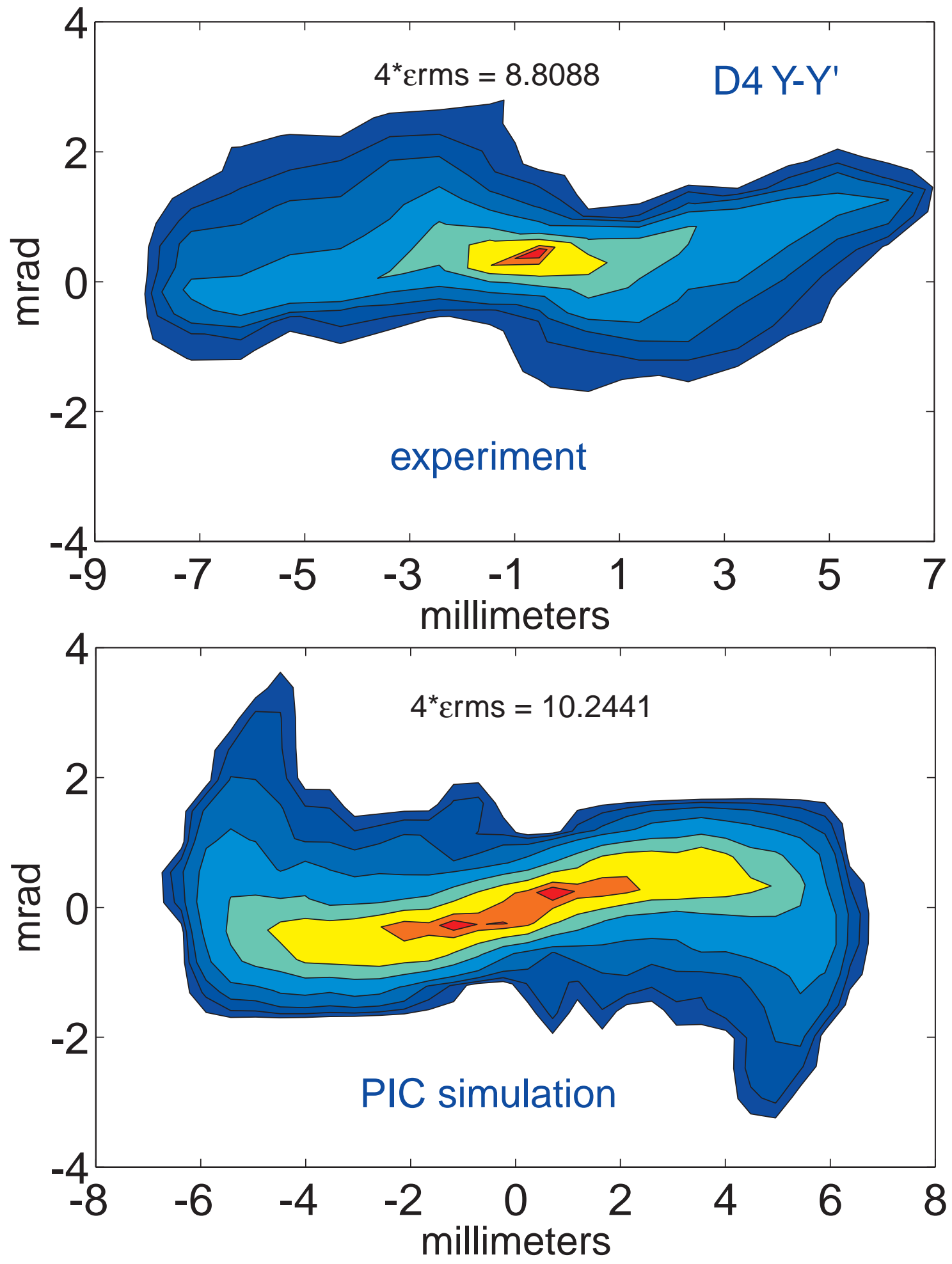

Figure 7.6 Transverse phase space at D4; measured and from PIC simulation 
enhances the transverse velocity distribution, preventing a proper measurement of the emittance as discussed in Section 8.2. The emittances can be compared again at D4, where the value for the simulation has grown since $\mathrm{D} 2$, while the experimental emittance has remained approximately constant. This is in agreement with the relative amplitudes of the distortion present in the two plots.

\subsection{Measurement of Neutralization with the Drift Tube}

Neutralization of the $400 \mu \mathrm{A}$ beam is accomplished with electrons emitted from the $75 \mu \mathrm{m}$ diameter hot tungsten filament located after the last magnetic quadrupole, described in Section 3.3. The fraction of the beam intercepted by the wire is measured to be $<1 \%$. Electrons born at the filament in the presence of the beam will be trapped within the space charge potential $\phi$ of the beam only if $e \phi>\frac{1}{2} m_{e} v_{i}^{2}$, where $v_{i}$ is the ion velocity (Olson, 1996). Treating the beam in the drift to the focus as a uniform cylinder of charge with boundaries at a distance much larger than the beam radius, $\phi \approx \frac{30 I}{\beta}$. For the experiment, the beam space charge potential is approximately $7.5 \mathrm{~V}$, while $\phi_{\min }$ is 0.7 $\mathrm{V}$. The maximum expected fractional neutralization is therefore $\sim 90 \%$.

Two types of measurements are made in an attempt to determine the fractional neutralization of the beam. One method is to simply measure the resulting spot size and infer the neutralization fraction; these measurements are discussed in the next section. The other measurement uses the drift tube diagnostic described in Section 3.4 and located in the drift region between the last magnet and the focus. This drift tube can be used to measure the difference between the net charge of the un-neutralized and neutralized beam. 
As the beam passes through the drift tube, a current flows away from the tube to create a net image charge on the tube surface. This signal is measured and integrated with the oscilloscope to produce a waveform that is proportional to the beam charge in the tube as a function of time. This waveform has the same characteristic shape as the beam current waveform that is recorded as the ions strike the collector of the Faraday cup. Therefore, the current measurement made at the Faraday cup co-located with the drift tube is used as a reference for the integrated tube waveforms. The tube signal is acquired and scaled to match the Faraday cup signal as is shown in Figure 3.11.

Typically, a small bias voltage is placed on the drift tube during the measurements. This bias is necessary to get the integrated tube signal to return to zero after the beam has left the tube. For the un-neutralized case, stray beam ions tend to strike the tube, and the secondary electrons that are produced leave a net positive charge on the tube. A very small positive bias voltage $(\approx 3 \mathrm{~V})$ is used to prevent the secondary electrons from leaving. In the case of the neutralized beam, electrons orbiting the beam that were emitted from the filament strike the tube leaving a net negative charge. A negative bias $(\approx 11 \mathrm{~V})$ is applied to the tube to prevent these electrons orbiting at large radius from entering the tube. In both cases the bias is adjusted until the integrated tube signal returns to zero once the beam has left the tube.

During initial measurements, it was discovered that as the head of the beam struck the 3-axis probe downstream, secondary electrons could travel back towards the drift tube and further interfere with the measurements. One of the 3 -axis probe slits is therefore centered on the beam spot, and the guard ring is energized. Thus, the same method of controlling the secondary electrons during the spot size measurements is employed for 
the drift tube measurements. When measurements have been made with the drift tube for both the neutralized and un-neutralized case, the scaling factors are compared to determine the degree of neutralization. The ratio of the scaling factors pointed to a neutralization fraction of $64 \%$.

\subsection{Measured Spot and Inferred Neutralization}

Measurements of the focal spot size are made at D5 in the same manner as described in Section 6.3. Each of the four measurements shown in Figure 7.7 (horizontal and vertical scans, neutralized and un-neutralized beams) is made at the same axial location. Both measurements are made using a $50 \mu \mathrm{m}$ slit with a $100 \mu \mathrm{m}$ step size for the neutralized focus and a $250 \mu \mathrm{m}$ step size for the case without neutralization. To emphasize the shift in the nature of the focus, the neutralized profiles are fit with Gaussian curves. The two values of $\sigma$ shown on each plot represent the value from the fit and the second moment of the raw data in the case of the neutralized and un-neutralized beam spots, respectively. The Gaussian nature of the neutralized density is clear; the vertical fit is extremely good, but the horizontal fit is affected by the asymmetry in the profile. From these plots the transition from space charge dominated to emittance dominated focus, shown graphically in Figure 7.1, is made clear.

As in Section 6.3, the data in Figure 7.7 represent time slices in the steady state region during the middle of the beam pulse. For purposes of illustration, Figure 7.8 plots the data from the slit scans in the vertical plane at D5 as a function of time. These are plots of the signal as it is intercepted by the collector at a single axial location and should not be confused with a snapshot in time of the entire length of the pulse. Both unneutralized and neutralized results are presented at the same scale. In both cases, the 


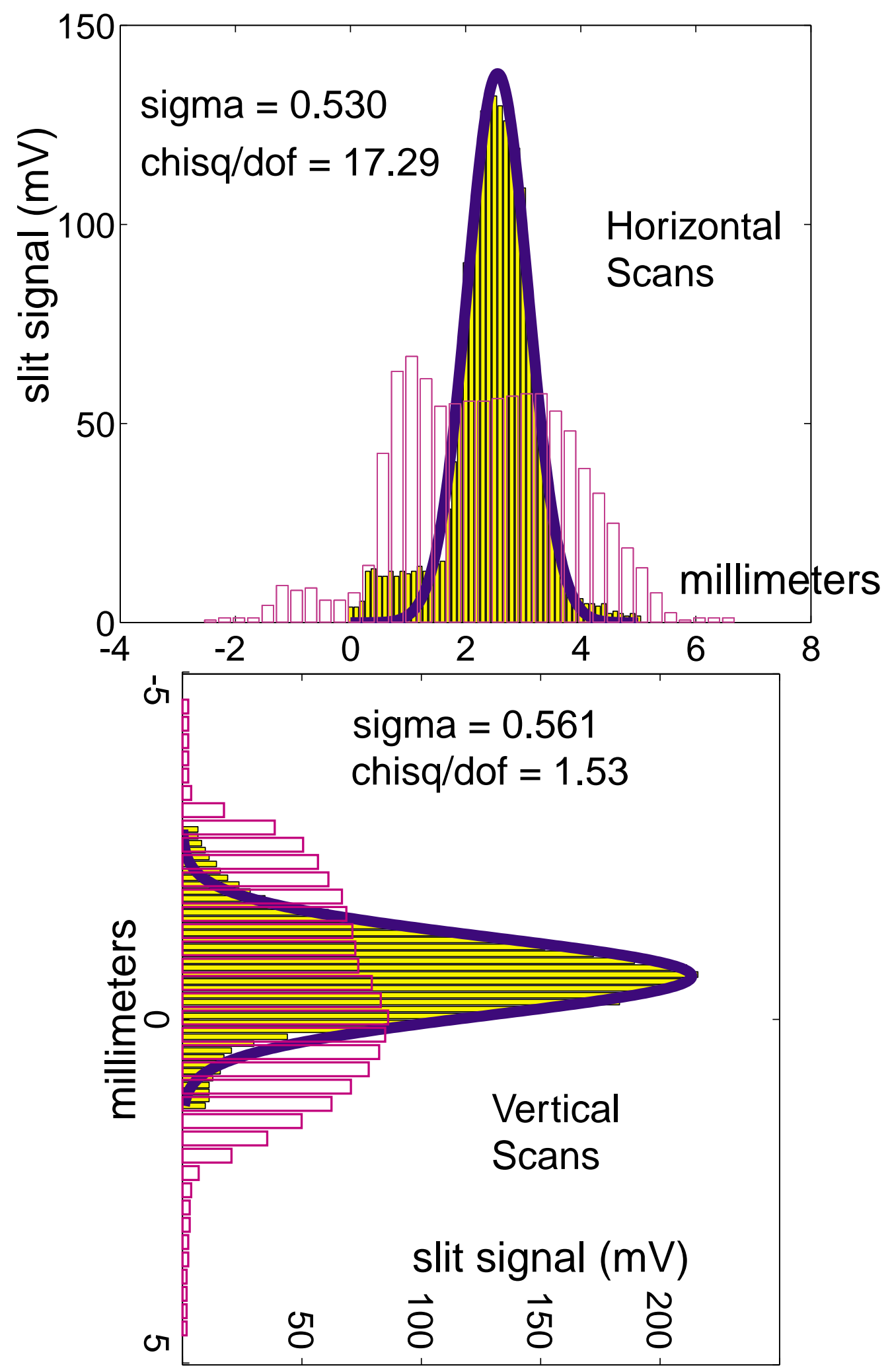

Figure 7.7: Single slit profiles of the $400 \mu \mathrm{A}$ focal spot. The hollow purple bars represent the un-neutralized focus, while the solid yellow bars represent the result of using the filament to neutralize the focus. 

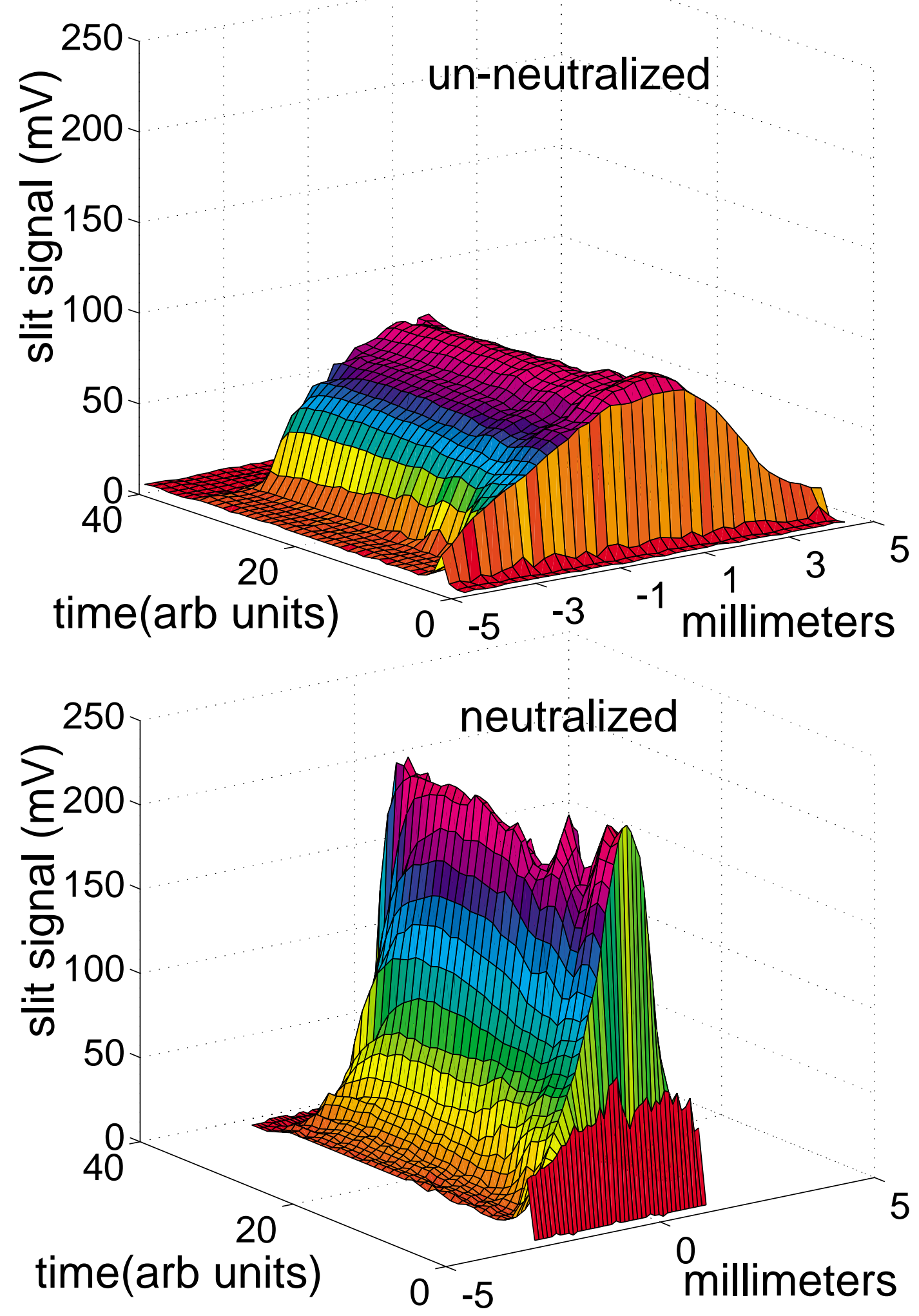

Figure 7.8: Time resolved slit measurement of the focal spot in the vertical plane 
current spike at the head of the pulse is apparent, followed by a region of relatively constant behavior before the tail occurs.

To determine the fractional neutralization from these measurements, a comparison is made between the data and the envelope equation (as employed in the envelope code described in Section 5.3). The beam radii, convergence angles, and emittance measured at D4 are input as initial conditions, and the code is run using the full current to produce the results measured without neutralization. The beam current is then reduced until the resulting spot agrees with the neutralized measurements. The two cases are illustrated in Figure 7.9. The fractional neutralization is then the ratio of the difference in current to the full current, $80 \%$ in this case.

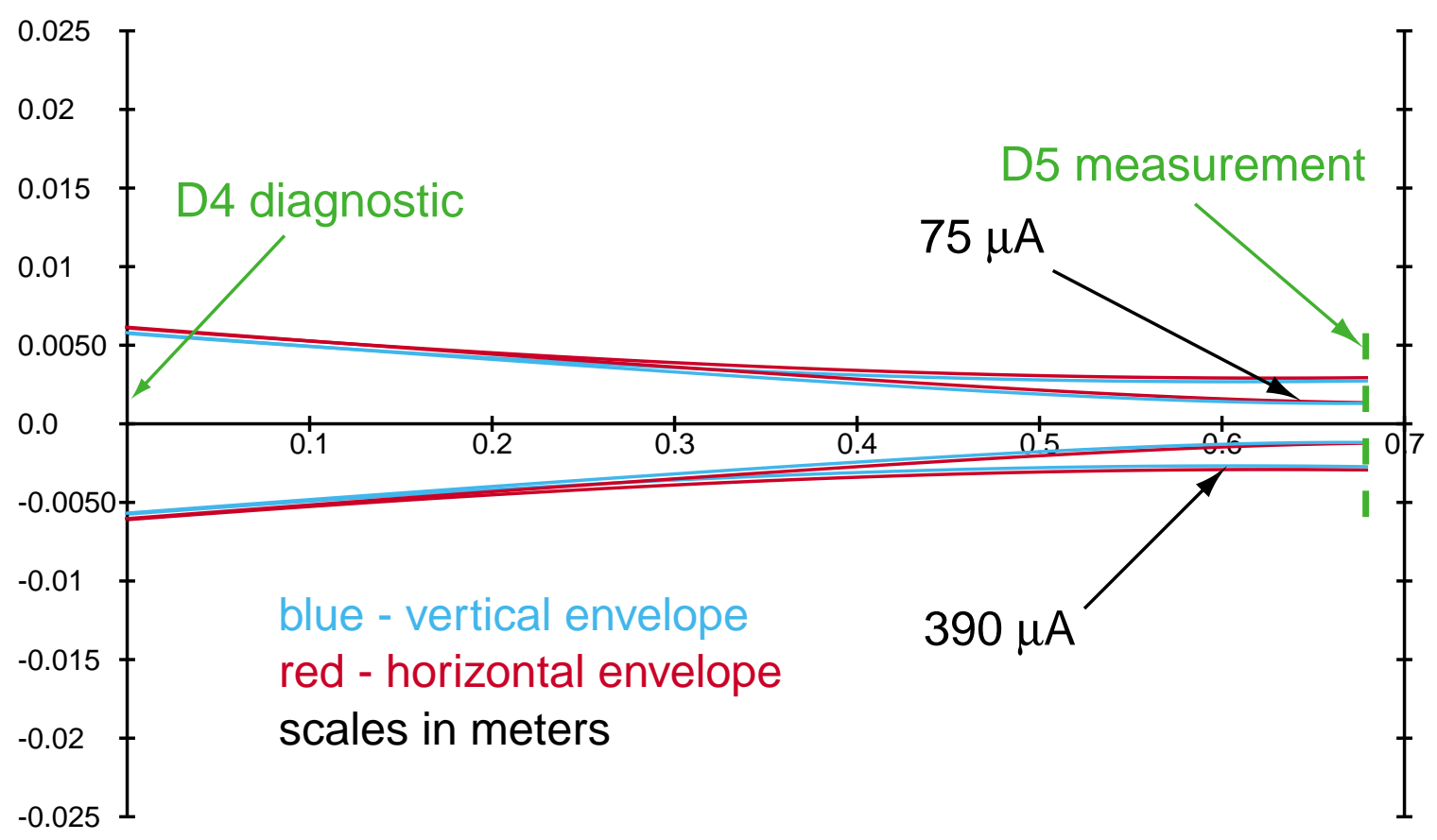

Figure 7.9: Calculated beam envelopes between D4 and the focus for both the neutralized and un-neutralized cases. 


\subsection{Comparison with the LSP Model}

From the data, it appears that the changes in the measured characteristics of the focal spot are due to the acquisition of cold $(\sim 0.2 \mathrm{eV})$ electrons from the filament. To test this conclusion, the portion of the experiment from the location of the filament at D4 to the focus is simulated using the particle in cell code LSP. This code is written to study the interaction of a heavy ion beam with the wide range of gas and plasma densities that could possibly occur in the target chamber. (Hughes, 1999, Welch, 2000). For the purposes of modeling the introduction of electrons into the focus, the code is run in a 2D, axisymmetric, electrostatic mode. The source of the neutralizing electrons in the simulation is space charge limited emission from the beam entrance plane within a radius of $12 \mathrm{~mm}$ (twice the beam radius). In order to model the filament's ample supply of electrons, the field emission threshold was set to $0.01 \mathrm{~V} / \mathrm{cm}$, much lower than the self fields within the beam (Rose, 2000, Welch, 2000).

The simulation region is $120 \mathrm{~cm}$ in length and is surrounded by a conducting boundary at $3 \mathrm{~cm}$, a factor of 5 larger than the maximum beam radius. An axisymmetric current density profile with radial variation based on the cross-slit measurement at D4 is used at $z=0$. At the entrance plane, the ions are given the transverse velocity necessary to focus them at the location of the D5 measurements. Superposed on this is a random velocity contribution equivalent to a temperature of $0.18 \mathrm{eV}$, or $9 \pi \mathrm{mm} \mathrm{mrad}$, the measured emittance. The beam current starts from zero, and is allowed to rise to $400 \mu \mathrm{A}$ in $200 \mathrm{~ns}$.

The results of the model are presented in Figure 7.10. To more accurately compare the output of the simulation with the experimental results, the charge density 
profile is collapsed onto one transverse dimension as would be done with the diagnostic slit cup. These projections are plotted on the same axes as the experimental data for the various measurements described in the previous section. The amplitudes of the individual curves are normalized by their areas, and the centroids of the experimental data are shifted to enhance the comparison. The extraordinary agreement between the curves suggests that the injection of cold electrons at the filament location is in fact responsible for the reduction in focal spot size.
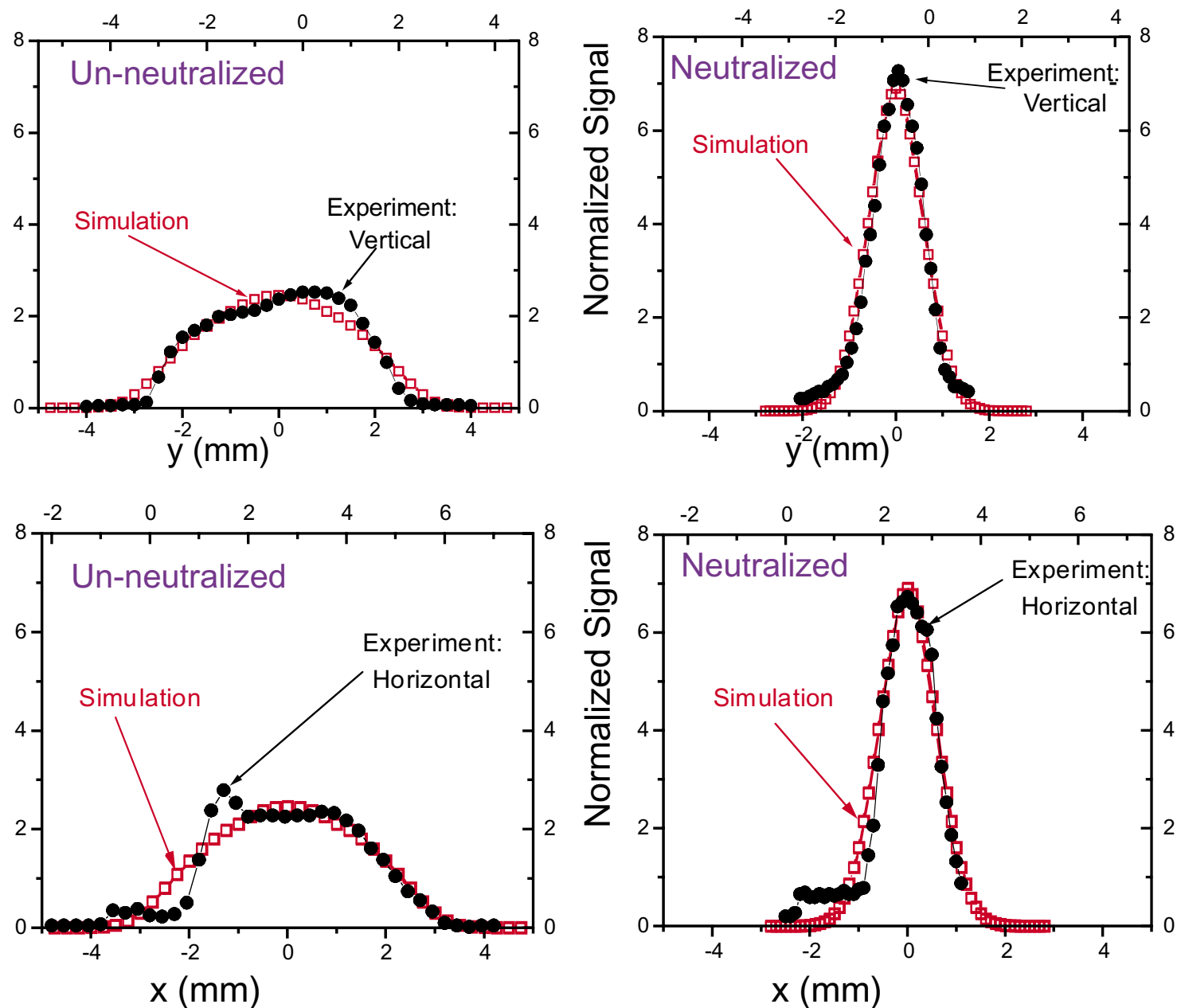

Figure 7.10 : The neutralized beam from the LSP run is plotted as it would be seen in a single slit scan. This data is compared to the measured spot. 


\section{Error Analysis and Conclusions}

\subsection{Linearity and Reproducibility of the Slit-cup Measurements}

The shallow slit and Faraday cup paddle described in Section 3.4 and depicted in Figure 3.8 is used to collect the signal in the majority of the measurements made. For the phase space measurements, two are used in series, and because the slits are very narrow the amount of ion current collected is as small as $50 \mathrm{nA}$ in some cases. Considering the circuit in Figure 3.8, a gain of 2000 is needed to acquire a $5 \mathrm{mV}$ signal from the ions collimated by the two slit combination. Therefore, in addition to the gain of the amplifier, this diagnostic relies on the enhancement of the ion signal by secondary electrons. The grid and collector are operated in a "reverse biased" configuration, with $V_{\text {collector }}$ negative and $V_{\text {grid }}$ positive, to attract the electrons away from the collector surface. For $\mathrm{Cs}^{+}$ions at several hundred keV normally incident on a stainless steel collector, there are $\sim 20$ secondary electrons produced that can be biased away from the collector surface, amplifying the ion signal from the incident ions by the same factor. The geometry of the slit, grid, and collector shown in Figure 3.8 serves to significantly reduce the effect of the dependence of the secondary electron yields on the ion angle of incidence.

An accurate measurement of beam current or phase space density using the slitcup requires that this secondary electron signal be linearly proportional to the incident ion current. To check this, measurements can be made at a location where the secondary electron gain is not necessary to resolve the signal, and the paddle biased "forward" as is done in the deep Faraday cup. This returns the secondary electrons to the collector surface so that only the ion current is measured. Such a check is performed at D5 where 
the beam is focused and the current density is at a maximum. The slit-cup mounted to the 3-axis probe is used for two identical measurements: one using the secondary electrons to amplify the signal, and the other measuring only the ion current.

The two measurements are compared in Figure 8.1. The signal from the ion current measurement is multiplied by a factor of 24.5 to normalize the amplitudes of the profiles, representing the effective secondary electron coefficient for this situation. The second moments of the to the distributions agree to within $3.9 \%$, and the widths of the Gaussian fits agree to within $1.2 \%$.

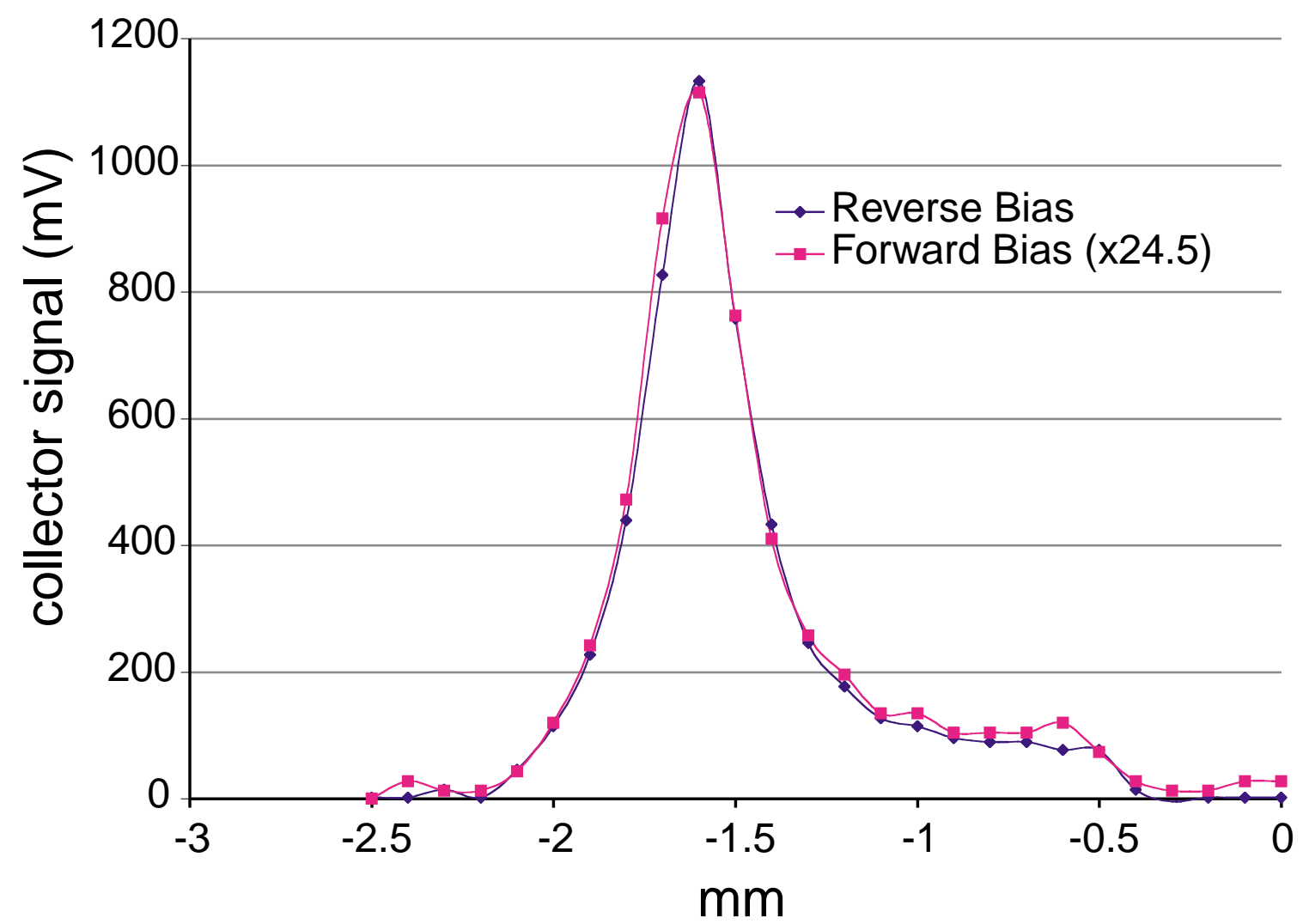

Figure 8.1: Comparisons of a profile measurement made using the secondary electron signal (reverse bias) with the ion signal (forward bias). The ion signal has been scaled by a factor of 24.5.

As described in Chapter 3, measurements made with the slit diagnostics rely on the pulse-to-pulse repeatability of the Marx generator to produce an identical beam pulse 
each time the slits are repositioned. The dynamics of a single beam pulse can then be reconstructed from individual pulse measurements performed at discrete locations in phase space. To assess the degree of repeatability, two phase space measurements are performed in succession with the same set of diagnostic probes keeping all experimental parameters constant. The second moments and the emittances of the distributions are compared, and all agree to within $0.25 \%$. This fraction represents the degree of error in the measurements introduced by fluctuations of the pulse generator, folded in with any errors associated with the accuracy of the slit paddle positioning.

\subsection{Errors Associated with Two Slit Emittance Scans}

There are two principle sources of error associated with the two slit emittance measurements. These errors arise in the determination of the rms width of the transverse velocity distribution in phase space. The data is obtained as the second slit scans through the "slice" of beam intercepted by the first slit, as depicted in Figure 3.9. One source of error inherent in this technique is a broadening of the distribution due to the finite width of the slits. The other error arises from the dynamics of the slice as it travels to the second slit and undergoes expansion due to its self fields. Both effects result in an artificially larger value for the measured emittance.

The effect of a finite slit width on an inherently Gaussian transverse velocity distribution can be estimated with a convolution integral. The slit widths and the distance between slits are known, and an angle representing the intrinsic velocity spread of the incident beam is assumed. The contributions to the signal at the second slit due to an array of points across the first slit are summed. This is shown graphically in Figure 8.2; the contributions of the beam from the red, yellow, and blue points in the first slit are 
calculated at the second slit and added. The second slit is then moved one step and the process repeated.

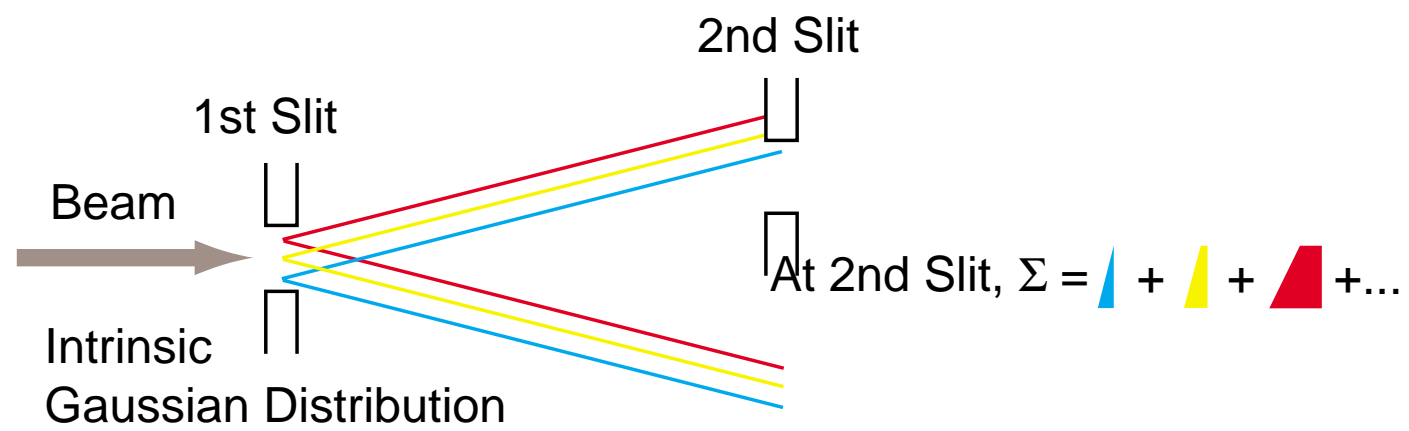

Figure 8.2: Illustration of a calculation of the expected measured velocity distribution from an intrinsic Gaussian distribution incident on a pair of slits of finite width.

An rms width of the resulting distribution can be calculated and compared with the angle assumed for the velocity spread of the incident beam. A larger slit width will result in a larger error in the measurement, but a longer drift distance helps the second slit better resolve the angles and reduces the error somewhat. The error will be fractionally larger for a smaller intrinsic transverse velocity spread. For each of the diagnostics stations used to make the emittance measurements, the slits are $50 \mu \mathrm{m}$ wide, and are separated by $15.2 \mathrm{~cm}$. At D1, where the smallest emittance was recorded for the $95 \mu \mathrm{A}$ beam, the error is estimated to be $3.4 \%$. For the larger emittance associated with the 400 $\mu \mathrm{A}$ beam, the error is only $0.3 \%$.

The effect of self fields on the two slit emittance measurement can be estimated by considering the envelope equation for a sheet beam. First, we consider an ion at the edge of such a beam travelling in the $z$ direction, with density $n$, thickness $x_{0}$, and infinite dimension in $y$. Such a beam would have a surface charge density $\sigma$, and a self field $E_{x}=\frac{\sigma}{2 \varepsilon_{0}}$. The non-relativistic equation of motion for the edge ion is 
$\ddot{x}=\frac{e}{m} \frac{\sigma}{2 \varepsilon_{0}}=\frac{e}{m} \frac{e n 2 x_{0}}{2 \varepsilon_{0}}$

We can define a sheet current density, $J_{s}=2 e n v_{z} x_{0}(\mathrm{~A} / \mathrm{m})$, and change the time

derivative to a derivative in $z$ :

$x^{\prime \prime}=\frac{e J_{s}}{2 \varepsilon_{0} m(\beta c)^{3}}$

This expression is similar to Equation 2-5, without the external focusing term. We can

then define a sheet beam perveance $K_{s}=\frac{e J_{s}}{2 \varepsilon_{0} m(\beta c)^{3}}$, and immediately write down a

sheet beam envelope equation for the beam envelope $a$ :

$a^{\prime \prime}=\frac{\varepsilon^{2}}{a^{3}}+K_{s}$

This sheet perveance is no longer dimensionless, but the perveance term in

Equation 8-3 does not depend on the beam envelope. With an initial slit beam width $a_{0}$ and envelope angle $a_{0}$, the equation can be readily integrated:

$a(z)=\sqrt{a_{0}^{2}+2 a_{0} a_{0}^{\prime}+\left(\frac{\varepsilon^{2}}{a_{0}^{2}}+a_{0}^{\prime 2}\right) z^{2}}+K_{s} \frac{z^{2}}{2}$

For the purposes of this calculation, we can consider a slit beam produced near the center of the incident beam, so that $a_{0}{ }^{\prime} \sim 0$. Additionally, the emittance scales linearly with the dimensions in phase space, so the ratio of the sheet beam emittance to the width is equal to that of the full beam emittance to its radius. The first term in Equation 8-4, the contribution due to emittance, can then be written as

$a(d)_{\text {emit }}=\sqrt{\frac{s^{2}}{4}+\frac{\varepsilon_{\text {beam }}^{2} d^{2}}{r_{\text {beam }}^{2}}}$

where $s$ is the full width of the slit, and $d$ is the distance between slits.

The second term in Equation 8-4 represents the spreading of the sheet beam due space charge. Using the definition of the sheet beam current density $J_{s}$ and perveance 
$K_{s}$, along with the beam perveance $Q$ defined in Equation 2-12, we can write the self field contribution in terms of known quantities:

$a(d)_{s c}=Q \frac{s d^{2}}{2 r_{\text {beam }}^{2}}$

It is important to point out that the slice of beam emerging from the diagnostic slit has a finite extent in $y$. This will result in a slight reduction in $J_{s}$ by the time the ions reach the second slit, so that Equation 8-6 represents an upper limit.

The ratio of Equation 8-6 to Equation 8-5 represents the contribution of space charge to the size of the distribution measured at the second slit. For the emittance measurements performed in the experiment, $s$ and $d$ are constant, and the variation in $r_{\text {beam }}$ is small. Therefore, it is the perveance and the emittance that will determine the degree to which the space charge of the sheet beam will affect the measurement. For the $95 \mu \mathrm{A}$ beam, using the lowest measured emittance of $2.7 \pi \mathrm{mm}$ mrad, the fraction is $0.17 \%$. For the higher current, using $9 \pi \mathrm{mm} \mathrm{mrad}$, the fraction is still only $0.31 \%$. These calculations show that the effect of the slit beam self field on these emittance measurements is negligible.

\subsection{Error Associated with the Drift Tube Bias}

The measurement of fractional beam neutralization with the capacitive drift tube is described in Section 7.2. Here it is mentioned that a small negative bias of $\sim 11 \mathrm{~V}$ is placed on the drift tube to prevent the neutralized beam measurement from being affected by stray electrons colliding with the tube. A rough calculation shows that due to the small transverse space charge potential of the beam, even these small bias levels could have an effect on the ability of the electrons from the filament to stay with the beam as it 
enters the tube. This would increase the net charge passing through the tube, and would result in a reduction in the observed fractional neutralization.

To test for this possibility, a series of measurements of the neutralized focus profile are made with the beam passing through the drift tube. At first the tube is left unbiased, then the magnitude of the bias potential is increased for each measurement up to $-100 \mathrm{~V}$, with the results plotted in Figure 8.3. The spot radii plotted represent twice the rms of the measured distribution. The measurements for the $0 \mathrm{~V},-11 \mathrm{~V}$, and $-20 \mathrm{~V}$ case agree with the measurement in the absence of the drift tube. It is not until the bias is raised to $-40 \mathrm{~V}$ that a measurable difference occurs in the focal spot. These results indicate that the bias level used during the drift tube measurement has no discernable effect on the fractional neutralization.

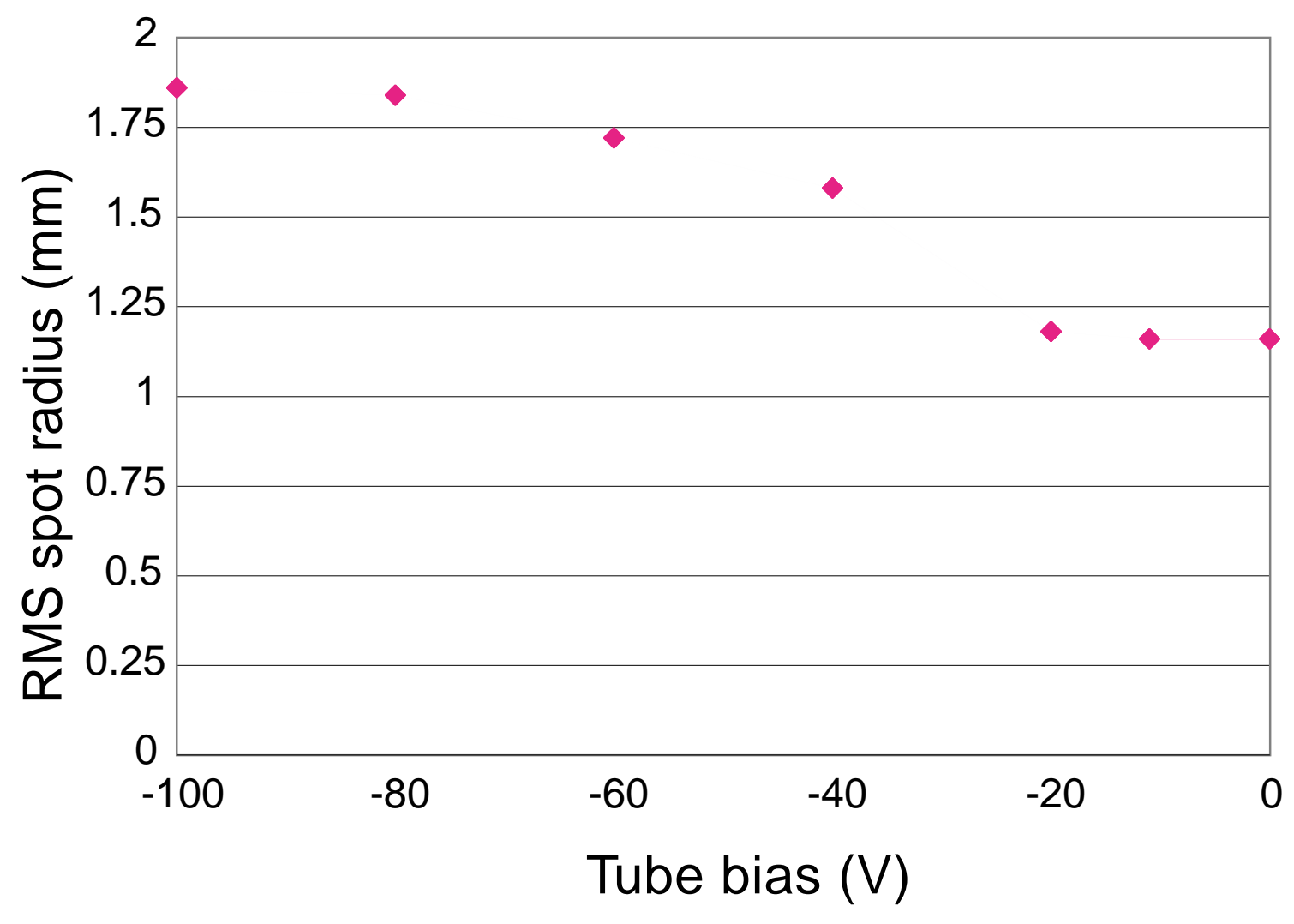

Figure 8.3: Spot size of the neutralized beam passing through the biased drift tube is plotted as a function of the negative bias voltage. 


\subsection{Conclusions}

The experiment described in this work is the first properly scaled test of a final focus design that is a potential candidate for a fusion driver. It is an accurate reproduction of the transverse beam dynamics that would exist in the full scale power plant envisioned in the HIBALL-II report. Rather than a simplified stationary distribution, the experimental beam includes the phase space distortions and nonuniformities that develop as the beam propagates through the system. Comparisons with the particle-in-cell model demonstrate that the simulation is beginning to capture some of these real effects. However, the important conclusion remains that in spite of the effects that were not present in simulations, the design focal spot criteria were met. Additionally, the study of the chromatic aberrations shows that the focusing efficiency for an energy shift of $\pm 1 \%$ is close to what was modeled by the design report. The experimental beam is less sensitive for a $\pm 2 \%$ shift than had been predicted by the report.

As was mentioned in the introduction, the probability is high that there will be some degree of neutralization from a variety of electron sources. A judicious choice of current for the experiment made possible a study of electron neutralization of the focus. Without neutralization, the $400 \mu \mathrm{A}$ beam is manipulated by the magnet system to achieve the desired convergence, and results in a focal spot size and shape dominated by the beam perveance. Despite the very weak space charge potential, the dramatic change in the nature of the focus is proof that the focusing can be improved through neutralization to near the theoretical limit by providing a source of electrons with the appropriate velocity distribution. 
There are several possibilities for further study to which the experimental apparatus lends itself. The focused beam could be used to develop improved beam spot imaging diagnostics, possibly using scintillating material and a CCD camera. This would create a more precise picture of the two dimensional energy deposition that would occur on the radiator of a target. Additionally, a more detailed survey of the performance of the iridium contact ionizer source can be made. In particular, it would be useful to measure and compare the neutral emission from the iridium with that of a tungsten source at the same temperature, and also dope the source with potassium carbonate in order to measure the lifetime for $\mathrm{K}^{+}$emission.

By sending a beam incorporating typical perturbations in phase space and current density distribution through a lattice containing focusing field imperfections of the appropriate magnitude, the experiment strives to validate the "main line" approach of ballistic focusing using magnetic quadrupoles. No beam dynamics phenomena were uncovered that would render this approach unfeasible for a fusion driver. With the understanding of the transverse beam dynamics, this experiment sets the stage for future experimental efforts incorporating longitudinal compression.

In light of the neutralized focus results presented in this work, the question of how sources of electrons in the chamber can be beneficial for the focus merits further study. Because one of these sources is likely to be due to stripping of the beam ions, the problem is further complicated by the change in charge state of the beam, and the uncertainties in the atomic cross sections associated with collisions with the background gas. Some computational work has been done on this problem (Barboza, 1996, and Callahan, 1996), and more efforts are currently underway (Welch, 2000, Sharp, 2000, 
Armel, 2000). However, due to the complex nature of the issue and the requirements of the target, experiments involving higher beam energies and potentials and more realistic background pressures are needed to compliment and validate the models before a driver design can be finalized. 


\section{References}

Armel, M. S. “Atomic Processes for Heavy Ion Inertial Fusion.” Ph.D. diss., University of California, Berkeley, 2000.

Bangerter, R. O., and R. M. Bock. "Heavy Ion Drivers.” Energy From Inertial Fusion. Ed. W. J. Hogan. Vienna, International Atomic Energy Agency, 1995.

Bongardt, K., and R. W. Müller. "Linac Power Efficiency." The HIDIF Study: Report of the Europe Study Group on Heavy Ion Driven Inertial Fusion for the period 19951998, GSI-90-06, Darmstadt, August 1998. 69-72.

Brady, V.O., A. Faltens, D. Keefe, E. P. Lee, and J. Hovingh. "Heavy Ion Fusion System Assesment: Final Focus and Transport Model.” LBL-23040, Lawrence Berkeley National Laboratory, July 1987. 7-9.

Brewer, G. R., M. R. Currie, and R. C. Knechtli. "Ionic and Plasma Propulsion for Space Vehicles." Proceedings of the IRE, December (1961):1789-1821.

Callahan, D. A. "Chamber propagation physics for heavy ion fusion." Fusion Engineering and Design, 32-33(1996): 441-452.

Callahan-Miller, et al. "Progress in heavy ion fusion targets." Proceedings of the 13th International Symposium on Heavy Ion Inertial Fusion, March 13-17, 2000. To be published in Nuclear Instruments and Methods in Physics Research A.

Celata, C. M. et al. "Transverse combining of four beams in MBE-4." Fusion Engineering and Design, 32-33(1996): 219-224.

Chan, Chun Fai et al. "Dynamics of Skew Beams and the Projectional Emittance." Nuclear Instruments and Methods in Physics Research A, 306 (1991): 112-122. 
De Hoon, M. J. L. et al. "Simulation of the LBNL Scaled Final Focus Experiment." Proceedings of the 13th International Symposium on Heavy Ion Inertial Fusion, March 13-17, 2000. To be published in Nuclear Instruments and Methods in Physics Research A.

De Hoon, M. J. L., W. Fawley, and J. Novotny. "Java Envelope: A code for beam envelope modeling." Computer program written in the Java programming language, based the Basic code "Rematch" written by K. Hahn, and converted to Fortran by C. M. Celata.

Feldbacher, Rainer. “The Alternate Energy Physics Program Barnbook DATLIB: Nuclear Reaction Cross Sections and Reactivity Parameter Library and Files", Technical Report INDC(AUS)-12/G, IAEA Nuclear Data Section, Wagramerstrasse 5, A-1400, Vienna, Austria, Oct. 1987.

Forrester, Theodore A., Large Ion Beams, New York: Wiley, 1988.

Grote, D. P., et al. "New developments in WARP: Progress toward end-to-end simulation." Nuclear Instruments and Methods in Physics Research A, 415 (1998): 428-432.

Herrmannsfeldt, W. B. "Electron Trapping in High-Current Ion Beam Pipes.” Proceedings of the 13th International Symposium on Heavy Ion Inertial Fusion, March 13-17, 2000. To be published in Nuclear Instruments and Methods in Physics Research A.

Hogan, W. J. et al. “Energy From Inertial Fusion.” Physics Today, 45,9 (1992): 42-50.

Hovingh, J. et al. "Heavy-Ion Linear Induction Accelerators as Drivers for Inertial Fusion Power Plants.” Fusion Technology, 13 (1988): 255-278. 
Humphries, Stan, Charged Particle Beams, New York: Wiley, 1990.

Kapchinskij, I. M. and V. V. Vladimirskij. "Limitations of Proton Beam Current in a Strong Focusing Linear Accelerator Associated with the Beam Space Charge." Proceedings of the 1959 International Conference on High Energy Accelerators. Geneva, Switzerland: CERN, 1959. 274 ff.

Kwan, J. W. "Ion sources for heavy ion fusion induction linacs." Nuclear Instruments and Methods in Physics Research A, 415 (1998): 268-273.

Lampel, M, and M. G. Tiefenback. "An applied voltage to eliminate current transients in a one-dimensional diode.” Applied Physics Letters, 43(1) (1983): 57-58.

Latkowski, J., and W. Meier. "Improved final focus shielding designs for modern heavyion fusion power plant designs." Proceedings of the 13th International Symposium on Heavy Ion Inertial Fusion, March 13-17, 2000. To be published in Nuclear Instruments and Methods in Physics Research A.

Olson, C. L. et al. "Microcharge neutralization transport experiments and simulations for ion-driven inertial confinement fusion." Fusion Engineering and Design 3233(1996): 485-491.

Pierce, J.R. Theory and Design of Electron Beams, Princeton: Van Nostrand, 1949.

R.W. Moir, et al. "HYLIFE-II Progress Report", UCID-21816, Lawrence Livermore National Laboratory, 1991.

Reiser, Martin. Theory and Design of Charged Particle Beams, New York: Wiley, 1994. Rose, D. V., D. R. Welch, and S. A. MacLaren. "Beam Neutralization in the Scaled Final Focus Experiment.” MRC/ABQ-R-1995, Mission Research Corporation, Albuquerque, NM, June 2000. 
Seidl, P. A. et al. "Progress on the scaled beam-combining experiment at LBNL." Nuclear Instruments and Methods in Physics Research A, 415 (1998): 243-248.

Sharp, W. M. et al. "Improved modeling of chamber transport for heavy ion fusion." Proceedings of the 13th International Symposium on Heavy Ion Inertial Fusion, March 13-17, 2000. To be published in Nuclear Instruments and Methods in Physics Research A.

Taylor, John B. and Irving Langmuir, Physical Review 44, 6 (1933): 423.

Tiefenback, M. G. "Space-Charge Limits on the Transport of Ion Beams in a Long Alternating Gradient System.” Ph.D. diss., University of California, Berkeley, 1986.

Welch, D. R. and C. L. Olson. "Self-pinched transport for ion-driven inertial confinement fusion." Fusion Engineering and Design 32-33(1996): 477-483.

Welch, D.R., D. V. Rose, B. V. Oliver, and R. E. Clark, "Simulations of Chamber Transport." Proceedings of the 13th International Symposium on Heavy Ion Inertial Fusion, March 13-17, 2000. To be published in Nuclear Instruments and Methods in Physics Research A.

Wollnik, H. et al. "HIBALL-II: An Improved Conceptual Heavy Ion Beam Driven Fusion Reactor Study”, KfK-3840, FPA-84-4, UWFDM-625, Karlsruhe, July 1985. 57-71.

Yu, S. et al. "Plasma-channel-based reactor and final transport." Nuclear Instruments and Methods in Physics Research A, 415 (1998): 174-181. 


\section{Appendix A: A condensed history of final focus studies}

The problem of final focus has been a principal issue fusion for heavy ion fusion driver concepts since the first serious consideration in 1976. This appendix attempts to summarize key steps in the evolution of final focus designs from that time until the present in hope of placing the considerations of the current work in historical context. As one would expect, the steady improvement of computing power has had an impact on the development of these designs, evidenced by the increasing level of detail of the beam dynamics in each subsequent concept. The author wishes to acknowledge the efforts of Dr. David Judd in distilling from the literature a fairly exhaustive list of works pertaining to heavy ion final focus.

Beginning with the ERDA Summer Study of Heavy Ions for Inertial Fusion held in Berkeley in 1976, a working group was tasked with exploring options for delivering the beam from the accelerator to the target. A system was proposed consisting of two unseparated quadrupoles of equal gradients, minimizing the number of free parameters in the design. These focusing elements are treated with transfer matrices without aberration, and the effects of space charge are not included (Garren, 1976). The next design was more complex, consisting of six quadrupoles arranged in two triplets (Colton, 1978). It also incorporated sextupoles and octupoles, elements already in use in particle accelerators for high energy physics for the correction of chromatic and geometric aberrations, respectively. Chromatic aberrations are described in section 1.4, and geometric aberrations arise due to deviations from paraxial ray optics if the beam is focused too abruptly. For this report, computer codes were used to determine the 
placement and strength of these additional elements, and the results were checked with a ray tracing program. The effects of space charge are not included. In the same proceedings, a limit on the final convergence angle of the beam to the focus is proposed to minimize the effect of the third order geometric aberrations on the focal spot size; using this limit with practical driver parameters, the maximum convergence angle is 2030 milliradians (Neuffer, 1978).

To include the effects of space charge as a linear force, a focusing system was designed using the beam envelope equation, derived in Chapter 2. This beam line consisted of six quadrupoles, with no other types of elements included (Garren, 1979). It explored the possibility of a system without sextupole corrections that would be less sensitive to momentum spread (width of the longitudinal velocity distribution). The beam envelopes were tracked through the system, including off-momentum trajectories, with mixed results. The report concludes that it is desirable to create beam envelopes that are symmetrical on average, and to avoid small waists at intermediate points in the final focus. A subsequent report investigated these conclusions in a design that consisted of three quadrupole triplets (Garren, Krafft, and Haber, 1981). This work made use of the increasing availability of computing power with a code that placed the elements using matrix methods, then calculated the gradients necessary to focus a beam with space charge by integrating the envelope equation. Finally, the focusing lattice was checked using a particle simulation that ran several thousand macroparticles through the elements. Not only did this perform well for the momentum spread, but the simulation demonstrated an acceptance of non-uniform transverse current distributions. 
In chronological order, the next report is that of the HIBALL-II final focus subsystem upon which the experiment discussed in this thesis is based. It was designed using an ion optics code to set the focusing gradients and minimize geometric aberrations. This code was used to study the effects of chromatic aberrations due to both a momentum spread, and an overall head to tail velocity difference, or tilt, for longitudinal beam compression (Wollnik, 1984). It is important to note that HIBALL-II final focus was chosen as a realistic model for the subsequent Heavy Ion Fusion System Assessment which strove to provide a comprehensive costing code for a power plant (Brady, 1987).

In the 1990's, improvements in numerical simulation capability made possible the fine tuning of final focus designs to control the effects of various aberrations, and even play them against each other to minimize the focal spot size. First, a study was performed using the non-uniform line charge density profiles that were being considered to provide pre-heating of the target prior to the arrival of the peak pulse (Ho, Crandall, Haber, 1992). Close attention was paid to symmetry of a five quadrupole system with respect to the longitudinal midpoint in order to suppress the effect of the velocity tilt used to help shape the current pulse. A code that integrated the envelope equation was used to successfully preserve the desired line charge density profile and minimize the chromatic effects by locating the point where the velocity tilt is zero near the midpoint of a symmetric system.

Some fusion target designs require a main pulse and a preheating pulse that could differ in magnitude by a factor of five be delivered to the target. A subsequent report that considered a four quadrupole system addressed this issue by exploiting the existence of a 
minimum focal spot radius as function of current (Ho, Crandall, Haber, 1994). For a careful choice of beam parameters, the space charge force at the focus could be matched to the pressure gradient due the emittance at a current that was between the preheat and peak values, resulting in minimum variation of the spot size over the duration of the pulse. Again, an envelope code was used to design the system and determine the beam parameters, and a two-and-one-half dimensional particle in cell code model was used to include the self-consistent evolution of the beam distribution and a non-zero momentum spread.

Finally, the most recent work on a final focus system was done for a study that would use beam bunches of three different ion species from an RF accelerator to drive the target. This design consisted of six quadrupoles plus 2 octupoles that would help compensate for geometric aberrations from a $30 \mathrm{mrad}$ convergence angle (Spiller, 1998). Chromatic aberrations arising from bunch compression techniques employed between the accelerator and the focus were viewed to be the most serious challenge, but the variation of the line charge density along the length of the bunch was also considerable. Again, a clever choice of beam parameters was made, and these two effects were allowed to compensate for one another. Both the maximum momentum spread due to compression and the peak current were to occur at the middle of the pulse. The design was therefore tuned for the minimum spot size to occur at the current maximum, resulting in the least variation in spot size at the target. 


\subsection{Appendix A References}

Brady, V.O., A. Faltens, D. Keefe, E. P. Lee, and J. Hovingh. "Heavy Ion Fusion System Assesment: Final Focus and Transport Model.” LBL-23040, Lawrence Berkeley National Laboratory, July 1987. 7-9.

Colton, E. "Correction of Chromatic and Geometric Aberrations Using Sextupoles and Octupoles." Proceedings of the Heavy Ion Fusion Workshop Held at Argonne National Laboratory September 19-26, 1978. ANL-79-41, 1979. 365-378.

Garren, A.A. "Final Focusing of the Ion Beams of a Pellet Fusion Reactor by Quadrupole Doublets." ERDA Summer Study fo Heavy Ions for Inertial Fusion, July 19-30, 1976. Eds. R. O. Bangerter, W. B. Hermannsfeldt, D. L. Judd, and L. Smith. LBL-5543, 1976. 102-109.

Garren, A. A. "Studies of a Beam Line for Transport to a Target." Proceedings of the Heavy Ion Fusion Workshop, October 29 - November 9,1979. Ed. W. B. Herrmannsfeldt. LBL-10301, SLAC-PUB-2575, 1980. 397-402.

Garren, A. A., G. Krafft, and I. Haber, "Focusing of Heavy Ion Beams on a Fusion Target.” IEEE Transaction on Nuclear Science, NS-28,3 (1981): 2468-2470.

Ho, D. D-M., K. R. Crandall, and I. Haber. “Achromatism in Final Focusing Systems for High-Current Heavy-Ion Beams.” Particle Accelerators, 37-38 (1992): 155-160.

Ho, D. D-M., K. R. Crandall, and I. Haber. "Focusing Beams with Widely Varying Current Using Fixed Strength Quadrupoles for Heavy-Ion Inertial Fusion.” Particle Accelerators, 45 (1994): 119-134. 
Neuffer, D. "Geometric Aberrations in Final Focussing for Heavy Ion FusionProceedings of the Heavy Ion Fusion Workshop Held at Argonne National Laboratory September 19-26, 1978. ANL-79-41, 1979. 333-339.

Spiller, P., and I. Hoffman. "Optics of Final Beam Transport and Focusing for HIDIF." The HIDIF Study: Report of the Europe Study Group on Heavy Ion Driven Inertial Fusion for the period 1995-1998, GSI-90-06, Darmstadt, August 1998. $145-152$. 